\title{
ESTIMABILIDADE DE FUNÇØ̃ES PARAMÉTRICAS COM DADOS DESBALANCEADOS ATRAVÉS DO PROC GLM DO SAS: APLICAÇסES À PESQUISA AGROPECUÁRIA
}

MARCI A MONDARDO

Dissertação apresentada. à Escola Superior de Agricultura "Luiz de Queiroz", da Universidade de São Paulo, para obtenção do título de Mestre em Agronomia, Area dé Concentração: Estatistica e Experimentação Agronómica.

\footnotetext{
$P I R A C I C A B A$

Estado de São Paulo - Brasil

Jul ho - 1894
} 


\title{
ESTIMABILIDADE DE FUNÇOES PARAMÉTRICAS COM DADOS DESBALANCEADOS ATRAVÉS DO PROC GLMDO SAS: APLICAÇOES $\dot{A}$ PESQUISA AGROPECUÁRIA
}

\section{MARCI A MONDARDO}

\author{
Engenheira Agrónoma
}

Orientador: Prof. Dr. Antonio Francisco Iemma

Dissertação apresentada à
Escola Superior de Agricultura
"Luiz de Queiroz", da
Universidade de São Paulo, para
obtenção do titulo de Mestre em
Agronomia, Area de Concentração:
Estatistica = Experimentação
Agronomica.

$P I R A C I C A B A$

Estado de São Paulo - Brasil

Jul ho -1994 


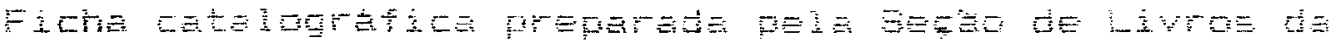

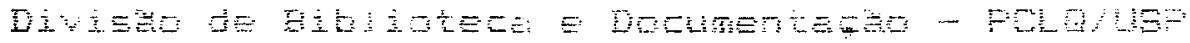

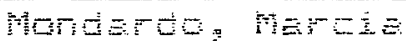

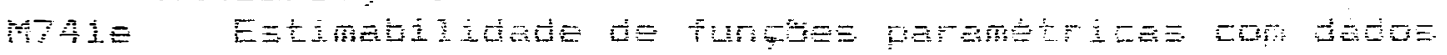

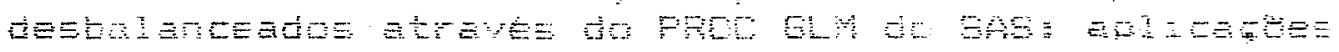

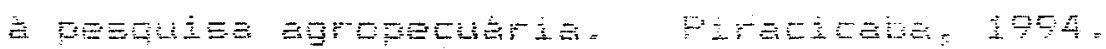
$\dot{E}=\bar{T}$

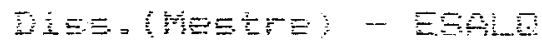

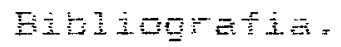

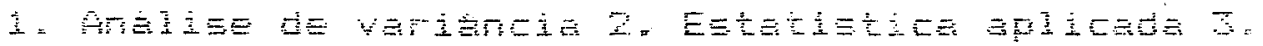
HOAE Wor dE ADr 


\section{ESTIMABILIDADE DE FUNÇÕES PARAMÉTRICAS COM DADOS DESBALANCEADOS ATRAVÉS DO PROC GLM DO SAS: APLICAÇÕES À PESQUISA AGROPECUÁRIA}

\section{MARCI A MONDARDO}

Aprovado em: 29.08. 94

Comissão Julgadora:

Prof. Dr. Antonio Francisco Iemma

ESALQ USP

Prof ${ }^{a}$. Dr ${ }^{a}$. Maria Cristina Stolf Nogueira

Prof. Dr. Lúcio Benedito Kroll

Prof. Dr. ANTONfO FRAPlat SCO IEMMA

orientapo

ESALQ USP

B $\mathrm{B}$ UNESP

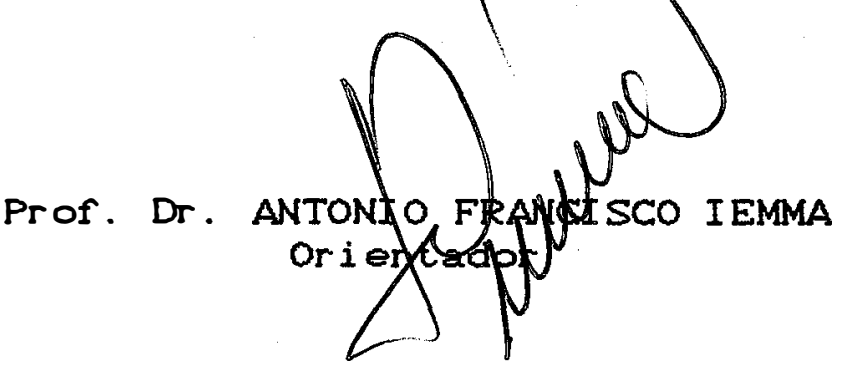


Ao Mário

A Monike

Aos meus pais,

Eucl ides e Nel ma

Dedico 
Ao Professor Antonio Francisco Iemma, pela orientação e apoio.

A Empresa de Pesquisa Agropecuária e Difusão de Tecnologia de Santa Catarina - EPAGRI.

A Empresa Brasileira de Pesquisa Agropecuária EMBRAPA, pela concessão de bolsa de Mestrado.

A todos os professores, funcionários e colegas da Escola Superior de Agricultura "Luiz de Queiroz".

Aos colegas da Estação Experimental de Caçador EPAGRI . 


\section{SUXARIO}

Página

LISTA DE TABELAS ................. xi

RESUMO $\ldots \ldots \ldots \ldots \ldots \ldots \ldots \ldots \ldots \ldots \ldots \ldots \ldots \ldots \ldots$

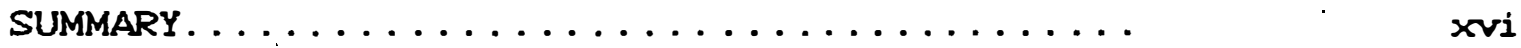

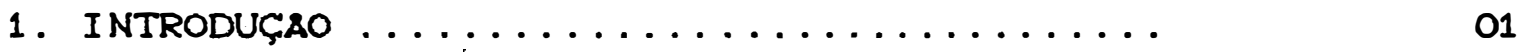

2. REVISAO DE LITERATURA .............. 03

3. METODOLOGIA .................. 35

3. 1. MATRI ZES I NVERSAS GENERALI ZADAS . . . . 35

3.1.1. Inversa Generalizada de Moore

Penrose ................ 36

3.1.2. Inversa Generalizada de Mínimos

Quadrados ............... 36

3.1.3. Inversa Condicional ......... 37

3.1.4. Inversa Generalizada G2 ...... 37

3. 2. ALgumas IDEI AS SOBRE MODELOS LINEARES 38

3.2.1. Modelo Super parametrizado ..... 39

3.2.1.1. EquaçÖes Normais .... 39

3.2.1.2. Análise da Variáncia. 41

3.2.1.3. Notação RC J ....... 42

3.2.2. Modelo de Médias de Caselas ... 43

3.2.2.1. Equaçชిes Normais .... 44

3.2.2.2. Análise da Variáncia. 45

3. 2.3. Modelo com Restrição

Parametrica do Tipo $\Sigma \ldots \ldots \ldots \ldots$ 
vi i .

3.2. 3.1. Equaçס̄es Normais ..... 45

3.2.3.2. Análise da Variáncia. 45

3. 3. ALGUMAS IDEI AS SOBRE ESTIMAÇAO . . . . . 46

3. 4. OS QUATRO TIPOS DE FUNÇסES ESTIMAVEIS 48

3.4.1. Forma Geral de FunçZ̋es

Estimáveis ............. 48

3. 4.2. Funçช̆es Estimáveis do Tipo I .. 48

3. 4.3. Funçర̋es Estimâveis do Tipo II. 50

3. 4. 4. Funçơes Estimáveis do Tipo III 51

3.4.5. Funçชes Estimáveis do Tipo IV. 52

3.5. HIPOTESES MAIS COMUNS SOBRE LINHAS,

COLUNAS E INTERAÇAO .............

3.5.1. Hi póteses Sobre a Interação ... 55

3.5.2. Hipóteses Mais Comuns Sobre

Efeitos de Linhas .......... 56

3.5.2.1. Hipóteses Sobre Médias

Ponderadas de Linhas 56

3.5.2.2. Hi póteses Sobre Médias

Ponderadas de Linhas

Ajustada para Colunas $\quad 57$

3.5.2.3. Hi póteses Sobre Médias

Nao Ponderadas de

Linhas ...........

58

3.5.2. 4. Hi póteses Sobre Médias

Não Ponderadas de

Linhas, Segundo as 
$v i \mathrm{i}$.

Caselas Vazias .......

3.5.3. Hipóteses Mais Comuns Sobre Efeitos de Colunas ..........

3.5. 3.1. Hi póteses Sobre Médi as Ponderadas de Col unas

3.5.3.2. Hi póteses Sobre Médias Ponderadas de Colunas Ajustadas para Linhas

3.5. 3. 3. Hi póteses Sobre Médias Não Ponderadas de Col unas ............ 61

3.5. 3. 4. Hi póteses Sobre Médi as Não Ponderadas de Colunas, Segundo as Casel as Vazias ...... 62 3.6. COMENTARIOS ADICIONAIS SOBRE AS FUNÇOES ESTIMAVEIS E HIPOTESES TIPO III $\ldots \ldots \ldots \ldots \ldots \ldots \ldots \ldots$

3.6.1. Outra Forma de Obtenção das Funçơes Esti máveis Tipo III ... 63 3.6.2. Um Algoritmo para Transformar as Hipóteses em Termos do Modelo $\Sigma$ em Hipóteses em Termos do Modelo S Quando Ocorrem Casel as Vazias ............. 
3. 8. 0 SISTEMA SAS $\ldots \ldots \ldots \ldots \ldots \ldots \ldots \ldots$

4. DISCUSSAO DE EXEMPLOS NUMERICOS. . . . . . . 69

4.1. 1ㅇ CASO: FATORI AL $2 \times 3$ COM UMA CASELA VAZIA $\ldots \ldots \ldots \ldots \ldots \ldots \ldots \ldots \ldots \ldots$

4.1.1. Modelos Lineares ............. 71

4.1.2. Obtenção da Forma Geral de

Funçđ̆es Estimáveis ......... 72

4.1.3. Obtenção das Funçð̋es Estimáveis Tipo $I \ldots \ldots \ldots \ldots \ldots \ldots$

4.1.4. Obtenção das Funçত̃es Esti máveis

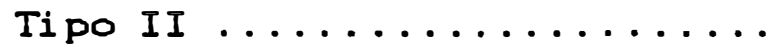

4.1.5. Obtenção das FunçZes Estimáveis Tipo III $\ldots \ldots \ldots \ldots \ldots \ldots$

4.1.6. Obtenção das Funçzes Estimâreis Tipo IV ................ 100

4.1.7. Consequéncias da Alteração da Ordem de Especificação do

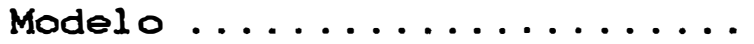

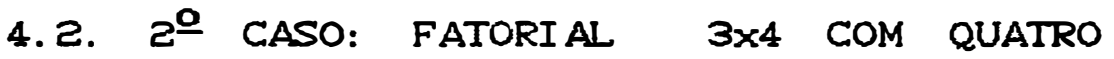

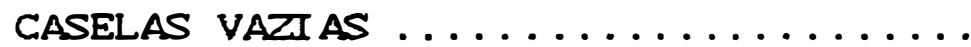

4.2.1. Modelo Li near ............

4.2.2. Obtenção da Forma Geral de

Funçỡes Estimáveis .........

4.2.3. Obtenção das Funçชes Estimâveis Tipo $\quad$ I $\quad \ldots \ldots \ldots \ldots \ldots \ldots \ldots$

4.2.4. Obtenção das Funçত̃es Estimáveis 
Tipo II ............. 126

4.2.5. Obtenção das Funçơes Estimáveis Tipo III .............. 133

4.2.6. Obtenção das FunçZ̃es Estimáveis Tipo IV ..................

4.2.7. Consequéncias da Alteração da Ordem de Especificação do Modelo ao PROC GLM .......... 150

5. CONCLUSסES ........................ 156

REFERENCI AS BIBLIOGRAFICAS ............. 159

APENDICE $\ldots \ldots \ldots \ldots \ldots \ldots \ldots \ldots \ldots \ldots \ldots \ldots \ldots \ldots \ldots$ 


\section{LISTA DE TABELAS}

Página

Tabela 1 - Hipóteses Associadas ao Modelo de Classificação Dupla ............... Og

Tabela 2 - Somas de Quadrados Tipo II em Termos da

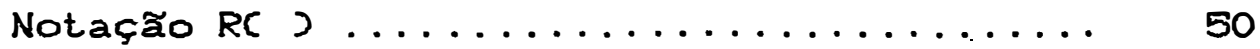

Tabela 3 - Número de Observaçôes Referentes a Cada Médi a de Casela .................. 54

Tabela 4 - Número de Observaçชes por Casela ......... 63

Tabela 5 - Coeficientes m para a Combinação Linear $f$ e

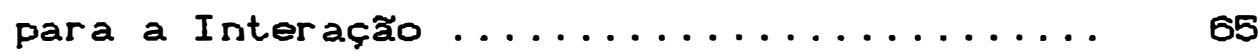

Tabela 6 - Dados em kg de Frutos/Planta ........... 70

Tabela 7 - Número de ObservaçZes por Casela Conforme

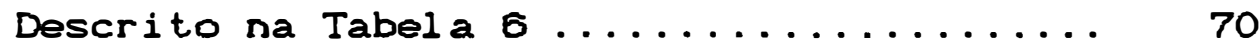

Tabela 8 - Forma Geral de Funçß̋es Estimáveis para o Modelo Fatorial com Interação Aplicado aos Dados da Tabela $6 \ldots \ldots \ldots \ldots \ldots \ldots$

Tabela 9 - Funçชes Estimáveis Tipo I para A ....... 77

Tabela 10 - Funçठ̋es Estimáveis Tipo I para B ....... 80

Tabela 11 - Funçठ̋es Estimáveis Tipo I para AxB ...... 83

Tabela 12 - Funçס̋es Estimáveis Tipo II para A ....... 86

Tabela 13 - Funçठ̋es Estimáveis Tipo II para B...... 89

Tabela 14 - Funçס̋es Esti máveis Tipo II para AxB ..... 90

Tabela 15 - Funçőes Estimáveis Tipo III para A ...... 92

Tabela 16 - Funçס̋es Estimáveis Tipo III para B ...... 94 
Tabela 17 - Coeficientes m para a Combinação Linear $f$ e para a Interação ............... 96

Tabela 18 - Funçס̃es Estimáveis Tipo IV para A...... 101

Tabela 19 - Funçס̋es Estimáveis Tipo IV para B ...... 102

Tabela 20 - Funçỡes Estimáveis Tipo I para B cordem

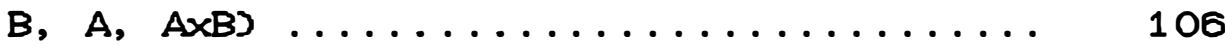

Tabela 21 - Análise de Variáncia dos Dados da Tabela

6 Através do Modelo com Dois Fatores e

Inter ação, para a Ordenação A, B, AxB ... 108

Tabela 22 - Análise de Variáncia dos Dados da Tabela

6 Através do Modelo com Dois Fatores e

Interação, para a Ordenação B, A, AxB ... 109

Tabela 23 - Número de Frutos $/$ Planta ............. 110

Tabela 24 - Número de Observaçชes por Casel a . . . . . . 111

Tabela 25 - Forma Geral de Funçס̃es Estimáveis para os Dados do Exemplo da Tabela $23 \ldots \ldots . . . . .115$

Tabela 26 - Funçס̋es Estimáveis Tipo I para A....... 118

Tabela 27 - Funçช̋es Estimáveis Tipo I para B ....... 121

Tabela 28 - Funçס̋es Estimáveis Tipo I para AxB ...... 124

Tabela 29 - Funçס̋es Estimáveis Tipo II para A ....... 128

Tabela 30 - Funçס̋es Estimáveis Tipo II para B ....... 132

Tabela 31 - Funçช̃es Estimáveis Tipo II para AxB ..... 133

Tabela 32 - Funçช̋es Estimáveis Tipo III para A ...... 134

Tabela 33 - Coeficientes m para a Combinação Linear $f$ e para a Interação ................ 137

Tabela 34 - Funçช̋es Estimáveis Tipo III para B ...... 140 
$x i \mathrm{i} i$.

Tabela 35 - Funçס̋es Estimáveis Tipo IV para A....... 145

Tabela 36 - Funçớes Estimáveis Tipo IV para B ...... 148

Tabela 37 - Funçôes Estimáveis Tipo I para B cordem

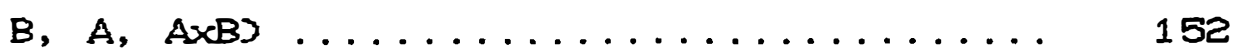

Tabela .38 - Analise de Variáncia dos Dados da Tabela 23 Através do Modelo com Dois Fatores e Interação, para a Ordenação A, B, AxB ... 154

Tabela 39 - Analise de Variáncia dos Dados da Tabela 23 Através do Modelo com Dois Fatores e Interação, para a Ordenação B, A, AxB ... 155 
xiv.

\section{ESTIMABILIDADE DE FUNÇठ̄ES PARAMÉTRICAS COM DADOS DESBALANCEADOS ATRAVÉS DO PROC GLM DO SAS: APLICAÇðES Ȧ PESQUISA AGROPECUÁRIA}

Autor a: MARCIA MONDARDO

Orientador: PROF. DR. ANTONIO FRANCISCO IEMMA

RESUMO

Este trabalho foi desenvol vido com o objetivo de alertar os pesquisadores para a complexidade da análise de dados desbalanceados com caselas vazias e orientálos na escolha do método de análise mais adequado.

- programa computacional entitulado "Statistical Analysis Sistem" (SAS) atraves de seu "General Linear Models Procedure" CPROC GLM utiliza a teoria de funçđ̄es estimáveis para prover quatro tipos de somas de quadrados para dados desbal anceados.

Atraves de dois exemplos com diferentes padróes de caselas vazias, se mostrou passo a passo, a construção das funçơes estimáveis que geram os quatro tipos de somas de quadrados fornecidas pelo SAS e que são diferentes combinaçбes lineares dos parametros do modelo.

Al em da construção foram feitas tentativas no 
sentido de interpretar 0 que as somas de quadrados provenientes destas funçశes, não rar - muito complexas, pois não envolvem somente os parametros de interesse, estão testando.

Ao pesquisador, de posse destas informaçôes, cabe escolher quais destas funçôes estimávels săo adequadas para representar a hipotese de seu interesse, poderido mesmo concluir que nenhuma delas $\epsilon$ adequada ao seu conjunto de dados. 
xvi .

ESTIMABILITY OF PARAMETRICS FUNCTIONS WHIT UNBALANCED DATA THROUGH THE PROC GLM OF SAS: APLICATION TO AGRICULTURAL RESEARCH

Author: MARCI A MONDARDO

Adviser: PROF. DR. ANTONIO FRANCI SCO IEMMA

\section{SUMMARY}

This study was developed with the purpose to alert researchers about the complexity of analysis of unbalanced data with missing cells, as well as to give them some orientations to choose the more adequate method.

The program named "Statistical Analysis System" (SAS), with its "General Models Procedure" CPROC GLMD, uses the theory of estimated functions in order to provide four types of sum of squares for unbal anced data.

Using two samples with different patterns of missing cells, it was shown, step by step, the construction of estimated functions that generate the four types of sum of squares which are given by SAS. They are different linear combinations of the parameters of the model. It was also tried to interpret what the sum of squares originated from this functions are testing. 
xvii-

The researcher needs to choose which of these functions are adequate in order to represent the hypothesis of his interest. It is possible to him to conclude that none of the functions are adequate to the data to be analyzed. 


\section{INTRODUCÃ̃}

Quando se trata de dados balanceados, as técnicas de análise de variáncia são amplamente conhecidas e di vulgadas. Para dados desbalanceados, no entanto, isto pode não ocorrer. E comum tratarem-se tais dados usando-se extensơes das técnicas conhecidas para dados balanceados ou estimação de parcel as perdidas.

Quando os dados são desbal anceados, surge a

necessidade de se conhecer que hipóteses estão sendo testadas e se estas são de interesse para o pesquisador. Al ém disso, podem ocorrer caselas vazias, dificultando ainda mais a interpretação das verdadeiras hipóteses.

A bibliografia consultada apresenta algumas regras práticas para construção de hipóteses mais comuns bem como métodos para obtenção das somas de quadrados para testarem-se as referidas hipóteses.

- pacote computacional entitulado Statistical Analysis Sistem (SAS), um dos mais importantes pacotes estatisticos da atualidade, fornece quatro tipos de funçбes estimáveis através de seu procedimento para análise de 
modelos lineares CPROC GLMD. Com base nessas funçס̄es estimáveis, são construldas as hipóteses associadas aos tipos I, II, III e IV de somas de quadrados, excelentes na análise de dados desbalanceados, mas praticamente desconhecidas dos pesquisadores de modo geral.

Com o intuito de orientar e esclarecer os pesquisadores da área agronómica, usuários do PROC GLM do SAS, sobre a análise de variancia de dados desbalanceados, será realizado este trabalho.

Através de exemplos, sobre os quais se aplicará a teoria disponi vel para obtenção das hipóteses testadas pelos quatro tipos de somas de quadrados fornecidas pelo SAS, pretende-se traduzir esta teoria numa linguagem mais simples e acessivel.

Com isso, tem-se como principal objetivo o de informar os pesquisadores sobre a existéncia dessas hipóteses e sobre o fato de que, quando os dados são desbalanceados, suas somas de quadrados testam combinaçశes lineares dos parametros que nem sempre são aquel as esperadas.

Isto posto, pretende-se oferecer condiçбes para que o pesquisador construa e interprete as hipóteses que estão sendo testadas pelo procedimento GLM do SAS e escolha entre elas, qual a mais adequada aos propósitos de seu experimento. 


\section{REVISÃO DE LITERATURA}

YATES (1934), publica um artigo que, segundo consta na literatura, é o primeiro que realmente propre soluçб̋es razoáveis para o problema de estimar hipóteses com dados desbal anceados. Nesse artigo são apresentados três métodos:

- Método de médias năo ponderadas: um método aproximado no seritido de que as somas de quadrados para testar linhas, colunas e interaçăo, năo tem distribuiçăo exata de chi-quadrado.

- Método dos quadrados de médias ponderadas.

- Método do ajuste de constantes: através deste método Yates propz̃e uma parametrização sucessiva do modelo.

HENDERSON (1953), propZ̃e em artigo três métodos para obtenção de somas de quadrados utilizados no estudo de componentes de variáncia.

OVERALL \& SPIEGEL (1969), propõe trés métodos de análise de dados desbalanceados. Neste artigo eles generalizam e formalizam os principais métodos apresentados nos artigos anteriores. O sistema SAS incorpora em 1975 estes métodos : 
- Método do del ineamento experimental.

- Método da ordenação "a priori".

$$
\text { SPEED \& HOCKING (1976), discutem o uso da }
$$

notação RC J para dados desbalanceados. Embora haja somente uma notação $R C$, ou redução nas somas de quadrados, existem, segundo os autores, duas formas distintas pelas quais RC $\supset$ pode ser calculado.

Uma maneira \& usada por SEARLE (1971) e consiste da aplicação da notação $R C$ a modelo de posto incompleto (procedimento 1). A outra forma consiste em aplicar a notação $R C$ a modelo reparametrizado de posto completo Cprocedimento 22. Os autores discutem os pontos em que os dois procedimentos discordam.

Os autores consideram o modelo

$$
\mathbf{X}=\mathbf{X} \boldsymbol{\beta}+\boldsymbol{\theta},
$$

onde $\mathbf{Y}$ é um vetor de observaçชes de dimensão $n \times 1$;

$x$ é uma matriz conhecida de dimensão nxp de posto $q<p$;

$\beta$ é um vetor de parametros de dimensão $p \times 1$;

e é um vetor de erros de dimensão $n \times 1$.

A notação $R() \&$ definida por

$$
R(\beta)=\hat{\beta} X^{\prime} \mathbf{Y}
$$

onde $\hat{\beta}$ é al guma sol uçăo para $X^{\prime} X \hat{\beta}=X^{\prime} Y$.

Se $\mathbf{X} \in \beta$ são particionados como

$$
\left[x_{1}: x_{2}\right] \in\left[\begin{array}{l}
\beta_{1} \\
\beta_{2}
\end{array}\right] \text { e considerando-se o modelo }
$$




$$
\mathbf{Y}=\mathbf{x}_{2} \boldsymbol{\beta}_{2}+e
$$

então

$$
R\left(\beta_{2}\right)=\hat{\beta}_{2}^{\prime} X_{2}^{3} Y
$$

onde $\hat{\beta}_{2} \in$ alguma sol ução para $X_{2}^{\prime} X_{2} \hat{\beta}_{2}=X_{2}^{\prime} Y$.

Define-se:

$$
R\left(\beta_{1} \mid \beta_{2}\right)=R\left(\beta_{1}, \beta_{2}\right)-R\left(\beta_{2}\right)
$$

Neste contexto, a aplicação da notação RC $\supset$ ao modelo de posto incompleto (Procedimento 1 ) consiste em: - assumir (1) o modelo de posto incompleto; nesse caso ocorre (2). Restriçôes não estimáveis podem ser aplicadas para obter $\hat{\beta}$, mas os resultados são independentes da escolha destas restriçరes;

- assumir (3) e (4).

A aplicação da notação RC j ao modelo reparametrizado de posto completo (Procedimento 2 ocorre da seguinte forma:

- escolher um conjunto de restriçớes não estimávis e reparametrizar o modelo de posto incompleto para obter

$$
Y=X^{*} \cdot \beta^{*}+e \text {; }
$$

obtém-se então:

$$
\begin{array}{r}
\quad R\left(\beta^{*}\right)=\left(\hat{\beta^{*}}\right) \cdot\left(X^{*}\right) \cdot Y \\
\text { onde } \hat{\beta}^{*}=\left[\left(X^{*}\right) \cdot X^{*}\right]^{-1}\left(X^{*}\right)^{\prime} Y ;
\end{array}
$$

A seguir assumir $\beta^{*}=\left[\begin{array}{c}\beta_{1}^{*} \\ -\frac{1}{*}- \\ \beta 2\end{array}\right]$ e 
6.

$$
\begin{gathered}
Y=X_{2}^{*} \beta_{2}^{*}+e ; \\
R\left(\beta_{2}^{*}\right)=\left(\beta_{2}^{*}\right)^{\prime}\left(X_{2}^{*}\right) \cdot Y,
\end{gathered}
$$

onde $\hat{\beta}_{2}^{*}=\left[\left(X_{2}^{*}\right), x_{2}^{*}\right]^{-1}\left(X_{2}^{*}\right) \cdot y$.

Embora $R(\beta)=R\left(\beta^{*}\right), R\left(\beta_{2}\right)$ nao $\&$ necessariamente igual a $R\left(\beta_{2}^{*}\right)$. Neste caso o resultado pode depender do conjunto de restriçб̄es nå estimáveis usadas para completar o posto do model o.

Os autores apresentam uma comparação entre os dois procedimentos:

- No procedimento $2, R\left(\beta_{2}^{*}\right)$ nå e sempre definido e sé o $b$. pode não ser único

- Se

$$
R\left(\mu^{*}, \alpha^{*}\right), R\left(\mu^{*}, \beta^{*}\right) \in R\left(\mu^{*}, \alpha^{*}, \beta^{*}\right)
$$

såo todos definidos,

entao

$$
\begin{array}{rl}
R(\mu, \infty) & =R\left(\mu^{*}, \alpha^{*}\right), R(\mu, \beta)=R\left(\mu^{*}, \beta^{*}\right) \\
e & R(\mu, \alpha, \beta)=R\left(\mu^{*}, \alpha^{*}, \beta^{*}\right) . \\
\text { Todavia } R(\mu, \alpha, \gamma) \neq R\left(\mu^{*}, \alpha^{*}, \gamma^{*}\right) \\
\text { R }\left(\mu, \beta, \gamma^{\prime} \neq R\left(\mu^{*}, \beta^{*}, \gamma^{*}\right) .\right.
\end{array}
$$

- Em ambos os procedimentos 1 e 2 as hipóteses testadas não sæo claras. Ambós $R\left(\beta_{2} \mid \beta_{1}\right)=R\left(\beta_{2}^{*} \mid \beta_{1}^{*}\right)$ sao usados para representar as somas de quadrados a serem usadas na tabela de análise de variancia para testar algumas hipóteses sobre os paråmetros. Todavia, as hipóteses testadas podem não ser de todo óbvias. 
- As reduções $R C$ y não indicam a naturezá das hipóteses testadas.

$\mathrm{E}(\alpha \mid \mu, \beta, \gamma)=R(\beta \mid \mu, \alpha, \gamma)=0$, para o procedimento 1.

Ía se no procedimento 2 forem usadas restriçôs do tipo "soma igual à zero"

$$
R\left(\alpha^{*} \mid \mu^{*}, \beta^{*}, \gamma^{*}\right) \in R\left(\beta^{*} \mid \mu^{*}, \alpha^{*}, \gamma^{*}\right)
$$

corresporidem as somas de quadrados sugeridas por YATES (1934).

Com isto os autores concluem que tratando-se de dados desbalanceados o usuário de pacotes computacionais poderá interpretar de maneira errada as somas de quadrados por eles fornecidas se tomarem a notaçăo $R C$ I no seu valor aparente.

A hipótese geral tal como $H \beta=\gamma$ não pode ser testada usando a notação $R($ ) a qual torna a análise de dados bastante li mi tada.

Segundo SPEED, HOCKING \& HACKNEY (1978), para análise de modelos lineares de experimentos com dados balanceados existe um consenso geral sobre a análise de variância apropriada. A análise de dados desbalanceados é geral mente baseada numa extensão dos métodos bal anceados. As várias formas de realizar análises desbalanceadas podem levar a diferentes resultados. O propósito destes autores é rever os métodos de análise mais comumente citados na literatura e relacionar as somas de quadrados associadas as hipóteses lineares a serem testadas. 
Um critério natural para a seleção do método de análise parece ser a apropriação da hipótese a ser testada. Outros fatores como a facilidade de cálculos e a ortogonalidade das formas quadráticas não podem ser boas justificativas se a hipótese testada não tem significado prático.

Os autores incorporam dois métodos de HENDERSON (1953) ao rol de métodos utilizados em análise de variáncia para dados desbal anceados:

- Método 1 de Henderson deriva do método do ajuste de constante de Yates.

- Método 3 de Henderson é conhecido como método da or denação "a priori".

Apresentam também os métodos usados pelo SAS:

- Método I SAS 76: é similar ao método do ajuste de constantes no qual $\mathrm{H}_{2} \in \mathrm{H}_{2}$ Capresentadas na Tabela 1 ) são as hipoteses para efeitos principais. Consiste do método de ordenação a priori de OVERALL \& SPIEGEL (1969).

- Método II SAS 76: é o mais comum dos procedimentos, baseado no método do ajuste de constantes no qual as somas de quadrados ajustadas, $R C \alpha \mid \mu, \beta)$ e $R(\beta \mid \mu, \alpha)$ são usadas para testar efeitos principais. Este é o método do delineamento experimental de OVERALL \& SPIEGEL (1969) no qual as hipoteses $\mathrm{H}_{3} \in \mathrm{H}_{2}$ (apresentadas na Tabela 1 ) são testadas.

- Métodos III e IV SAS 76: estes dois métodos são equi valentes ao método dos quadrados ponderados de médias ou 
8.

minimos quadrados completos de OVERALL \& SPIEGEL (1969) no qual as hipóteses dos efeitos principais são $\mathrm{H}_{1} \in \mathrm{H}_{5}$ Capresentadas na Tabela 1 ).

SPEED, HOCKING \& HACKNEY (1878), também ci tam as hipóteses mais comuns sobre linhas, colunas e interação em termos do modelo

$$
y_{i j k}=\mu_{i j}+e_{i j k}
$$

Estas são apresentadas Tabela 1.

Tabela 1 - Hipóteses Associadas ao Modelo de Classificação Dupla.

Efeitos

Linhas

$$
\begin{aligned}
& H_{1}: \bar{\mu}_{i}=\bar{\mu}_{i} \cdot . \\
& H_{2}: \sum_{j} n_{i j} \mu_{i j}, n_{i}=\sum_{j} n_{i}, j \mu_{i}, j, n_{i} \cdot \\
& H_{3}: \sum_{j} n_{i j} \mu_{i j}=\sum_{i} \sum_{j}\left(n_{i j} n_{i j} \mu_{i, j} j, n_{j}\right. \\
& H_{4}: \mu_{i 1}=\mu_{i}, 1
\end{aligned}
$$

Col unas

$$
\begin{aligned}
& H_{5}: \bar{\mu}=\bar{\mu}_{. j}, \\
& H_{0}: \sum_{i j} n_{i j} \mu_{i j}, n_{j j}=\sum_{i} n_{i j}, \mu_{i j},<n_{j j} \\
& H_{7}: \sum_{i j} n_{i j} \mu_{i j}=\sum_{j,} \sum_{i}\left(n_{i j} n_{i j}, \mu_{i j}, J, n_{i} .\right. \\
& H_{0}: \mu_{1 j}=\mu_{1 j} .
\end{aligned}
$$

Interação $H_{\phi}: \mu_{i j}-\mu_{i}{ }_{j}-\mu_{i j},+\mu_{i j},=0$

หุ. 
As hipoteses $\mathrm{H}_{1}, \mathrm{H}_{2}, \mathrm{H}_{3} \in \mathrm{H}_{4}$ referem-se ao efeito principal de $A$, suas somas de quadrados são usadas para mensurar o efeito do fator $A$. As hipoteses $H_{1}, H_{2} e H_{9}$ coincidem se $n_{i j}$ igual a $n$ para todo $i j$. A hipótese $H_{1} \hat{k}$ fácil de interpretar desde que não haja diferença entre os niveis do fator A quando considerados os niveis de $B$. As hipóteses $\mathrm{H}_{2} e \mathrm{H}_{9}$ não são fáceis de interpretar e representam comparaçర̃es entre médias ponderadas com os pesos sendo funçб̃es das frequéncias de caselas. A hipótese $H_{4}$ distinta das tres primeiras e considera somente as comparaçơes entre médias de caselas da primeira coluna. As hipóteses $H_{5}, H_{6}, H_{7}$ e $H_{8}$ são correspondentes à $H_{1}, H_{2}, H_{9} e$ $H_{4}$, respectivamente, associadas ao fator $B . H_{0} E$ a hipótese da interação e é comum a todos os métodos, com $\mathbf{n}_{i j}$ maior que zero. A suposição de $n_{i j}$ maior que zero não é essencial para todos os métodos. Por exemplo, o método de ajuste de constantes requer apenas que o delineamento seja conexo. As hipóteses são válidas considerando-se $n_{i j}$ igual a zero, quando não há observação na casela $(i, j)$. Todavia, a hipótese associada com o método dos quadrados de médias ponderadas só é válida se $n_{i j}$ maior que zero, para todo $i, j$. Segundo os autores alguns programas empregam uma modificação no procedimento $R C)$, fixando $\boldsymbol{\gamma}_{i j}$ igual a zero se $n_{i j}$ igual a zero. Isto facilita o calculos das somas de quadrados mas não fornece indicação de quais hipóteses estão sendo testadas. As somas de quadrados obtidas através 
do SAS 76 tipos III e IV não necessariamente coincidem quando há caselas vazias. O tipo III também emprega a modificação acima e o tipo IV não o faz.

URQUHART \& WEEKS (1978), afirmam que com dados incompletos raramente se conhece o modelo linear apropriado. O modelo de médias é uma alternativa robusta a assumir-se um modelo superparametrizado inapropriado. Os autores apresentam um experimento com dois fatores: raças (representadas por $\beta_{i}{ }^{2} e$ dietas (representadas por $\boldsymbol{\gamma}_{j}$ de acordo com o modelo

$$
\begin{gathered}
y_{i j k}=\mu+\beta_{i}+\gamma_{j}+e_{i j k} \\
i=1,2,3 \\
j=1,2,3,4 \\
k=0,1, \ldots, n_{i, j}
\end{gathered}
$$

e consideram seis padröes de frequencias de caselas para este mesmo experimento, que consistem de um conjunto aditivo de médias de caselas e cinco conjuntos não aditivos.

Através da obtenção da estimativa da diferença entre raças um e tres, isto $e,\left(\widehat{\beta_{1}-\beta_{9}}\right)$, para todos estes casos eles observam que:

- 0 padrão de frequéncias de caselas (padrão n) não influencia a $E\left(\widehat{\beta_{1}-\beta_{9}}\right)$ se ocorre aditividade;

- Da mesma forma, $E\left(\widehat{\beta_{1}-\beta_{9}}\right.$ não muda entre os conjuntos de médias se o padrão $n$ é bal anceado.

- Por outro lado, se o modelo é inadequado, o padrão $n$ e as médias de caselas, ambas influenciam a $E\left(\widehat{\beta_{1}-\beta_{9}}\right.$. 
A questão é desejar-se um estimador de variáncia minima que não estime algo de interesse se um modelo incorreto for assumido, ou um estimador que estime algo de interesse sobre um modelo no qual suposiçชes são facilmente defendidas, mas talvez com uma variancia maior. Confiar em assumir-se um modelo linear de posto incompleto pode levar a problemas. Os parámetros $\mu_{i}$ do modelo de médias tem uma relação clara com o contexto experimental e as médias amostrais correspondentes proporcionam estimativas para eles. Além disso, alguma função linear dos $\mu_{i}$ pode ser também estimada. Neste caso para obter-se a diferença entre as médias, utilizar-se-a a diferença entre as médias da linha um e as médias da linha trés.

Em resumo, os autores encorajam os pesquisadores e estatisticos a abordarem a análise de dados desbalanceados definindo e estudando combinaçőes lineares das médias de caselas de interesse.

BURDICK \& HERR (1980), afirmam que até então, a questão da análise de dados de classificação dupla desbal anceada năo foi respondida de forma satisfatoria. Pelo contrário, esta questão ainda provoca controvérsias entre os estatisticos. Seu propósito não é o de solucionar estas controvérsias, mas sim, discutir as respostas sugeridas.

Desenvol vem, através da notação geométrica, sete hipóteses associadas a linhas, colunas e interação. HR1 (HC1) é a hipótese de que médias de linhas (colunas) são 
iguais; HRZ (HCZ) é a hipótese de não existéncia de efeito de linhas (colunas) não ajustadas; HR3 (HC3) é a hipótese de não existéncia de efeitos de linhas (colunas) ajustadas para colunas (linhas); HRC é a hipótese de não existéncia do efeito de interação entre linhas e colunas.

Destacam duas abordagens para a análise de dados desbalanceados de classificação dupla: a comparação de modelos e a abordagem parametrica, que se concentra na interpretação paramétrica das hipóteses de efeitos principais. A desvantagem da abordagem paramétrica é a não ortogonalidade das hipóteses, gerando somas de quadrados que somadas não resultam na soma de quadrados dos parametros (HR1, HC1 e HRC). A mais séria consequéncia da não ortogonalidade e a possibilidade de que a deteç̧ão de efeitos significativos pode nåo ser observada.

A comparação de modelos consiste na comparação do "modelo completo" com o "modelo reduzido" o qual resultaria se a hipótese fosse verdadeira, isto é, sob $\mathrm{H}_{0}$. Seguidores da comparação de modelos neste caso, defendem HR2, HR3, HC2 e HC3 como hipóteses de efeitos principais. A desvantagem deste método é que a interprétaçăo paramétrica de HR2, HR3, HC2 e HC3 depende do tamanho das caselas.

Um modelo quase aditivo obtido justificando-se a pressuposiçăo de năo interaçăo. Para este modelo eles enunciam o seguinte teorema:

"Num modelo quase aditivo de classificaçăo dupla a hipótese 
sobre linhas ajustadas para colunas é verdadeira se e somente se as médias de linhas são iguais. Se esta hipótese e falsa, então na auséncia de interação o teste estatistico de linhas ajustadas para colunas tem o mesmo poder de um simples teste estatistico para médias de linhas."

FREUND (1980), menciona que a grande disponibilidade de computadores tem permitido a aplicação da metodologia de modelos lineares na analise de dados desbalanceados e que os programas estatisticos, que usam estas metodologias , são apropriados para resolver os problemas onde há caselas vazias, porém nunca se esquecendo da 1 i mitação existente neste tipo de sol ução.

A ocorréncia de caselas vazias tem sido um problema muito discutido. Metodologias especificas tem sido desenvolvidas para certos casos, no entanto, nenhuma é uni versalmente aceita.

Segundo FREUND (1980), o procedimento GLM do SAS usa a metodologia de funçơes estimáveis, provendo um mecanismo para verificação das bases das estimativas obtidas. 0 procedimento tambem prove metodos pelos quais diferentes resultados podem ser obtidos ilustrando o fato de que não há somente uma resposta correta para o caso de caselas vazias. As funçశes estimáveis são uma classe especial de funçơes dos parametros. 0 procedimento obtém uma sol ução através da inversa generalizada : $\hat{\gamma}=\left(X^{\prime} X\right)^{-} X^{\prime} y$.

As funçơes estimáveis são obtidas construindo-se um vetor 
de constantes 1 tal que $l^{\prime} \gamma$ é unicamente estimável.

A análise de dados com frequéncias desiguais de caselas é afetada pelo fato de que estimativas de alguns parámetros podem conter outros parámetros sem interesse. 0 PROC GLM reconhece este dilema e proporciona informaçơes sobre os quatro tipos de funçỡes estimáveis correspondendo à quatro "filosofias" para produzir somas de quadrados. As funçర̃es estimáveis tipos III e IV produzem diferentes resultados quando há caselas vazias. 0 conjunto de estimativas à escolher depende do seu sentido com relação a estrutura dos dados. As funçơes estimáveis tipo IV podem produzir diferentes estimativas e somas de quadrados se os dados forem reorganizados.

A localização das caselas vazias não afeta a estimativa e soma de quadrados tipo III.

Os autores concluem que os resultados obtidos nas análises de dados com caselas vazias são bastante confusos, o que dificulta a obtenção de funçỡes usuais aos pesquisadores. Isto é coerente pois a presença de caselas vazias deixa uma lacuna nos dados, impossibilitando a estimativa dos parámetros desejados. Não há como sustentar que o PROC GLM tenha a solução correta para estas situaçర̃es, todavia, ele prove uma informação sobre a natureza das estimativas possiveis e cabe ao pesquisador decidir entre os resultados qual tem aplicação prática.

GOODNIGHT (1980), diz que usando o conceito 
de estimabilidade, testes de hipóteses em modelos lineares fixos com muitos fatores são desenvolvidos sem recorrer as "pressuposiçס̃es usuais". Ele define três tipos de funçס̃es estimáveis, um tipo é caracterizado pelo número desigual de observaçơes por casela, outro pela presença de caselas vazias e um terceiro caso quando ocorre algum grau de confundimento para algum modelo linear de efeitos fixos Segundo o mesmo autor. no campo dos modelos lineares aparece a notação $R C$, que calcula a redução em soma de quadrados devido a um efeito especifico fixando todos os efeitos exceto aqueles que contém o efeito especificado sem reparametrizar o modelo. Por outro lado a notaçăo $R^{*} C \cdot$ J reparametriza o modelo usando a pressuposiçăo usual e calcula a redução de somas de quadrados devido a um efeito especifico fixando todos os outros efeitos.

- autor apresenta o conceito de estimabilidade dado pelo manual do SAS e diz que alguns possiveis conjuntos geradores para uma funçăo linear esti mável dos parâmetros (L) são:

$-\mathbf{x}$

$-X^{\prime} X$

- As matrizes $X ' X$ Forward Doolitle ou Cholesky;

$-(x \cdot x)^{-} x \cdot x$

- A forma canónica de Hermite de $X^{\prime} X$;

- Alguma matriz produzida por operaçб̃es nas linhas em al gumas das mencionadas acima. 
Um número infinito de funçס̃es estimáveis $L$ pode ser construido dos conjuntos geradores acima. Para um determinado modelo um conjunto de funçỡes estimáveis L pode ser gerado, um L para cada efeito no modelo.

De acordo com o autor tres tipos de funçơes estimáveis podem ser descritas. Tipos I e II são para o campo $R C$ J e tipo III é para o campo $R^{*} C$ J. A forma por ele apresentada para obtenção das funçơes estimáveis tipo I de um determinado modelo, consiste em: calcular-se a matriz de Forward Doolitle para $X ' X$, e tomar-se o L para cada efeito, como as linhas não nulas associadas com este efeito.

As funçơes estimáveis $L$ tipo I produzem formas quadraticas ortogonais que, devido a estrutura dos erros, săo independentes. Todavia, as hipóteses tipo I nå são invariantes com respeito a ordem dos efeitos no modelo e para delineamentos desbalanceados, cada hipótese envolve parámetros do efeito testado acrescido de todos os outros parámetros que o seguem na especificação do modelo. Além disso, as hipóteses tipo I são dependentes das frequencias de caselas.

Segundo GOODNIGHT (1980), a construção das funçơes estimáveis tipo II se baseia na seguinte definição de efeito contido:

"Um efeito $E_{2}$ é dito conter um efeito $E_{1}$ se todas as colunas da matriz $X$ associadas com $E_{1}$ podem ser representadas como combinaçơes 1 ineares das colunas associadas com $E_{2}$." 
18.

No modelo sem restrição nos parámetros, se $E_{2}$ contém $E_{1}$ então $R\left(E_{1} \mid E_{2}\right)=0$. E claro então, que as funçర̋es estimáveis $L$ tipo II para cada efeito envolvem somente os parametros deste efeito e do efeito que o contém. A correta ordenação do modelo deve ser feita e a matriz de Forward Doolitle para $X^{\prime} X$ calculada. As funçros estimáveis $L$ tipo II para um efeito $E_{1}$ são as linhas da Doolitle associadas com o modelo que foi rearranjado colocando-se todos os efeitos que não contém $E_{1}$ antes de $E_{1}$.

GOODNIGHT (1880) acrescenta que, a hipótese tipo III para delineamentos sem caselas vazias corresponde as funçర̃es estimáveis usadas no caso balanceado. Ao contrário das tipo I e II, as funçб̋es estimáveis tipo III não dependem da frequéncias de caselas. Elas são definidas da seguinte forma: um conjunto de funçశ̋es estimáveis $L$, um para cada efeito no modelo, são do tipo III se cada L é uma hipótese de posto máximo envol vendo somente os parámetros do efeito em questão e parámetros de efeitos que contém estes e cada $L$ é ortogonal a todas as funçơes estimáveis $L$ de efeitos que contém o efeito em questão.

HOCKING, SPEED \& COLEMAN (1980), dizem que a escolha da analise para um modelo de efeitos fixos com dados desbalanceados é ilimitada. O usuário da maioria dos pacotes estatisticos pode obter várias tabelas de análise de variáncia especificando diferentes opçశ̋es e/ou mudando a ordem dos termos no modelo. Isto leva os usuários a ficarem 
confusos sobre qual conjunto de somas de quadrados é apropriada. Neste artigo os autores se proprem a prover um critério para escolha da hipótese a ser testada no caso desbalanceado e apresentam um algoritmo para obtenção da hipótese desejada. Oferecem a base teórica para testar-se as referidas hipóteses: se o modelo é caracterizado em termos do vetor de parametros $\mu$ e uma hipotese na forma H igual a zero é proposta, entao o teste estatistico é dado por

$$
F_{(q, n-p)}=\frac{S Q(H)}{q s^{2}}
$$

onde $q \in e$ posto de $H, s^{2} \in$ o quadrado médio residual com n-p graus de liberdade e a soma de quadrados do numerador $S Q(H)$ é dada por

$$
S Q(H)=(H \boldsymbol{p}) \cdot\left[H \text { V }(\boldsymbol{p}) H^{\prime} / \boldsymbol{\sigma}^{2}\right]^{-1} H \boldsymbol{p}
$$

HOCKING, SPEED \& COLEAMN (1980), colocam que, em parte dos programas computacionais a matriz de hipóteses $H$ não é especificada e a SQCHJ não é calculada diretamente. Não haveria objeção à aproximação se a hipótese efetiva fosse conhecida e razoável. O SAS gera a matriz de hipóteses usando um algoritmo computacional. Isto e ao menos um passo para a decisão correta.

Segundo os mesmos autores, alguns procedimentos computacionais produzem hipóteses que dependem da frequéncia de caselas e são difíceis de interpretar. Outros procedem como se estivessem construindo um modelo 
considerando submodelos de crescente complexidade através do qual testam as hipóteses. Aqui, os autores alertam para o fato de que estas somas de quadrados são frequentemente usadas para testes com efeitos principais em situaçōes nas quais o modelo inclui a inter ação.

No caso de todas as caselas não nulas, estes autores recomendam que se use as mesmas hipóteses que seriam testadas se todas as frequencias de caselas fossem as mesmas. Quando ocorrem caselas vazias eles propóe um procedimento para determinar hipóteses razoáveis a serem testadas. O procedimento é baseado na premissa de que o pesquisador tem em mente as hipóteses que são apropriadas se todas as casel as são completas.

HOCKING, SPEED \& COLEMAN (1980), partem do modelo

$$
\begin{aligned}
& Y=\mathbf{W}+\mathbf{e} \\
& \text { sujeito a } G \boldsymbol{\mu}=0
\end{aligned}
$$

onde $\boldsymbol{\mu} \in$ o vetor de dimensão $\mathrm{P}$ de médias de caselas das popul ações incluidas no estudo;

W é a matriz de contagem tal que W'W é diagonal com - i-ésimo elemento da diagonal igual ao número de observaçơes na i-ésima popul ação;

$G \in$ uma matriz rXp de posto $r$, tal que $G \mu=0$ represente um conjunto de relaçóes lineares entre médias de casel as.

Particionam o vetor $\boldsymbol{\mu}$ em $\boldsymbol{\mu}_{m}$ e $\boldsymbol{\mu}_{0}$ correspondendo $s$ 
respectivamente as medias de caselas para caselas vazias e observadas. Obtém-se as hipóteses efetivas construindo-se as hipóteses para o caso desbalanceado através das hipóteses que seriam testadas se o experimento fosse balanceado, colocando-se as médias de caselas que não podem ser estimadas, em função das que podem ser estimadas. Os autores consideram que a aproximação feita neste metodo é consistente e baseada em principios fundamentais, tem mérito considerável sobre aproximaçơes baseadas somente em critérios computacionais.

GOODNIGHT (1980), diz que usando o conceito de estimabilidade, testes de hipóteses em modelos lineares fixos com muitos fatores são desenvolvidos sem recorrer às "pressuposiçర్s usuais". Ele define tres tipos de funçర̃es estimáveis, um tipo é caracterizado pelo número desigual de observaçбes por casela, outro pela presença de caselas vazias e um terceiro tipo quando ocorre algum grau de confundimento para algum modelo linear de efeitos fixos.

SEARLE (1980), ressal va que o PROC GLM do SAS inclui em sua salda quatro tipos de funçชes estimáveis que possuem um componente arbitrário (representado pela letra L) nos seus coeficientes. Ele mostra como tais funçช̃es estimáveis arbitrárias săo derivadas da conhecida expressão geral para hipoteses testadas pela tradicional estatistica F, na análise de variancia efetuada para dados balanceados. Segundo ele os programas de computadores 
22.

oferecem cálculos da estatistica F facilmente obtidos, sem formulação da hipótese a ser testada anteriormente. As hipóteses testadas por estas estatisticas $F$ podem não ter uso ou interesse.

SEARLE (1980) acrescenta ainda que as somas de quadrados entituladas tipos I, II e III fornecidas pelo PROC GLM do SAS são interpretadas em termos das funçర̃es estimáveis que são bases para as hipóteses testadas pelas somas de quadrados. Entretanto, estas funçర̃es estimáveis são mais informativas que os valores $R C$ J. Como estas somas de quadrados são facilmente obtidas pelos usuários do programa, se faz necessária uma descrição do seu significado. Esta descrição pode ser feita em termos das expressర̃es explicitas para as hipóteses testadas, usando funçỡes lineares dos parámetros do modelo com coeficientes que são funçชes dos números de observaçర̃es por casela ou em termos das funçб̄es estimáveis tendo coeficientes que envolvam valores arbitrários.

Segundo o mesmo autor, as funçช̃es estimáveis tipos I, II, III e IV fornecidas pelo SAS consistem de funçơes de valores $\mathrm{L}$ ao lado de cada parámetro do modelo. Multiplicando-se estes valores $L$ pelos seus parametros correspondentes e somando-se os produtos, obtém-se uma função estimável $f$. Correspondendo a $f$ há uma soma de quadrados SS(f). Segundo SEARLE (1980), diz-se que "f explica SS(f)". 
- autor apresenta as seguintes somas de quadrados SS(f), explicadas desta forma:

- Tipo I: valores $R C$ J sequenciais;

- Tipo II: valores RC I para cada fator ajustado para todos os outros que não o contém;

- Tipo III: valores RC J para cada fator ajustado para todos os outros.

Ao contrário dos tipos I, II e III o procedimento tipo IV não pretende proporcionar funçర̋es estimáveis que expliquem al guma soma de quadrados pré definida. Ele fixa uma hipótese e calcula a estatistica F para testa-la.

SEARLE (1980), descreve para modelos com e sem interação, com caselas vazias, um procedimento para se obter os contrastes para cada efeito e os quatro tipos de funçס̃es estimáveis. Atraves deste critério se irá seguir a sequéncia lógica de primeiro se construir uma hipótese de interesse e então testá-1a. A hipótese de interesse pode ser testada em termos das funçơes esti máveis do SAS.

FREUND (1980), ressal va que o procedimento GLM do SAS (SAS Institute, 1979 ) usa a metodologia de funçชes estimáveis para se verificarem as bases para as estimativas obtidas.

MILLIKEN \& JOHNSON (1984), afirmam que quando todas as combinaçơes de tratamentos são observadas, as hipóteses tipo III são as mesmas testadas quando os dados são balanceados. Quando alguma combinação de tratamentos é 
perdida, todavia, tais hipóteses não podem ser testadas pois el as envolvem parametros sobre os quais não se tem informação. Ambas as hipóteses tipos I e II para efeitos principais dependem do número de observaçôes por casela. Se hâ ao menos uma observação em uma determinada casela, então essa média de casela é estimável. o desenvolvimento de hipóteses tipo III não depende do tamanho das caselas mas somente de quais casel as são observadas. Eles não discutem a construção de hipóteses tipo III para o caso com caselas vazias, mas afirmam ser a tipo III a pior hipótese a considerar-se nesta situação porque eles não vêem uma forma razoável de interpreta-la.

Segundo esses autores, as hipóteses tipo IV são construidas de tal forma que os coeficientes de médias de caselas sejam balanceados e por isso as hipóteses resultantes são interpretáveis. Basicamente, para uma estrutura de tratamentos de classificação dupla, a hipótese tipo IV compara os niveis de um tratamento sobre um ou mais niveis comuns do outro. 0 PROC GLM do SAS gera algumas hipoteses tipo IV que podem ser interpretadas, mas uma interpretação apropriada não pode ser feita sem primeiro examinar-se as funçớes estimáveis tipo IV, para ver que hipóteses são geradas e testadas. Isto é, não há uma única interpretação aproapriada para todos os conjuntos de dados com combinaçơes ou tratamentos perdidos. Reordenando os tratamentos pode-se obter diferentes hipoteses tipo IV e 
25.

portanto, diferentes somas de quadrados e testes F. Então, a análise tipo IV obtida depende de como os tratamentos são chamados ou como eles são numerados.

SEARLE (1987), menciona que vários pacotes computacionais podem, atualmente, realizar cálculos de modelos lineares para grandes conjuntos de dados, incluindo aqueles com número desigual de observaçס̃es nas subclasses (dados desbalanceados). Interpretar as saidas dos pacotes para estes dados de maneira análoga às saldas provenientes de dados de experimentos bem executados cujos delineamentos são bem conhecidos, pode levar a erros. Ele define dados desbalanceados como os dados nos quais o número de observaçб̃es nas caselas não é igual, podendo incluir casel as sem observação. Ao contrário do desbalanceamento planejado, aqui as caselas vazias ocorrem de maneira não planejada.

- autor afirma que a classe de dados desbalanceados tem duas subdivisð̄es. Uma, quando todas as caselas contém dados; outra, quando existe alguma casela vazia. Denomina-as dados com todas as caselas completas e dados com algumas caselas vazias, respectivamente. Com relação aos métodos de análise utilizados pelos pacotes computacionais o autor afirma que, primeiramente, para dados desbalanceados não é oferecido um método único de análise, que não apresente ambiguidade. Além disso, di versos métodos estão disponiveis, métodos estes que não são facilmente interpretados como os métodos para dados balanceados; não 
26.

são bem conhecidos nem amplamente divulgados. Em segundo lugar, como consequéncia de se terem diversos métodos de análise, nem todos os pacotes estatisticos fazem necessariamente as mesmas análises sobre um mesmo conjunto de dados desbalanceados. Consequentemente, no contexto de hipóteses testáveis e obtenção de somas de quadrados em uma análise de variáncia padrăo, eles fornecem, para um mesmo conjunto de dados, uma variedade de somas de quadrados. 0 problema é identificar qual delas é proveitosa.

Um importante resultado para o modelo de classificação dupla sem interação, é que quando os dados são conexos, todas as médias de caselas são estimáveis. Ao contrário, no modelo com interaçăo, somente săo estimáveis as medias de caselas que não são vazias, seu melhor estimador linear năo viesado CBLUE: Best Linear Unbiased Estimators é dado por

$$
\boldsymbol{P}_{i j}=\bar{y}_{i j} \text {, para } n_{i j} \neq 0 \text {. }
$$

Com relação aos quatro tipos de somas de quadrados, SEARLE (1987), afirma que o objetivo do SAS em prover os diferentes tipos de somas de quadrados é originado da filosofia fundamental da análise da variancia. Os quatro conjuntos de somas de quadrados chamadas tipos I, II, III e IV e caracterizadas, respectivamente como sequencial, cada um após todos as outros, $\Sigma$ restriçőes e hipoteses. Igualdade entre os tipos existem sob as seguintes circunstancias: $I=I I=I I I=I V$ para dados bal anceados; 


$$
\begin{aligned}
\text { II }= & \text { III = } \\
\text { IV }= & \text { IV } \text { Para modelos sem interação; } \\
& \text { O autor apresenta uma breve discussão sobre o }
\end{aligned}
$$

quatro tipos:

- Tipo I: a sequéncia com que os fatores do modelo são especificados a rotina, na entrada dos dados determina a sequéncia das somas de quadrados tipo I. A sequéncia de salda das somas de quadrados corresponde precisamente a sequéncia de entrada dos fatores, devendo fatores de efeitos principais preceder interaçőes e também, fatores que têm outros hierárquicos com eles preceder os fatores hierárquicos. Então quando fatores são linhas, colunas e interação $e$ são identificados na ordem $A, B, e A * B$, respectivamente, as somas de quadrados tipo I são

$$
R(\alpha \mid \mu), R(\beta \mid \mu, \infty) R C \alpha * \beta \mid \mu, \alpha, \beta)
$$

- Tipo II: as somas de quadrados chamadas tipo II são similares a algumas do tipo I. Elas são do tipo descrito como RCcada fator/todos os outrosj. Para a classificação dupla as somas de quadrados tipo II são $R C \alpha \mid \mu, \beta), R C \beta \mid \mu, \infty$ e $R(\cot \beta \mid \mu, \alpha, \beta)$. Na verdade $R C$ um fator/todos os outros apropriadosJ é mais adequado, onde "apropriado" significa que esta soma de quadrados e para algum fator ajustado para todos os outros fatores exceto interaçбes que envol vem este fator e fatores hierárquicos com este fator. Por exemplo, uma soma de quadrados tipo II devido a $A$ é uma soma de quadrados ajustada para todos os outros fatores e interação, 
28.

exceto para interaçőes envolvendo $A$ e para fatores hierárquicos com $A$.

- Tipo III: estas são as somas de quadrados para modelos com restrição $\Sigma$ Equivale a:

$$
R(\dot{\alpha} \mid \dot{\mu}, \dot{\beta}, \dot{\gamma}) \quad \text { e } R(\dot{\alpha} \mid \dot{\mu}, \dot{\beta}, \dot{\gamma}) \neq R(\alpha \mid \mu, \beta, \gamma)=0
$$

Além disso, para dados com todas as caselas completas, as somas de quadrados tipo III são idénticas as da análise de quadrados de médias ponderadas.

- Tipo IV: somas de quadrados tipos I, II e III são derivadas do ajuste de um modelo e diferentes submodelos deste. Mas as somas de quadrados tipo IV são para testar hipóteses que são determinadas pela própria rotina GLM do SAS. A hipótese selecionada depende do padrão de caselas completas, e então a estatistica $F$ está de acordo com a prática estatistica de arranjar uma hipótese e testá-la. Um exemplo é que a soma de quadrados tipo IV para linhas é a soma de quadrados do numerador para uma hipótese que consiste de contrastes entre médias de caselas para caselas completas que estão na mesma coluna e começando com aquelas que estão na última linha. Tais hipóteses dependem de quais caselas possuem dados e quais niveis do fator colunas são definidos na última linha. Em geral, hipóteses determinadas desta maneira não são necessariamente de interesse. Além disso, as somas de quadrados tipo IV para linhas, por exemplo. podem diferir de uma sequéncia de linhas para outra, para o mesmo conjunto de dados. Então, o que é 
29.

chamado de soma de quadrados tipo IV para linhas não é único, para um determinado conjunto de dados com algumas caselas vazias e as hipóteses correspondentes também não são únicas.

As saldas do GLM do SAS incluem uma forma especial de funçỡes estimáveis para cada um dos tipos I, II. III e IV. A utilidade destas funçZ̃es estimáveis é que cada uma proporciona uma base para o usuário ser capaz de expor as hipóteses associadas a cada soma de quadrados.

o mesmo autor apresenta algumas consideraçơes sobre estimabilidade. O BLUE de uma função estimável q' $\beta$ é $\hat{q^{\prime} \beta}=q^{\prime} \beta^{\circ}$

sendo $\beta^{\circ}$ qualquer vetor solução das equaçžes normais.

SEARLE (1987), afirma que o SAS GLM usa uma inversa generalizada $G$, de $X ' X$ correspondente ao vetor sol ução. G é sempre simétrica e reflexiva e é chamada Ga na salda, para indicar que esta satisfaz as duas primeiras condiçб̃es de Moore-Penrose. Além disso, a $G$ sempre corresponde à restriçơes de que alguns elementos na solução são zeros. $X^{\prime} X$ e $G$ podem ser obtidas nas saldas. Obter $G$ é essencial para o conhecimento de qual vetor solução é estimado e torna hábil deduzir os erros padrão das esti mati vas.

Ainda segundo SEARLE(1987) os valores

numericos do vetor solução são dados por $\beta^{0}=G^{\prime} y$ das equações normais $X^{\prime} X \beta^{\circ}=X^{\prime} Y$ 
30.

e ele pode não ser 0 BLUE de $\beta$ cque em modelos superparametrizados, pode não existir).

$$
E\left(\beta^{0}\right)=H \beta \text { para } H=\left(X^{\prime} X\right)^{02}\left(X^{\prime} X\right)
$$

Portanto, BLUE de $H \beta=\beta^{\circ}$

Através de $\mathrm{H} \beta$ se obtém as $\mathrm{f} u n c ̧ \widetilde{e s}$ paramétricas para as quais $\beta^{\circ} \in$ BLUE.

A matriz de variancias e covariancias de $\beta^{\circ}$ é dada por

$$
\operatorname{var}\left(\beta^{\circ}\right)=G X^{\prime} X G^{\prime} \sigma^{2}=G \sigma^{2}
$$

esta última igualdade porque uma $G$ simetrica e reflexiva usada.

Entåo $\hat{\operatorname{var}}\left(\beta^{\circ}\right)=G \sigma^{2}$.

- Manual do SAS, Capitulo 9 (1990), apresenta - conceito de "Estimabilidade", com o qual o pacote irá trabalhar na construção dos quatro tipos de funçzes estimáveis que ele of erece:

Para modelos lineares tais como

$$
Y=X B+C
$$

que têm $E(Y)=X B$, o objetivo inicial é estimar ou testar os elementos de $B$ ou alguma combinação linear desses elementos. Isto é feito calculando combinaçres lineares dos Y's observados. Para estimar uma função linear dos B's, digamos LB, deve-se ser capaz de encontrar uma combinaçăo linear dos Y's que tem como valor esperado LB. Temos, 
portanto, a seguinte definiçæo, dada por RAO', citado pelo Manual do SAS, Capitulo 9 (1990):

LB estimável se e somente se existe uma combinação linear dos elementos do vetor de observaçôes $Y$ cujo valor esperado é LB.

Qualquer combinação linear dos elementos do vetor de observaçós $Y$, por exemplo K Y, terá como valor esperado, $E(K Y)=K X$ B. Assim, o valor esperado de qual quer combinação linear dos elementos do vetor de observaç̋̃es Y á igual aquela mesma combinação linear das linhas de $\mathrm{X}$ multiplicadas por B. Portanto:

LB estimável se e somente se uma combinação linear das Iinhas de X que é igual a L pode ser encontrada.

Assim, segundo o Manual do SAS, Capitulo $\theta$ (1890), as linhas de $\mathrm{X}$ formam um conjunto gerador do qual um $L$ pode ser construído. Como $x$ pode ser construída das linhas de $X^{\prime} X$, isto é, $X=\left[X\left(X^{\prime} X\right)^{-}\left(X^{\prime} X\right)^{\prime}\right]$, as linhas de $X^{\prime} X$ formam também um conjunto gerador do qual todas as funçס̃es estimaveis $L$ podem ser construidos. Similarmente, as linhas de $\left(X^{\prime} X\right)^{-} X^{\prime} X$ formam tamberm um conjunto gerador para $L$.

Portanto, se L e gerado como uma combinação linear das linhas de $X, X^{\prime} X$ ou $\left(X^{\prime} X\right)^{-} X^{\prime} X,{ }^{\prime}$ LB é estimável.

${ }^{1}$ RAO, C.R. On the linear conbination of observations and the
general theory of least equares. sankhyã,
1945. 
Uma vez que um $L$, de posto linha completo tenha sido formado de um conjunto gerador, LB pode ser estimado por $L b$, onde $b=\left(X^{\prime} X\right)^{-} X \cdot Y$. Da teoria geral de modelos lineares, Lb é o melhor estimador imparcial de LB.

De acordo com o Manual do SAS, Capítulo 9 (1990), surge a questão: com muitos conjuntos geradores disponíveis, qual o melhor para representar L? Naturalmente, um conjunto gerador contendo o minimo de linhas cde posto linha completos e um máximo de elementos nulos é desejável. Por isso, o PROC GLM calcula uma inversa generalizada (G2) de $X^{\prime} X$ tal que $\left(X^{\prime} X\right)^{02} x^{\prime} X$ contém usual mente numerosos zeros e tal que as linhas não nulas são linearmente independentes. - GLM usa as linhas não nulas de $(X \cdot X)^{62} X \cdot X$ para representar L.

IEMMA (1993), apresenta as hipóteses mais comuns sobre linhas, colunas e interação. Em geral, para tratar efeitos de linhas (Fator $A$ exitem quatro hipóteses mais comuns:

- hipóteses sobre médias ponderadas de linhas;

- hipóteses sobre médias ponderadas de linhas ajustadas para col unas;

- hipóteses sobre médias não ponderadas de li nhas;

- hipóteses sobre médias não ponderadas de linhas, segundo as caselas vazias.

De modo análogo, para testar os efeitos de col unas: 
- hipóteses sobre médias ponderadas de colunas;

- hipóteses sobre médias ponderadas de colunas ajustadas para linhas;

- hipóteses sobre médias não ponderadas de col unas;

- hipoteses sobre médi as não ponderadas de col unas, segundo as caselas vazias.

A ocorréncia de caselas vazias pode conduzir a graves problemas em relação à estimação de funções parametricas e, portanto, à testabilidade dessas funções. Estes problemas se refletem sobre a interpretação das verdadeiras hipóteses testadas pelas somas de quadrados fornecidas pelos pacotes estatisticos, ocasionando serios problemas tanto aos estatisticos como aos pesquisadores de ciencias aplicadas.

Entre outros, IEMMA (1993), cita alguns dos problemas mais importantes ocasionados pela presença de caselas vazias:

- Podem ocorrer desconexres;

- o teste da interação, em geral,testa que somente algumas interaçơes são nulas;

- As hipóteses sobre médias não ponderadas de linhas e colunas, em geral, têm uma interpretação bastante diferente daquelas com caselas ocupadas

- A interpretação da hipótese depende da posição e do número de caselas vazias;

- Os graus de liberdade da interação devem ser 
34.

escolhidos com cautela.

IEMMA, PALM \& CLAUSTRIAUX (1993) apresemtam uma forma simples para obtenção do projetor associado à hipótese Ho pará qualquer ni vel de desbalanceamento.

Os autores se baseiam na definição de funçơes estimáveis dada por RAO (1845) já apresentada e obtém a soma de quadrados da hipótese através de

$$
\text { SQHo }=y^{\prime} a\left(a^{\prime} a\right)^{-1} a^{\prime} y=y^{\prime} \text { PHo } y
$$

onde $y$ é vetor de observaçßes;

$a$ é o vetor de coeficientes da combinação linear dos componentes do vetor de observaçỡes $y$;

e $a\left(a^{\prime} a\right)^{-1} a^{\prime}=$ PHo e o projetor ortogonal associado a Ho. 
35.

\title{
3. METODOLOGIA
}

\begin{abstract}
Inicialmente serão apresentadas algumas idéias básicas sobre matrizes inversas generalizadas e sobre modelos lineares superparametrizados, de médias de caselas e com restrição $\Sigma$, essenciais para o entendimento da metodologia utilizada neste trabalho.
\end{abstract}

\section{1. MATRI ZES I NVERSAS GENERALI ZADAS}

As matrizes inversas generalizadas são importantes no estudo de modelos lineares, no tocante à estimação. Neste contexto, elas serão usadas exaustivamente nos algoritmos de obtenção das funçశes estimáveis.

Entre outros, serão citados aqui quatro tipos de interesse:

- Inversa Generalizada de Moore-Penrose

$\mathrm{A}^{+}$

- Inversa Generalizada de Mínimos Quadrados

$A^{k}$

- Inversa Generalizada Condicional

$A^{-}$

- Inversa Generalizada GZ

$A^{02}$ 
36.

\section{1.1. I nversa General izada de Moore-Penrose}

Dada uma matriz ${ }_{m} A_{n}$ de posto $k$, a matriz ${ }_{n} A_{m}^{+}$, de posto $k$, que satisfaz as quatro condiçoses seguintes $b$ definida como inversa generalizada de Moore-Penrose de A:
a) $\mathrm{A} \mathrm{A}^{+} \mathrm{A}=\mathrm{A}$
b) $\mathrm{A}^{+} \mathrm{A} \mathrm{A}^{+}=\mathrm{A}^{+}$
c) $\mathrm{AA}^{+}=\left(\mathrm{AA}^{+}\right)^{\prime}$ (simetrica)
d) $A^{+} A=\left(A^{+} A\right)^{\prime}$ (simetrica)

Uma regra para sua obtenção é dada por:

$$
A^{+}=C^{\prime}\left(C C^{\prime}\right)^{-1}\left(B^{\prime} B\right)^{-1} B^{\prime}
$$

(21)

onde B é uma matriz de posto col una completo $\theta$ C é de posto linha completo, tais que $A=B C$.

Pode-se verificar que $A^{+}$assim definida existe sempre é única.

$$
\text { Além disso } \mathrm{AA}^{+} \text {e } \mathrm{A}^{+} \mathrm{A} \text { são simétricas }
$$

i dempotentes.

\subsubsection{I nversa Gemeral izada de Minimos Quadrados}

Dada uma matriz ${ }_{m} A_{n}$ toda matriz ${ }_{n} A_{m}^{l}$ que satistaz as duas condiçres seguintes, e definida como inversa generalizada de mínimos quadrados de A:

a) $A A^{\ell} A=A$

b) $A A^{l}=\left(A A^{l}\right)^{\prime}$ (simetrica $)$

Toda matriz do tipo $A^{l}=\left(A^{\prime} A^{-} A^{\prime} \&\right.$ uma 
inversa de mínimos quadrados de A.

\section{1.3. I nversa Condicional}

Dada uma matriz ${ }_{m}{ }_{n}$, entao toda matriz ${ }_{n} A_{m}^{-}$ tal que $A^{-} A=A$ dita inversa generalizada condicional de A.

Uma regra de obtenção dada pelo Algoritmo de SEARLE (1971).

Dada a matriz ${ }_{m} A_{n}$, de posto K:

a) tomar qual quer matriz não singular, $M$, de ordem $K$;

b) obter $\left(M^{-1}\right)^{\prime}$;

c) substituir em $A$, os elementos de $M$ por seus correspondentes em $\left(M^{-1}\right)^{\prime}$;

d) fazer todos os outros elementos iguais a zero;

e) transpor a matriz resultante;

f) o resultado é uma inversa condicional de A.

\subsubsection{Inversa General izada Ge}

Por ser considerada um caso especial das inversas condicionais, a matriz inversa Ge tambem atende a propriedade $A A^{G 2} A=A$.

A regra para sua obtenção pode ser considerada uma adaptação do algoritmo de SEARLE (1971), dada a seguir.

Para a matriz ${ }_{\mathbf{m}}{ }_{n}$, de posto $K$ : 
38.

a) Obter a matriz $x_{1}$ que e constituida das colunas Iinearmente independentes da matriz $x$, tomadas sequenci al mente;

b) obter $\left(x_{1} \cdot x_{1}\right)^{-1}$;

c) substituir em $x \cdot x$ os elementos de $\left(x_{1}^{\prime} x_{1}\right)$ pelos seus correspondentes em $\left(x_{1}: x_{1}\right)^{-1}$;

d) fazer todos os outros elementos iguais a zero;

e) o resultado obtido é a inversa G2 para a matriz (X'X).

Enquanto a inversa condicional não é única, a

inversa Ge o e.

\section{2. ALgUMaS I DEI AS SOBRE MODELOS LI NEARES}

Para os objetivos deste trabalho serão adotados modelos do tipo

$$
y=x \theta+e
$$

onde:

${ }_{n} y_{1}$ é um vetor de realizaç̧̋es de variáveis al eatórias;

${ }_{n} X_{p}$ e uma matriz conhecida (matriz do delineamentos de posto $K \leq \min \{n, p\}$

$p_{1}$ e um vetor de parametros desconhecidos;

$n_{1} e_{1}$ um vetor de variáveis aleatórias não observáveis, tal que $e \sim\left(\varnothing, I \sigma^{2}\right)$

que é definido como modelo 1 inear de Gaus-Markov, (G.M. 2.

Serão apresentados aqui, algumas das formas assumidas pelo modelo linear G.M. para delineamentos experimentais mais usados no dia a dia dos pesquisadores, 
39.

tanto em situaçâs teóricas como na obtenção dos resultados fornecidos pelos pacotes tradicionais. A escolha das trés formas apresentadas a seguir está relacionada com os objetivos deste trabalho.

Se considerará, conforme IEMMA (1993), o Modelo Superparametrizado (Modelo S) que é excelente para a interpretação da notação RC J, o Modelo de Médias de Caselas CModelo M que pode simplificar a interpretação das hipóteses e o Modelo $\boldsymbol{\Sigma}$ que é essencial para interpretação de certos tipos de somas de quadrados em modelos de posto completo.

\subsubsection{Model o Superparametrizado}

Segundo Searle (1987) um modelo ó dito superparametrizado se ele exibe explicitamente um parámetro para cada efeito dos fatores envolvidos. Será dito Modelo $S$ e denotado por

$$
y=x \theta+\theta \text { como em (2Z) }
$$

Segundo o interesse do pesquisador o Modelo $\mathrm{S}$ pode assumir diferentes caracterizaçőes.

\subsubsection{Equaçzes Normais}

o vetor é um vetor de variáveis aleatórias não observáveis. Sua estimação depende da caracterização 
40.

assumida e da solução do sistema de equaçőes lineares $y=X \theta$, que é inconsistente. Uma solução para ele pode ser obtida através do sistema de equaç̧es normais $X^{\prime} X \theta=X^{\prime} Y$, sempre consistente. Qualquer solução exata, $\theta^{\circ}$, do șistema de equaçб̄es normais $X ' X \theta=$. X'Y $\Leftrightarrow$ solução aproximada de mínimos quadrados para $y=X \theta$ inconsistente. Isto leva ao fato de que, qualquer que seja a solução $\theta^{\circ}$ do sistema de equaçöes normais, 0 vetor $\hat{y}=x e^{\circ}$ invariante, $\theta a$ aproximação de mínimos quadrados para o vetor y das obser vaçơes.

Sendo a matriz $X$ de posto coluna incompleto, então X'X é singular e o sistema de equaçőes normais 6 indeterminado. Suas soluç̧es exatas podem ser obtidas, entre outras, por $\theta^{0}=(X, X)^{6} X^{\prime} Y e$ são soluçðes aproximadas de mínimos quadrados para $y=X \Theta$ inconsistente. Aqui $(X ' X)^{0} \dot{\theta}$ uma inversa generalizada qual quer de $X \cdot X$.

Se $X$. não tem posto coluna completo os componentes do vetor e não são individual mente estimávies.

Segundo RAO", citado por IEMMA (1993), "uma função linear paramétrica $\lambda^{\prime} \theta$ é estimável no modelo linear de Gauss-Markov, se e somente se $\lambda \in C(X ')$ ".

Em termos praticos isto significa que:

a) $E(y)=X \theta \theta$ então $X \theta$ é estimável;

b) combinaços lineares de funçбes estimáveis são também

1RAO, C.R. On the linear combination of observations and the general theory of least squares. Sankhyã, $7\langle 3\rangle$ : 2ap-56, 1945. 
esti máveis.

Além disso, se $\lambda^{\prime} \theta$ é estimável então seu BLUE $\Leftrightarrow$ dado de modo único por $\lambda^{\prime} \theta=\lambda^{\prime} \theta^{\circ}$, onde $\theta^{\circ} \theta$ qualquer sol ução das equaçơes normais. (Teorema de Gauss-Markov).

\subsubsection{Anál ise de Variância}

$\hat{y}=X \theta^{\circ}$ e a aproxi mação de mínimos quadrados para o vetor de observaçơes $y$, invariante para qualquer $\theta^{\circ}$ sol ução das equaçỡes normais.

$$
\hat{y}=x(x \cdot x)^{a} x \cdot y=P y
$$

onde

$$
\left.P=x<x^{\prime} x\right)^{a} x^{\prime}=x x^{+}=x x^{\ell}
$$

* orojetor ortogonal de $y$ sobre o espaço gerado pelas colunas de $X, C(X)$, cuja representação grafica e dada a seguir:

Figura 1. Projeção ortogonal de $y$ sobre $C(x)$.

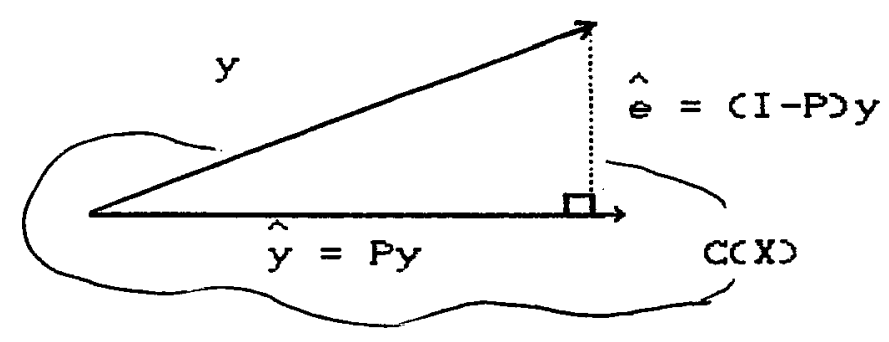

onde

$$
\begin{gathered}
y=\hat{y}+\hat{e} \in \mathbb{R}^{n}, \\
\hat{y} \in C(x), \\
\hat{e} \in C^{\perp}(x)
\end{gathered}
$$


42.

$$
\Leftrightarrow \mathbb{R}^{n}=C(x) \oplus C^{\perp}(x)
$$

$I-P$ o projetor ortogonal de $y$ sobre o complemento ortogonal do espaço coluna de $x, c^{\perp}(X)$.

Dada a ortogonalidade pode-se utilizar o Teorema de Pitágoras e obter a decomposiça ortogonal clássica da análise de variáncia:

$$
\|y\|^{2}=\|\hat{y}\|^{2}+\|\hat{\theta}\|^{2}
$$

onde

$$
\begin{gathered}
\|y\|^{2}=y^{\prime} y=\sum y^{2}=\text { SQ Total } \\
\|\hat{y}\|^{2}=\hat{y^{\prime}} \hat{y}=\sum \hat{y}^{2}=y^{\prime} P y=\theta^{\circ} \cdot x^{\prime} y=\text { SQ Parámetros } \\
\|\hat{\theta}\|^{2}=\hat{\theta}, \hat{\theta}=\sum \hat{\theta}^{2}=y^{\prime}(I-P) y=y^{\prime} y^{\prime} \theta^{\circ}, x \cdot y=\text { SQ Residuo }
\end{gathered}
$$

Por meio de outras partiçäes convenientes

sobre $X$ e $\theta$, pode se obter outras regras para determinar hipóteses sobre funçores lineares parametricas de interesse.

Um procedimento alternativo, útil para estes propósitos $\&$ a obtenção das somas de quadrados das hipóteses. Assim, a soma de quadrados para a hipótese

$$
\text { Ho: } B^{\prime} \theta=\varnothing
$$

onde $B^{\prime} \theta$ é estimável e $B^{\prime}$ tem posto coluna completo é dada, conforme IEMMA (1987), por:

$$
\mathrm{SQHO}=\left(\mathrm{B}^{\prime} \theta^{\circ}\right)^{\prime}\left[\mathrm{B}^{\prime}\left(\mathrm{X}^{\prime} X\right)^{\circ} \mathrm{B}^{-1}\left(\mathrm{~B}^{\prime} \theta^{\circ}\right)\right.
$$

\subsection{3. Notaçåo RC ?}

Tomando-se o modelo dado por (22), a notação RC J 6 definida por

$$
R(\theta)=\theta^{\circ} \cdot x^{\prime} y
$$


43.

onde $\theta^{\circ}$ é uma sol ução para $x \cdot x \theta=x^{\prime} y$.

Se $x$ e $\Theta$ são particionadas da seguinte forma

$\left[\begin{array}{l:l}x_{1} & x_{2}\end{array}\right] e\left[\begin{array}{c}\theta_{1} \\ -\theta_{2}\end{array}\right]$ respectivamente, e se considerarmos

- modelo

$$
y=x_{2} \theta_{2}+e
$$

então

$$
R\left(\theta_{2}\right)=\theta_{2}^{0} \cdot x_{2}^{\prime} y
$$

onde $\theta_{2}^{\circ}$ e uma solução para $x_{2}^{\prime} x_{2} \theta_{2}=x_{2} y$.

Além disso, define-se

$$
R\left(\theta_{1} \mid \theta_{2}\right)=R(\theta)-R\left(\theta_{2}\right)
$$

\subsubsection{Model o de Médias de Casel as}

Os modelos superparametrizados, descritos no Item anterior, são parte integrante da história dos modelos lineares e tem sido de grande valia para os pesquisadores das ciencias aplicadas pois eles mostram explicitamente os parametros $\mu, \alpha, \beta \in \gamma$, sobre os quais estão em geral, os reais anseios de tais pesquisadores. No entanto, eles apresentam alguns incovenientes como, por exemplo, 0 de possuir mais parametros que médias de caselas para estima-los. Este problema pode ser agravado no caso de caselas vazias como ocorre aqui.

Para os propósitos deste trabalho, o modelo de medias apresenta ainda a vantagem de descrever hipóteses 
44.

com grande simplicidade, em relação ao Modelo $\mathrm{S}$.

Segundo SPEED, HOCKING \& HACKNEY (1978), derine-se o Modelo de Medias de Caselas ou Modelo M como o model o linear

$$
y=w+e
$$

onde:

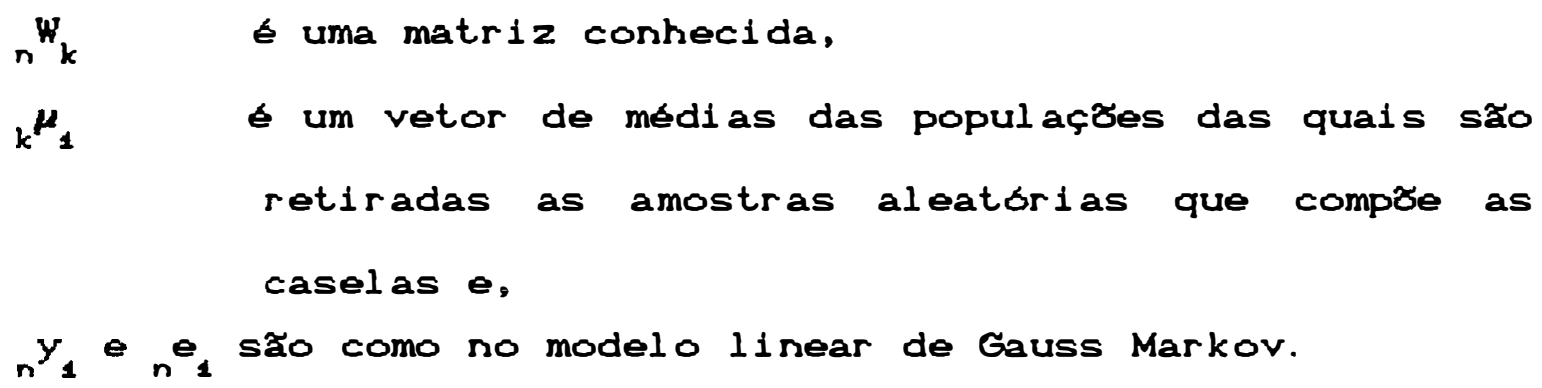

\subsubsection{Equaçós Normais}

O sistema de equaçชes normais $W^{\prime} W_{\mu}=W^{\prime} y \in$ em geral, muito mais simples que $X^{\prime} X \theta=x^{\prime} y$ do Modelo $S$. Se $W$ tem posto coluna completo, $W \cdot W$ e positiva definida e o sistema tem solução única. Neste contexto, a solução exata $\hat{\mu}=(W \cdot W)^{-1} W^{\prime} y$, que $e$ solução aproximada para o sistema incosistente $y=W \mu$, coincide com 0 estimador de minimos quadrados para $\mu$. Assim, o BLUE de $\mu_{i j}$ e dado por $\hat{\mu}_{i j}=\bar{y}_{i j}$, isto e, a media da casela $(1, j)$.

Natur al mente

$$
\hat{\lambda} \cdot \mu=\lambda \cdot \hat{\mu} \quad \text { (Teorema Gauss-Markov) }
$$


45.

\subsubsection{Anallse de Vartáncla}

A aproximação de minimos quadrados para o vetor de observaçzes $y$ é dado por $\hat{y}=\hat{\mu}=X \theta^{0}$.

os outros aspectos da análise de variáncia são como no Modelo $\mathrm{S}$.

\section{2. 3. Nodelo com Restriçâo Paramstrica do Tipo $\Sigma$}

o Modelo $\Sigma$ \& um modelo linear superparametrizado, com restriçశes paramétricas do tipo "soma igual a zero". Sera representado por:

$$
y=z \dot{\theta}+e
$$

\subsubsection{Equaçöes Mormals}

De modo análogo aos modelos precedentes temos

$$
z \cdot \dot{\theta}=z \cdot y
$$

com solução única, pois $\boldsymbol{Z}$ tem posto coluna completo.

Assim,

$$
\dot{\theta}^{\circ}=\left(z \cdot z^{-1} z^{\prime} y\right.
$$

\section{2. 3.2. Anál 1se de Vartância}

Para os objetivos deste trabalho, a importancia dó Modelo $\boldsymbol{\Sigma}$ está na obtenção da soma de a. quadrados para um parametro ajustado para todos os outros, 
46.

por exemplo, considerando um modelo com dois fatores e inter ação, tem-se:

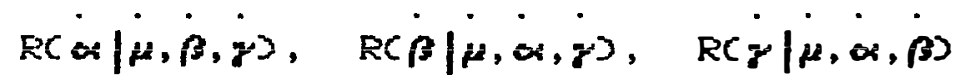

em lugar do ajuste sequencial.

Para este caso,será aplicado aqui o processo da inversa de parte da inversa, de SEARLE (1971) que facilita a obtençăo das somas de quadrados ajustadas.

\section{3. ALGUMAS I DEI AS SOBRE ESTI MAÇAO}

- pacote SAS, segundo o Manual do SAS, capitulo $\theta$ (1900), se baseia em alguns conceitos de estimabilidade para construir os quatro tipos de funçชes estimáveis oferecidas pelo seu procedimento GLM.

Partindo-se de model os 1 ineares, tais como

$$
y=X \theta+e, j a ́ \text { apresentados em (Z2) }
$$

que t\&m $E(y)=X \theta$, o objetivo inicial estimar os elementos de ou alguma combi nação 1 inear de seus elementos.

Isto feito calculando-se combinaçres lineares dos componentes do vetor de observaçбes y. Para estimar-se uma combi naçăo linear dos componentes do vetor $\theta$, Le, deve-se ser capaz de encontrar uma combi nação li near dos componentes do vetor $y$ que tem como valor esperado Le. Tem-se portanto, a seguinte definiçăo:

Lo estimável se e somente se existe uma combinação linear dos componentes do vetor y cujo valor esperado e Le.

Qualquer combinaçăo linear das observaçชes, 
Y, por exemplo, $\mathrm{KY}$, tera como valor esperado, $\mathrm{E}(\mathrm{KY})=\mathrm{KX} \boldsymbol{\theta}$. Assim, o valor esperado de qualquer combinação 1 inear dos componentes do vetor $y$ é igual a mesma combinação linear das linhas de $\mathrm{X}$ multiplicadas por $\theta$.

Portanto:

Le estimável se e somente se uma combinação 1 inear das linhas de $X$ que 6 igual a $L$ pode ser encontrada.

Assim, as linhas de $X$ formam um conjunto gerador do qual um $L$ pode ser construido. Como $x$ pode ser construída das linhas de $\mathrm{X} \cdot \mathrm{X}$, isto $6, \mathrm{X}=\left[\mathrm{X}(\mathrm{X}, \mathrm{X})^{-}(\mathrm{X} \cdot \mathrm{X})\right]$, as linhas de $X \cdot X$ formam tamberm um conjunto gerador do qual funçỡes estimáveis $L$ podem ser construídas. Similarmente as linhas de $(X \cdot X)^{-} X \cdot X$ formam tambem um conjunto gerador para L.

Portanto, se L \& gerado como uma combinaçăo linear de $x, x^{\prime} x$ ou $(x \cdot x)^{-} x \cdot x$, Le estimável.

Uma vez que um $L$, de posto linha completo tenha sido formado de um conjunto gerador, Le pode ser estimado por $L \theta^{\circ}$, onde $\theta^{\circ}=\left(X^{\prime} X\right)^{-} X^{\prime} y$. Da teoria geral de modelos lineares, $L^{\circ} \in$ o melhor estimador imparcial de Le.

Com muitos conjuntos geradores disponi vels a questão e: qual o melhor para representar L? Naturalmente, um conjunto gerador contendo o minimo de linhas cde posto Iinha completoj e um máximo de elementos nulos e desejável.

Por 1sso, a PROC GLM opta por calcular uma inversa generalizada (Ga) de $X^{\prime} X$ tal que $\left(X^{\prime} X\right)^{\mathbf{d 2}} X^{\prime} X$ contem numerosos zeros e que suas linhas não nulas são linearmente 
48.

independentes. 0 GLM usa as linhas não nulas de $\left(X^{\prime} X\right)^{a 2} X^{\prime} X$ para representar $\mathrm{L}$.

\section{4. OS QUATRO TI POS DE FUNÇOES ESTI MAVEI S}

Será apresentado aqui um resumo das
informaçóes que o Manual do SAS, Capitulo 9 (1990).
fornece sobre cada tipo de funçơes estimáveis de que a
PROC GLM dispơe que servirá de base para o estudo
presente.

\subsubsection{Forma Geral de Fumçס̄es Estimávels}

- PROC GLM constról uma forma geral para as funçớes estímáveis através de $(X \cdot X)^{02} X \cdot X$ usando suas 1 inhas não nulas para representar L.

A forma geral de funçzes estimáveis envolve todos os parametros do modelo e a cada linha não nula, linearmente independente, de $(X \cdot X)^{62} X^{\prime} X$ e associado um símbolo L. O número de símbolos L representa o posto máximo da hipótese associada.

\section{4, 2. Funçठes Estimáveis do Tipo I}

As SQ tipo I são dependentes da ordem do modelo, cada efeito é ajustado somente para os efeitos: precedentes no modelo. 
49.

As funçชes estimáveis do tipo I săo obtidas fazendo-se a devida partição na matriz $x$, de acordo com os parametros envolvidos no modelo, e com a ordem em que são apresentados e calcul ando-se:

$$
\begin{aligned}
& G_{1}=\left(x: x_{1}\left|x: x_{2}\right| x: x_{9}\right) \\
& G_{2}=c \theta\left|X_{2}^{\prime} M_{1} X_{2}\right| X_{2}^{\prime} M_{1} X_{9}^{2} \\
& \left.G_{3}=C \theta|\otimes| X_{3} M_{2} X_{3}\right) \\
& M_{1}=I-x_{1}\left(x_{1}^{0} x_{1}\right)^{-} x_{1}^{\prime} \\
& M_{2}=M_{1}-M_{1} X_{2} C X_{2}^{\prime} M_{1} X_{2}{ }^{-} X_{2}^{\prime} M_{1}
\end{aligned}
$$

No procedimento GLM, as funçớes estimáveis tipo I, impressas quando a opção $E_{1}$ e requerida são:

$$
\begin{aligned}
& G_{1}^{*}=\left(x: x_{1}\right)^{-} G_{1} \\
& G_{2}^{*}=\left(X_{2}^{*} M_{1} X_{2}\right)^{-} G_{2} \\
& G_{9}^{*}=\left(X_{9}^{\prime} M_{2} X_{9}\right)^{-} G_{9} \\
& G_{1}^{*}, G_{2}^{*} \text { e } G_{9}^{*} \text { geram funçชes estimáveis do tipo }
\end{aligned}
$$

I. Atribuindo-se valores arbitrários aos simbolos $L$ que aparecem nas funçశes estimáveis, obtem-se hipóteses tipo I.

$$
\text { As hipoteses tipo I são consideradas }
$$

adequadas para os seguintes modelos:

- modelos de análise de variancia balanceados especificados em sequéncia própria cpor exemplo, interação não precedendo efeitos principaiss;

- modelos hierárquicos cespecificados em sequéncia apropriadal;

- modelos de regressão poli nomial. 
క50.

\section{4, 3. Furç8es Estimáveis Tipo II}

As $S Q$ tipo II correspondem à notação $R C J$ em que cada efeito é ajustado para todos os outros possiveis.

Para efeitos principais, digamos, $C A, B$ e $C$ come variáveis de classificaçăos, as SQ tipo II representadas pela notação RC J estăo apresentadas na Tabela 2.

Tabela 2 - Somas de Quadrados Tipo II em Termos da Notação $\mathrm{RC} 3$

\begin{tabular}{cl}
\hline Efeito & SQ Tipo II \\
\hline$A$ & $R(A \mid B, C)$ \\
$B$ & $R(B \mid A, C)$ \\
$C$ & $R C C \mid A, B)$ \\
\hline
\end{tabular}

Para modelos que envol vam interaçơes cque serão considerados neste trabal hos, não possivel obter um teste de hipóteses para um efeito principal livre de parametros de efeito do nivel superior com os quais o efeito está envol vido.

As funçžes estimáveis tipo II para um efeito F tem um L da seguinte forma:

a) todas as colunas de $L$ associadas com efeitos não contendo $F_{1}$, (exceto $F_{1}$, serão formadas por zeros;

b) a submatriz de $L$ associada com o efeito $F_{1}$ será: $\left(X_{1} \cdot x_{1}\right)^{-}\left(x_{1} \cdot M x_{1}\right)$

onde 
51.

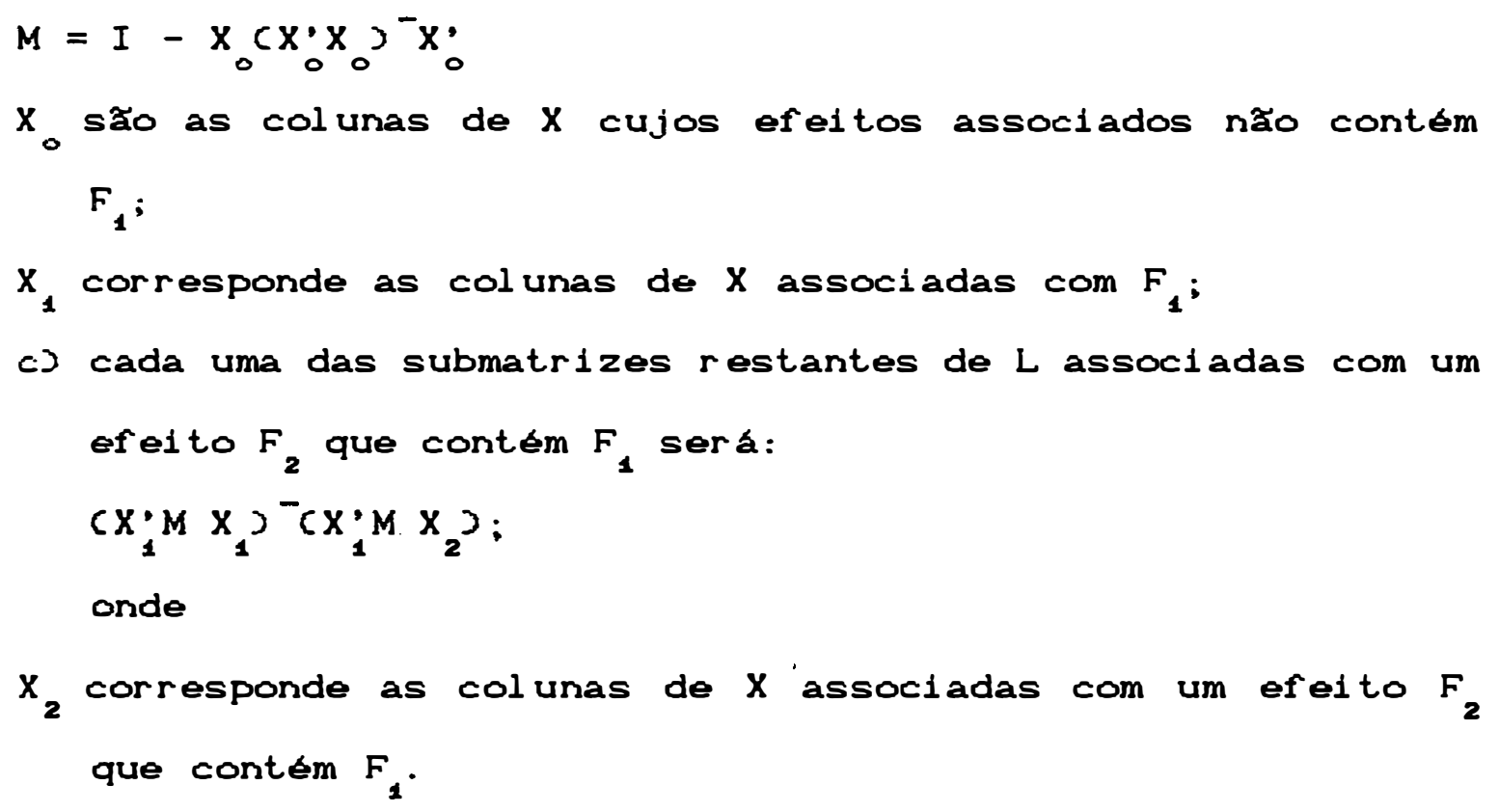

\section{4. 4. Funçōes Estimáveis Tipo III}

A filosofia que norteia as hipoteses tipos III e IV está baseada no fato de que os testes de hipóteses feitos para qualquer efeito seriam os mesmos para todos os : del ineamentos com a mesma forma geral de funçớes estimáveis. 
Se.

- SAS usa o Modelo $\Sigma$, apresentado anteriormente, para obter as funçชes estimáveis tipo III, ajustando os parkmetros deste modelo para todos os demais.

As funçбes estimaveis tipo III são construidas trabalhando-se com a forma geral de funçชes estimáveis. Os seguintes passos são considerados na obtenção de uma função para um efeito $F_{1}$ :

a) para cada efeito no modelo, exceto $F_{1}$ e aqueles efeitos que contem $F_{1}$, igualar os coeficientes na forma geral de funçбes estimáveis a zero. Se $F_{1}$ não está contido em qualquer outro efeito, este passo define a hipotese tipo III. Se $F_{1}$ esta contido em outro efeito, seguir para o segundo passo;

b) se necessario, criar simbolos novos e iguala-los a expressão composta no bloco $F_{1}$ a fim de obter a forma mais simples para os coeficientes de $F_{1}$;

c) igualar todos os coeficientes simbólicos fora do bloco $F_{1}$ à uma função linear dos simbolos no bloco $F_{1}$ a fim de fazer a hipotese de $F_{1}$ ortogonal as hipoteses associadas com os efeitos que contem $F_{1}$.

\subsubsection{Fumç8es Estimáveis do Tipo IV}

As funçชes estimáveis tipo IV são similares as do tipo III, quando não existem caselas vazias. Elas não são construidas com base na notaçăo RC J, simplesmente consideram um subconjunto dos dados onde não ocorrem casel as 
53.

vazias.

$$
\text { Se existem caselas vazias, as } S Q \text { tipo IV }
$$

podem não ser únicas poís dependem da posição e do número de caselas vazias.

De um modo geral, pode-se interpretar as hipóteses tipo IV como hipóteses sobre contrastes entre medias de casel as que estão na mesma col una (ou linha).

- concelto básico para se construir a hipotese tipo IV é que os coeficientes de qualquer efeito, $F_{1}$ por exemplo, são distribuidos equitativamente através dos efeitos de nivel superior que contém $F_{1}$. Quando ocorrem caselas vazias, esta mesma ideia deve ser seguida, tomando-se cuidado com a forma como o concelto distributivo e aplicado.

A construção de funçớes estimáveis tipo IV começa com a construção das hipóteses tipo III. Isto é, para um efeito $F_{1}$, igualar a zero todos os coeficientes na forma geral que não pertencem a $F_{1}$ ou a qualquer outro efeito contendo $F_{1}$. Se $F_{1}$ não está contido em qualquer outro efeito, então as funçres tipo IV $c e$ tipos II e IIIJ terá sido encontrada. Se $F_{1}$ está contido em outros efeitos então distribuir os coeficientes associados a $F_{1}$ entre os coeficientes fora do bloco $F_{1}$.

Neste contexto, conforme será visto adiante, as somas de quadrados resultantes tem as seguintes caracteristicas:

a) se os dados săo bal anceados: 
54.

SQ Tipo I = SQ Tipo II = SQ Tipo III = SQ Tipo IV

b) Se todas as caselsa são ocupadas:

SQ Típo III = SQ Típo IV

c) se o modelo não tem interação:

SQ Tipo II = SQ Tipo III = SQ Tipo IV

\subsection{HI POTESES MAIS GOMUNS SOBRE LI MHAS, COLUNAS $E$ I NTERAÇATO}

Aqui, serão apresentadas as hipóteses sobre linhas, colunas e interação decorrentes das funçỡes estimáveis fornecidas pelo SAS. Será tratado aqui um modelo com interação e caselas vazias pois a sua presença complica a estruturação das hipóteses.

Estas hipóteses serão apresentadas em termos do Modelo $M$ para efeito de simplificação e baseadas na estrutura de dados apresentados na Tabela 3, com duas linhas, tres colunas e a casela correspondente à $\mu_{2 g}$ vazia.

Tabel a 3 - Número de Observaçớs Referentes a Cada Média de Casela

\begin{tabular}{lll}
\hline$\mu_{11}(2)$ & $\mu_{12}(1)$ & $\mu_{13}(2)$ \\
$\mu_{21}(2)$ & $\mu_{22}(3)$ & -
\end{tabular}


55.

\subsubsection{Hipóteses Sobre a Interaçảo}

Como é apresentado por IEMAA (1993), se não temos caselas vazias, entåo, para "a" niveis do fator A (linhas) e "b" niveis do fator B (colunas), tem-se $1 / 4 a b(a-1)(b-1)$ interaçơes possiveis, entre as quais $(a-1)(b-1)$ são 1 inearmente independentes. Agora, quando existem caselas vazias, existem menos que $1 / 4$ ab(a-1)(b-1) interaçбes que podem ser estimadas e, pode ocorrer que menos de $(a-1)(b-1)$ sejam linearmente independentes, modificando o número de graus de liberdade da interaçăo.

Considerando-se 0 modelo $y_{i, j}=\mu_{i j}+e_{i j k}$, cada componente da interação é dado por

$$
\Delta_{i j, i^{\prime} j^{\prime}}=\mu_{i j}-\mu_{i^{\prime} j}-\mu_{i j^{\prime}}+\mu_{i^{\prime} j^{\prime}}
$$

Assim, se não há caselas vazias, com a=Z e $b=3$, existem 3 interaçбes estimáveis $c \Delta$ entre as quais duas são linearmente independentes. Para o caso do exemplo estas regras não se aplicam, pois há uma casela vazia. A média $\boldsymbol{\mu}_{29}$ não é estimável no modelo com interação e a única interação estimável tem seu BLUE dado por

$$
\overbrace{\mu_{11}-\mu_{12}-\mu_{21}+\mu_{22}}=\bar{y}_{11 .}-\bar{y}_{12 .}-\bar{y}_{21}+\bar{y}_{22} \text {. }
$$

levando ao fato de que, nesse caso, existe só um grau de liberdade para interaçăo. Observa-se aqui uma diferença fundamental entre o caso com todas as caselas ocupadas e o caso com caselas vazias: no primeiro a hipótese sobre interação testa se todas as interaçశes săo nulas, no segundo 
56.

testa se algumas determinadas interaçชes são nulas.

Segundo IEMMA (1993), se existem caselas yazias, o teste para interação não deve ser um criterio para escolha do modelo com ou sem interaçăo. Esta escolha podera ser baseada, digamos, apenas na experiencia do pesquisador.

$$
\begin{aligned}
& \text { Neste caso, tem-se } \\
& \mathrm{Ho}^{(7)}: \boldsymbol{\mu}_{11}-\boldsymbol{\mu}_{12}-\boldsymbol{\mu}_{21}+\boldsymbol{\mu}_{22}=0
\end{aligned}
$$

que é diferente da hipótese de que todas as interaçశes sejam nulas. Seŕa visto adiante que

$$
\dot{S Q H}{ }^{(p)}=R(\gamma \mid \mu, \alpha, \beta)=R(\dot{\gamma} \mid \dot{\mu}, \dot{\alpha}, \dot{\beta})
$$

\subsubsection{Hipóteses Mais Comms Sobre Efeitos de \\ Li nhas}

Serão descritos agora, quatro tipos de hipóteses sobre efeitos de 1 i nhas para a estrutura de dados apresentada.

\subsubsection{Hipoteses Sobre Medias Ponderadas de Linhas}

Considerando o modelo de medias de caselas e - exemplo apresentado, uma hipótese sobre medias ponderadas de linhas é dada por:

$$
H_{0}{ }^{(1)}: \frac{2 \mu_{11}+\mu_{12}+2 \mu_{19}}{5}=\frac{2 \mu_{21}+3 \mu_{22}}{5}
$$


57.

A hipótese sobre médias ponderadas de linhas equivale a hipotese tipo I apresentada no 1 tem 3.4 .2 e em termos da Notação RC Y sua soma de quadrados equi vale à

$$
S Q H_{0}(1)=R(\alpha \mid \mu)
$$

\subsection{2. Hi póteses Sobre Médias Ponderadas de Linhas Ajustadas para Col unas}

$$
\begin{aligned}
& \text { A forma geral da hipotese o do tipo: } \\
& H_{0}^{(2)}: \sum_{i} n_{i j} \mu_{i j}=\sum_{i}, \sum_{j} \frac{n_{i j} n_{i, j} \mu_{i, j}}{n_{\cdot j}}
\end{aligned}
$$

que é de dificil interpretação.

Um modo prático para descrevella pode ser obtido usando $\mathrm{Ho}^{\langle 2\rangle}$, da seguinte forma:

a) tomar os "lados esquerdos" de $\mathrm{Ho}_{0}$ "l) como os "lados esquerdos" da nova hipótese;

b) os "lados direitos" da expressão podem ser obtidos substituindo-se na expressão do lado esquerdo as médias das caselas $\mu_{i j}$ pelas respectivas medias ponderadas de colunas. Assim, as duas hipóteses equivalentes para o modelo em questão são:

$$
H_{0}{ }^{(2)}: \frac{2 \mu_{11}+\mu_{12}+2 \mu_{19}}{5}=\frac{2 \bar{c}_{1}+\bar{c}_{2}+2 \bar{c}_{9}}{5}
$$

ou de forma equi valente 
58.

$$
H_{0}^{(2)}: \frac{2 \mu_{11}+3 \mu_{22}}{5}=\frac{2 \bar{c}_{1}+3 \bar{c}_{2}}{5}
$$

onde

$$
\bar{c}_{1}=\frac{2 \mu_{11}+2 \mu_{21}}{4} ; \quad \bar{c}_{2}=\frac{\mu_{12}+3 \mu_{22}}{4} ; \quad \bar{c}_{9}=\mu_{19}
$$

săo as medias ponderadas das col unas.

A hipotese sobre médias ponderadas de linhas ajustadas para colunas equivale à hipótese tipo II, apresentada no item 3.4 .3 em termos da notaçăo $R(J$, tem-se

$$
\left.S Q H_{0}(2)=R C \alpha \mid \mu, \beta\right)
$$

\subsubsection{Hipoteses Sobre Medias Máo Pondera- das de Linhas}

Neste caso, a ocorréncia de caselas vazias ira interferir na interpretação das hipoteses que estao sendo testadas com base em $R(\dot{\alpha} \mid \dot{\mu}, \dot{\beta}, \dot{\gamma})$, pois esta hipotese e equi valente a tipo III apresentada no 1 tem 3.4.4.

A hipótese sobre medias não ponderadas de linhas, para o exemplo em questão, $\&$ a seguinte:

$$
H_{0}^{(9)}: \frac{\mu_{11}+\mu_{12}}{2}=\frac{\mu_{21}+\mu_{22}}{2}
$$


59.

a soma de quadrados dada por $R(\dot{\alpha} \mid \dot{\mu}, \dot{\beta}, \dot{\gamma})$ se refere a uma hipótese sobre medias ponderadas de linhas "nas colunas compl etas".

3.5.2. 4. Hi póteses Sobre Médias Máo
Ponderadas de Linhas, Segundo as
Gaselas Vazias

Săo hipóteses sobre contrastes entre médias de caselas que estão na mesma coluna, começando pela última linha. Correspondem as hipóteses tipo IV fornecidas pelo SAS.

Para o exemplo em questão, uma hipótese deste tipo é dada por

$$
H_{0}^{(\theta)}: \mu_{21}+\mu_{22}=\mu_{11}+\mu_{12}
$$

Se alterarmos a posição das linhas em que estão dispostos os dados, se irá obter outra hipotese.

Além disso, a soma de quadrados devido a $H_{0}^{(\theta)}$ não tem relação com a notação $R C$ I como as demais.

\subsubsection{Hipóteses Mais Comms Sobre Efeitos de Col unas}

Tendo em vista ainda a estrutura de dados do exemplo e o Modelo M, serão apresentadas as hipóteses mais comuns sobre efeitos de colunas. 
60.

\subsubsection{Hipoteses Sobre Medias Ponderadas de Col unas}

Para colunas, a hipótese será composta por duas linhas, pois tem-se tres colunas a comparar e portanto, dois graus de liberdade. Para construção desta hipótese, se irá escolher duas médias ponderadas de colunas entre as trés possiveis do exemplo. Desse modo, ela poderáa ter aparentemente várias formas, que são, no entanto, equi val entes.

$$
\begin{aligned}
& \text { Para o exemplo em questão, busca-se testar } \\
& H^{(4)}: \frac{2 \mu_{11}+2 \mu_{21}}{4}=\frac{\mu_{12}+3 \mu_{22}}{4}=\mu_{19}
\end{aligned}
$$

Uma hipotese sobre medias ponderadas de colunas pode ser obtida comparando-se a primeira coluna com as outras duas, da seguinte forma

$$
H_{0}(\Delta):\left\{\begin{array}{l}
\frac{2 \mu_{11}+2 \mu_{21}}{4}=\frac{\mu_{12}+3 \mu_{22}}{4} \\
\frac{2 \mu_{11}+2 \mu_{21}}{4}=\mu_{19}
\end{array}\right.
$$

Em termos da notação RC 2, sua soma de quadrados a dada por

$$
\left.S Q H_{0}^{(4)}=R C \beta \mid \mu\right)
$$


61.

3. 5. 3.2. Hipótese Sobre Médias Ponderadas de Col umas Ajustadas para Linhas

$$
\begin{aligned}
& \text { Seja, entre outras equival entes: } \\
& \qquad \begin{array}{c}
\frac{2 \mu_{11}+2 \mu_{21}}{4}=\frac{2 \bar{l}_{1}+2 \bar{l}_{2}}{4} \\
\frac{\mu_{12}+3 \mu_{22}}{4}=\frac{-l_{1}+3 \overline{l_{2}}}{4}
\end{array}
\end{aligned}
$$

onde:

$$
\bar{l}_{1}=\frac{2 \mu_{11}+\mu_{12}+2 \mu_{19}}{5} \quad e \quad \bar{l}_{2}=\frac{2 \mu_{21}+3 \mu_{22}}{5}
$$

Esta hipotese se equivale a hipotese tipo II fornecida pelo SAS e sua soma de quadrados corresponde, em termos da Notação RC J a:

$$
S Q H_{0}^{(S)}=R(\beta \mid \mu, \infty)
$$

\subsubsection{Hipótese Sobre Médias Máo Ponderadas de Col unas}

Aqui, como no caso das linhas, há problemas quando existem caselas vazias.

Para o exemplo em questão, uma hipotese com dois graus de 1 iberdade e: 
62.

$$
H_{0}^{(0)}:\left\{\begin{array}{l}
\frac{\mu_{11}+\mu_{21}}{2}=\frac{\mu_{12}+\mu_{22}}{2} \\
\frac{\mu_{11}+\mu_{12}}{2}=\mu_{19}
\end{array}\right.
$$

Esta hipótese corresponde a hipótese tipo III para colunas e portanto, conforme o 1 tem 3.4.4:

$$
S Q H_{0}^{(\sigma)}=R(\dot{\beta} \mid \dot{\mu}, \dot{\alpha}, \dot{\gamma})
$$

\subsubsection{Mipbteses Sobre Medias Má Ponderadas de Col unas, Segundo as Casel as Vazias}

São hipóteses sobre contrastes entre médias de caselas que estão na mesma linha, começando pela última col una.

Para o exemplo em questao, uma hipótese deste tipo o dada por

$$
H_{0}^{(9)}:\left\{\begin{array}{l}
\mu_{19}=\mu_{12} \\
\mu_{19}=\mu_{11}
\end{array}\right.
$$

Da mesma forma que para $H_{0}^{(\theta)}$, a soma de quadrados devido a $H_{0}^{(D)}$ nå tem relaçao com a notação $R C$.

3.6. CONENTARI OS ADI GI ONAIS SOBRE AS FUNÇOES ESTI MAVEIS

\section{E HI POTESES TI PO I I}

Considera-se que o objetivo deste trabal ho é 
63.

mostrar como o SAS constroi os quatro tipos de funçBes estimáveis fornecidas pelo PROC GLM.

Dentre todas, a obtenção das funçōes estimáveis tipo III parece ser a mais confusa. Assim se justifica a apresentação aqui de formas mais práticas e compreensiveis de obte-las e interpreta-las que aquela apresentada pelo SAS.

\subsubsection{Outra Forma de Obtençăo das Funçōes Estimá- veis Tipo III}

SEARLE (1980) apresenta uma forma prática para obtençăo das funçชes esti máveis tipo III para um modelo com interação e algumas caselas vazias, atraves de um - exemplo com dois niveis do fator $A$, trés nivels do fator $B$ e - número de observaçzes por casela é apresentado na Tabela 4.

Tabel a 4 - Número de ObservaçÕes por Casela

\begin{tabular}{lllll}
\hline & \multicolumn{4}{c}{$j$} \\
& 1 & 2 & 3 & $n_{i}$ \\
\hline 1 & 3 & 2 & 1 & 6 \\
2 & 2 & 2 & 0 & 4 \\
\hline$n . j$ & 5 & 4 & 1 & 10 \\
\hline
\end{tabular}

As funçros esti máveis (f) a serem construídas para col unas são combinaçชes lineares dos parămetros e 
64.

a) são contrastes baseados em B;

b) não envol vem os $\alpha e$

C) são ortogonais aos contrastes dos $\gamma$ que explicam $R C \gamma \mid \mu, \alpha, \beta)$

Um exemplo de contrastes convenientes satisfazendo $(a)$ e (b) é:

$\beta_{1}-\beta_{2}+1 / 2\left(\gamma_{11}+\gamma_{21}\right)^{2}-1 / 2\left(\gamma_{12}+\gamma_{22}{ }^{2}\right.$

$\beta_{1}-\beta_{9}+\gamma_{11}-\gamma_{19}$

$\beta_{2}-\beta_{9}+\gamma_{12}-\gamma_{19}$

Tomando-se combi naçชes lineares deles, vem:

$\left.\left.f=m_{1}\left[\beta_{1}-\beta_{2}+1 / 2 \gamma_{11}+\gamma_{21}\right]-1 / 2 \gamma_{12}+\gamma_{22}\right]\right]+$

$+m_{2}\left[\beta_{1}-\beta_{9}+\gamma_{11}-\gamma_{19}\right]+m_{9}\left[\beta_{2}-\beta_{9}+\gamma_{12}-\gamma_{19}\right]$

Agora, usando (c) o único contraste disponivel para compor $R C \gamma \mid \mu, \alpha, \beta)$ e $\gamma_{11}-\gamma_{12}-\gamma_{21}+\gamma_{22}$ Os coeficientes dos parametros neste e em f são apresentados na Tabel a 5. 
65.

Tabela 5 - Coeficientes m para a Combinaçăo Linear $f$ e Para a Interação

\begin{tabular}{|c|c|c|c|c|}
\hline \multirow[b]{3}{*}{ contraste } & \multicolumn{3}{|c|}{$\beta$} & \\
\hline & $\beta_{1}$ & $\beta_{2}$ & $\beta_{B}$ & \\
\hline & $m_{1}+m_{2}$ & $\begin{array}{c}-m_{1}+m_{9} \\
0\end{array}$ & $m_{0}^{-m_{2}-m_{9}}$ & \\
\hline & & $\gamma$ & & \\
\hline & $\gamma_{19}$ & $\gamma_{21}$ & $\gamma_{22}$ & \\
\hline $\begin{array}{l}f \\
\text { contraste } \gamma\end{array}$ & $\begin{array}{c}-m_{2}-m_{3} \\
0\end{array}$ & $\begin{array}{cc}1 / 2 & m_{1} \\
-1 & \end{array}$ & $\begin{array}{c}-1 / 2 m \\
1\end{array}$ & $m_{1}$ \\
\hline
\end{tabular}

A ortogonalidade entre os dols $1 \mathrm{mplica}$

$$
\begin{gathered}
\text { 1/2 } m_{1}+m_{2}+1 / 2 m_{1}-m_{9}-1 / 2 m_{1}-1 \Omega m_{1}=0, \text { isto } 6, \\
m_{2}=m_{3}
\end{gathered}
$$

Os graus de liberdade para o fator $B$ săo dois, portanto, existem dois valores arbitrálos para os coeficientes $m$. Considera-se que a parte de $f$ para $\beta_{1} \in \beta_{2}$ $L_{1} \beta_{1}+L_{5} \beta_{2}$, isto $6:$

$$
\begin{aligned}
& \quad m_{1}+m_{2}=L_{4} \text { e }-m_{1}+m_{9}=L_{5} \\
& \text { Portanto, } \\
& m_{1}=1 / 2\left(L_{4}-L_{5}\right) \text { e } m_{2}=m_{9}=1-2\left(L_{4}+L_{5}\right)
\end{aligned}
$$

obtendo-se: 
66.

$$
\begin{aligned}
f= & L_{4} \beta_{1}+L_{5} \beta_{2}-\left[L_{4}+L_{5}\right] \beta_{5}+1 / 4\left[3 L_{4}+L_{5}\right] \gamma_{11}+ \\
& +1 / 4\left[L_{4}+3 L_{5}\right] \gamma_{12}-\left[L_{4}+L_{5}\right] \gamma_{19}+1 / 4\left[L_{4}-L_{5}\right] \gamma_{22}- \\
& -\left[L_{4}-L_{5}\right] \gamma_{22}
\end{aligned}
$$

que equivale as funçôes estimáveis do tipo III obtidas atraves da metodologia indicada pelo Manual do SAS, porém construidas de forma mais simples.

\section{6. 2. Un Algoritmo para Transformar as Hipoteses em Termos do Modelo $\Sigma$ em Hipoteses do Model o $S$ Quando Ocorrem Casel as Vazias}

As hipoteses tipo III são obtidas com base no Modelo $\Sigma$. Assim, as hipóteses sobre linhas e colunas são da seguinte forma:

$$
H: \dot{\alpha}_{i}=0 \forall i \quad H: \dot{\beta}_{j}=0 \forall \mathrm{J}
$$

onde $\dot{\alpha}_{i} \in \dot{\beta}_{j}$ săo os parámetros do modelo restrito, como em 3. 2. 3 .

Por 1sso, as hipóteses que são aparentemente simples em termos dos parametros do modelo restrito são complexas quando colocadas em termos dos parámetros do modelo irrestrito. Para converter a hipotese em termos dos parametros do modelo irrestrito, quando ocorrem caselas vazias, SEARLE (1987) apresenta o seguinte algoritmo.

Seja "s" o número de caselas completas, $\beta$ vetor de parametros no modelo irrestrito e $\beta$. (de ordem s) 0 vetor de parametros do modelo restrito, de posto completo. 
67.

Se $x \in X_{\text {e }}$ sao definidas por

$$
E(y)=X \beta E E(y)=x^{\prime} \beta^{\prime}
$$

entzo define-se $w$ e w como as matrizes formadas pelas $s$ linhas distintas de $x \in x_{\text {a }}$ respectivamente. Se $\beta \in \beta_{\text {s }}$ sao rel acionadas atraves da equação

$$
W_{\mathrm{g}} \beta_{\mathrm{g}}=W \beta
$$

- We posto completo, entao

$$
\beta_{0}=w^{-1} W
$$

e os parametros do modelo restrito (Modelo $\sum$ estao dados em termos do modelo irrestrito (Modelo SJ.

\subsection{PROCEDI NENTO}

As regras e conceltos apresentados ate aqui, serăo aplicados a dois exemplos. Os exemplos se constituem de experimentos com dols fatores, interaçăo, número diferente de observaçбes por caselas e caselas vazias.

- primeiro exemplo apresenta uma estrutura mals simples com uma única casela vazia. o objetivo deste exemplo e introduzir, de forma mals compreensivel, o que está por trás de cada tipo de soma de quadrados, fatos que o usuário multas vezes desconhece.

o segundo exemplo, com mai or número de ni vels - caselas vazias, tem o objetivo de demonstrar a complexidade que as hipoteses assumem neste caso.

Os dados apresentados são ficticios e o número de observaçбes por casela é sempre pequeno, com 
68.

vistas a facilitar a compreensão dos procedimentos, uma vez que o tamanho do experimento não interfere nos objetivos deste trabalho.

Os dois casos apresentados aqui poderăo ser adaptados a situação do usuário e com isto ele terá condiçres de interpreta-1a. Este fato se tornaria extremamente complexo caso ele só dispusesse do Manual do SAS, pois este contem apenas consideraçனes gerais sobre os tipos de funçช̃es com poucos exemplos.

\section{8. O SI STEKA SAS}

As funçбes estimáveis construidas aqui passo a passo, são apresentadas pelo PROC GLM do SAS quando as opçбes $S S$ e $E$ do comando MODEL são requeridas após a especificação do modelo, na seguinte forma:

model $y=A B A * B, S S 1$ SS2 SS3 SS4 E E1 E2 E3 E4 XPX I;

As opçбes SS1, SSZ, SS3 e SS4 proveem as somas de quadrados tipos I, II, III e IV respectivamente.

A opção $E$ prove a forma geral de funçơes estimáveis e E1, E2, E3 e E4 as funçชes estimáveis tipos I, II, III e IV.

As opçชes XPX e I fornecem a matriz X'X e a inversa Ge para a matriz $X^{\prime} X$.

As macros completas para os dois exemplos serăo apresentadas no Apendice. 
69.

\section{DISCUSSÃO DE EXEMPLOS NUMÉRICOS}

A partir da metodologia descrita no capitulo anterior, serão construidos, bem como interpretados, os quatro tipos de funçỡes estimáveis fornecidas pelo pacote SAS, em seu PROC GLM, para o caso de dados desbal anceados.

Serão relacionados exemplos, de experimentos fatoriais duplos, com dados desbalanceados e alguns padröes de caselas vazias a fim de observar-se quais hipóteses podem ser mais adequadas a cada situação.

\section{1. 1- GASO: FATORI AL $2 \times 3$ COM UMA GASELA VAZTA}

A Tabela 6 apresenta os dados relativos a um experimento ficticio envol vendo duas variedades de maçã e tres espaçamentos entre plantas. 
Tabel a 6 - Dados em kg de Frutos/Planta

\begin{tabular}{lccc}
\hline & $\begin{array}{c}\text { esp } 1 \\
j=1\end{array}$ & $\begin{array}{c}\text { esp } 2 \\
j=2\end{array}$ & $\begin{array}{c}\text { esp } 3 \\
j=3\end{array}$ \\
\hline$i=1$ & 52,00 & 78,00 & 63,00 \\
Culti var 1 & - & - & 47,00 \\
\hline Total & $52,00(1)$ & $78,00(1)$ & $110,00(2)$ \\
Média & 52,00 & 78,00 & 55,00 \\
\hline $1=2$ & - & 102,00 & 58,00 \\
Cultivar 2 & - & 87,00 & - \\
\hline Total & - & $189,00(2)$ & $58,00(1)$ \\
Média & - & 84,50 & 58,00 \\
\hline Total & $52,00(1)$ & $267,00(3)$ & $168,00(3)$ \\
Média & 52,00 & 89,00 & 56,00 \\
\hline
\end{tabular}

A Tabela 7 apresenta o número de observaçơes por casela.

Tabel a 7 - Número de Observações por Casela Conforme Descrito na Tabela 6

\begin{tabular}{lllll}
\hline & \multicolumn{5}{c}{$J$} \\
\cline { 2 - 5 } & 1 & 2 & 3 & $n_{i}$ \\
\hline 1 & $1 \mu_{11}$ & $1 \mu_{12}$ & $2 \mu_{13}$ & 4 \\
2 & $0 \mu_{2}$ & $2 \mu_{23}$ & 1 & 3 \\
\hline$n \cdot j$ & 1 & 3 & 3 & 7 \\
\hline
\end{tabular}




\subsubsection{Model os Lineares}

Os dados da Tabela 6 podem ser descritos através dos seguintes modelos matemáticos:

- Modelo superparametrizado (Modelo SJ: o modelo dado em (22) assume a caracterização

$$
y_{i j k}=\mu+\alpha_{i}+\beta_{j}+\gamma_{i j}+e_{i j k}
$$

onde:

$y_{i j k}$ é a observação referente a variedade $i$, espaçamento $j$ e k-ésima repetição;

$\mu$ é uma constante inerente aos dados;

$\alpha_{i} \quad$ é o efeito da variedade i Crator $A$;

$\beta_{j} \in$ o efeito do espaçamento $j$ (fator $B$ );

$\gamma_{i j} e 0$ efeito da interação entre variedade $i$ e espaçamento $j(A \times B)$;

$e_{i j k} \Leftrightarrow 0$ erro aleatorio associado a $y_{i j k}$, tal que, $e_{i j k} \cap \mathrm{N}\left(\mathrm{O}, \sigma^{2}\right)$

$i=1,2$

$j=1,2,3$

$k=0, \ldots, n_{i j} \in$ o número de observaçชes por casela

- Modelo de médi as de casel as CModelo MD: o modelo dado em (31) assume a caracterização

$$
y_{i j k}=\mu_{i j}+e_{i j k}
$$

onde:

$y_{i j k} e e_{i j k}$ tém a mesma descrição do modelo anterior;

$\boldsymbol{\mu}_{i j} \quad$ é a média populacional da casela referente à variedade 
72.

i e espaçamento j.

- vetor solução para o Modelo de Médias de Caselas pode ser obtido a partir de:

$$
\begin{gathered}
\mu^{\circ}=\left(w^{\prime} w^{-1} w^{\prime} Y\right. \\
{\left[\begin{array}{l}
\mu_{11}^{0} \\
\mu_{12}^{0} \\
\mu_{13}^{\circ} \\
\mu_{22}^{\circ} \\
\mu_{23}^{\circ}
\end{array}\right]=\left[\begin{array}{l}
\bar{y}_{11} \\
\bar{y}_{12} \\
\bar{y}_{13} \\
\bar{y}_{22} \\
\bar{y}_{23}
\end{array}\right]=\left[\begin{array}{l}
52,00 \\
78,00 \\
55,00 \\
94,50 \\
58,00
\end{array}\right]}
\end{gathered}
$$

$\mu^{\circ}$ será utilizado na obtenção das somas de quadrados de hi póteses.

4.1.2. Obtenção da Forma Geral de Funçōes Estimáveis

Considerando-se o modelo superparametrizado $Y=X \theta+e$, onde:

$$
\mathbf{X}=\left[\begin{array}{ccccccccccc}
\boldsymbol{\mu} & \boldsymbol{\alpha}_{1} & \boldsymbol{\alpha}_{2} & \boldsymbol{\beta}_{1} & \boldsymbol{\beta}_{2} & \boldsymbol{\beta}_{\mathbf{3}} & \boldsymbol{\gamma}_{11} & \boldsymbol{\gamma}_{12} & \boldsymbol{\gamma}_{13} & \boldsymbol{\gamma}_{22} & \boldsymbol{\gamma}_{23} \\
1 & 1 & 0 & 1 & 0 & 0 & 1 & 0 & 0 & 0 & 0 \\
1 & 1 & 0 & 0 & 1 & 0 & 0 & 1 & 0 & 0 & 0 \\
1 & 1 & 0 & 0 & 0 & 1 & 0 & 0 & 1 & 0 & 0 \\
1 & 0 & 0 & 0 & 0 & 1 & 0 & 0 & 1 & 0 & 0 \\
1 & 0 & 1 & 0 & 1 & 0 & 0 & 0 & 0 & 1 & 0 \\
1 & 0 & 1 & 0 & 0 & 1 & 0 & 0 & 0 & 0 & 1
\end{array}\right]
$$


73.

$$
x \cdot x=\left[\begin{array}{lllllllllll}
7 & 4 & 3 & 1 & 3 & 3 & 1 & 1 & 2 & 2 & 1 \\
4 & 4 & 0 & 1 & 1 & 2 & 1 & 1 & 2 & 0 & 0 \\
3 & 0 & 3 & 0 & 2 & 1 & 0 & 0 & 0 & 2 & 1 \\
1 & 1 & 0 & 1 & 0 & 0 & 1 & 0 & 0 & 0 & 0 \\
3 & 1 & 2 & 0 & 3 & 0 & 0 & 1 & 0 & 2 & 0 \\
3 & 2 & 1 & 0 & 0 & 3 & 0 & 0 & 2 & 0 & 1 \\
1 & 1 & 0 & 1 & 0 & 0 & 1 & 0 & 0 & 0 & 0 \\
1 & 1 & 0 & 0 & 1 & 0 & 0 & 1 & 0 & 0 & 0 \\
2 & 2 & 0 & 0 & 0 & 2 & 0 & 0 & 2 & 0 & 0 \\
2 & 0 & 2 & 0 & 2 & 0 & 0 & 0 & 0 & 2 & 0 \\
1 & 0 & 1 & 0 & 0 & 1 & 0 & 0 & 0 & 0 & 1
\end{array}\right]
$$

Para obtenção da matriz inversa generalizada $(\mathrm{X} \cdot \mathrm{X})^{02}$, tomam-se as colunas inearmente independentes sequencialmente da matriz $x$ como sendo a matriz $x_{1}$. Obtem-se $\left(x x_{1}\right)^{-1}$. A matriz $(x \cdot x)^{a 2}$ obtida, colocando as colunas da matriz $\left(\mathrm{X}_{1}^{\prime} \mathrm{X}_{1}\right)^{-1}$ em lugar" das colunas linearmente independentes e intercalando-se com zeros as demais col unas.

$$
\begin{aligned}
x_{1} & =\left[\begin{array}{ccccc}
1 & 1 & 1 & 0 & 0 \\
1 & 1 & 0 & 1 & 1 \\
1 & 1 & 0 & 0 & 0 \\
1 & 1 & 0 & 0 & 0 \\
1 & 0 & 0 & 1 & 0 \\
1 & 0 & 0 & 1 & 0 \\
1 & 0 & 0 & 0 & 0
\end{array}\right] \\
\left(x_{1} x_{1}\right)^{-1} & =\left[\begin{array}{ccccc}
1 & -1 & 0 & -1 & 1 \\
-1 & 3 / 2 & -1 / 2 & 1 & -3 / 2 \\
0 & -1 / 2 & 3 / 2 & 0 & 1 / 2 \\
-1 & 1 & 0 & 3 / 2 & -3 / 2 \\
1 & -3 / 2 & 1 / 2 & -3 / 2 & 3
\end{array}\right]
\end{aligned}
$$


74.

$$
(X \cdot X)^{a 2}=\left[\begin{array}{ccccccccccc}
1 & -1 & 0 & 0 & -1 & 0 & 0 & 1 / 2 & 0 & 0 & 0 \\
-1 & 3 / 2 & 0 & -1 / 2 & 1 & 0 & 0 & -3 / 2 & 0 & 0 & 0 \\
0 & 0 & 0 & 0 & 0 & 0 & 0 & 0 & 0 & 0 & 0 \\
0 & -1 / 2 & 0 & 3 / 2 & 0 & 0 & 0 & 1 / 2 & 0 & 0 & 0 \\
-1 & 1 & 0 & 0 & 3 / 2 & 0 & 0 & -3 / 2 & 0 & 0 & 0 \\
0 & 0 & 0 & 0 & 0 & 0 & 0 & 0 & 0 & 0 & 0 \\
0 & 0 & 0 & 0 & 0 & 0 & 0 & 0 & 0 & 0 & 0 \\
1 & -3 / 2 & 0 & 1 / 2 & -3 / 2 & 0 & 0 & 3 & 0 & 0 & 0 \\
0 & 0 & 0 & 0 & 0 & 0 & 0 & 0 & 0 & 0 & 0 \\
0 & 0 & 0 & 0 & 0 & 0 & 0 & 0 & 0 & 0 & 0 \\
0 & 0 & 0 & 0 & 0 & 0 & 0 & 0 & 0 & 0 & 0
\end{array}\right]
$$

$L=C X \cdot X g^{2}(x \cdot x)$

$$
L=\left[\begin{array}{rrrrrrrrrrrr}
\mu & \alpha_{1} & \alpha_{2} & \beta_{1} & \beta_{2} & \beta_{9} & \gamma_{11} & \gamma_{12} & \gamma_{19} & \gamma_{22} & \gamma_{29} \\
0 & 0 & 1 & 0 & 0 & 1 & 0 & 0 & 0 & 0 & 1 \\
0 & 1 & -1 & 0 & 0 & 0 & 0 & 0 & 1 & 0 & -1 \\
0 & 0 & 0 & 0 & 0 & 0 & 0 & 0 & 0 & 0 & 0 \\
0 & 0 & 0 & 1 & 0 & -1 & 1 & 0 & 1 & 0 & 0 \\
0 & 0 & 0 & 0 & 1 & -1 & 0 & 0 & 0 & 1 & -1 & L_{1} \\
0 & 0 & 0 & 0 & 0 & 0 & 0 & 0 & 0 & 0 & 0 \\
0 & 0 & 0 & 0 & 0 & 0 & 0 & 0 & 0 & 0 & 0 \\
0 & 0 & 0 & 0 & 0 & 0 & 0 & 0 & 0 & 0 & 0 \\
0 & 0 & 0 & 0 & 0 & 0 & 0 & 0 & 0 & 0 & 0 \\
0 & 0 & 0 & 0 & 0 & 0 & 0 & 0 & 0 & 0 & 0
\end{array}\right] L_{5}
$$

Obtendo-se a forma geral das funçర̃es esti máveis através de L $\theta$ que será apresentada na Tabela 8. 
75.

Tabela 8 - Forma Geral de Funçốes Estimáveis para o Modelo Fatorial com Interação Aplicado aos Dados da Tabela 6 .

\begin{tabular}{ll}
\hline Parametros & Coeficiertes \\
\hline$\alpha_{1}$ & $L_{1}$ \\
$\alpha_{2}$ & $L_{2}$ \\
$\beta_{1}$ & $L_{1}-L_{2}$ \\
$\beta_{2}$ & $L_{4}$ \\
$\beta_{9}$ & $L_{5}$ \\
$\gamma_{11}$ & $L_{1}-L_{4}-L_{5}$ \\
$\gamma_{12}$ & $L_{4}$ \\
$\gamma_{19}$ & $L_{8}$ \\
$\gamma_{22}$ & $L_{2}-L_{4}-L_{8}$ \\
$\gamma_{29}$ & $L_{5}-L_{\theta}$ \\
\hline
\end{tabular}

Como cinco simbolos $\mathrm{CL}_{1}, \mathrm{~L}_{2}, \mathrm{~L}_{4}, \mathrm{~L}_{5}, \mathrm{~L}_{8} \mathrm{~g}$ são envolvidos, o posto máximo da hipotese tem cinco graus de 11 berdade.

\subsubsection{Obtençāo das Funçöes Estimáveis Tipo I}

Para a construção das funçశes estimávels tipo I o primeiro passo consiste em fazer a partiçăo da matriz X em $x_{1}, x_{2} e x_{9}$, obedecendo a mesma sequéncia em que os fatores são especificados no modelo, com efeitos principais precedendo interaçбes, de forma a obter o ajuste sequencial. Desta forma: 
76.

$$
x_{1}=\left[\begin{array}{lll}
1 & 1 & 0 \\
1 & 1 & 0 \\
1 & 1 & 0 \\
1 & 1 & 0 \\
1 & 0 & 1 \\
1 & 0 & 1 \\
1 & 0 & 1
\end{array}\right]
$$

que formada pelas colunas da matriz $X$ correspondentes a $\mu$ - ao fator A (variedades).

$$
x_{2}=\left[\begin{array}{lll}
1 & 0 & 0 \\
0 & 1 & 0 \\
0 & 0 & 1 \\
0 & 0 & 1 \\
0 & 1 & 0 \\
0 & 1 & 0 \\
0 & 0 & 1
\end{array}\right]
$$

que $E$ formada pelas col unas de $X$ correspondentes ao fator $B$ (espaçamentos).

$$
X_{3}=\left[\begin{array}{lllll}
1 & 0 & 0 & 0 & 0 \\
0 & 1 & 0 & 0 & 0 \\
0 & 0 & 1 & 0 & 0 \\
0 & 0 & 1 & 0 & 0 \\
0 & 0 & 0 & 1 & 0 \\
0 & 0 & 0 & 1 & 0 \\
0 & 0 & 0 & 0 & 1
\end{array}\right]
$$

- formada pela colunas de $X$ correspondentes a interaçăo entre variedades e espaçamentos.

Para obtenção das funçбes estimávels tipo I para o fator A (variedades) se construirá a seguinte matriz:

$$
\begin{gathered}
G_{1}=C x_{1} x_{1}\left|x_{1} x_{2}\right| x_{1} x_{9}{ }^{2}= \\
=\left[\begin{array}{lll|lll|lllll}
7 & 4 & 3 & 1 & 3 & 3 & 1 & 1 & 2 & 2 & 1 \\
4 & 4 & 0 & 1 & 1 & 2 & 1 & 1 & 2 & 0 & 0 \\
3 & 0 & 3 & 0 & 2 & 1 & 0 & 0 & 0 & 2 & 1
\end{array}\right]
\end{gathered}
$$


então $\quad G_{1}^{*}=\left(x_{1} \cdot x_{1}\right)^{-} G_{1}=$

$$
=\left[\begin{array}{ccc|ccc|ccccc}
\mu & \alpha_{1} & \alpha_{2} & \beta_{1} & \beta_{2} & \beta_{3} & \gamma_{11} & \gamma_{12} & \gamma_{13} & \gamma_{22} & \gamma_{29} \\
0 & 0 & 1 & 0 & 2 / 3 & 1 / 3 & 0 & 0 & 0 & 2 / 3 & -1 / 3 \\
0 & 0 & 0 & 0 & 0 & 0 & 0 & 0 & 0 & 0 & 0
\end{array}\right] \begin{gathered}
L_{1} \\
L_{2}
\end{gathered}
$$

Em termos da notação RC J, a soma de quadrados tipo I, correspondente a funçăo estimável tipo I, para o fator A é, como já visto, dada por $R(\alpha \mid \mu)$. Em função disto a primeira linha de $G_{1}^{*}$ que corresponde ao parametro $\mu$ será tomada como zero.

$$
G_{1}^{*} \theta \text { fornece as funçores estimáveis tipo I para }
$$
A apresentadas na Tabela $\theta$.

Tabel a 9 - Funçб̋es Estimáveis Tipo I para A.

\begin{tabular}{cc}
\hline Parametros & Coeficientes \\
\hline$\mu_{1}$ & 0 \\
$\alpha_{2}$ & $\mathrm{~L}_{2}$ \\
$\beta_{1}$ & $-\mathrm{L}_{2}$ \\
$\beta_{2}$ & $1 / 4 \mathrm{~L}_{2}$ \\
$\beta_{13}$ & $-5 / 12 \mathrm{~L}_{2}$ \\
$\gamma_{11}$ & $1 / 8 \mathrm{~L}_{2}$ \\
$\gamma_{12}$ & $1 / 4 \mathrm{~L}_{2}$ \\
$\gamma_{19}$ & $1 / 4 \mathrm{~L}_{2}$ \\
$\gamma_{22}$ & $1 / 2 \mathrm{~L}_{2}$ \\
$\gamma_{29}$ & $-2 / 3 \mathrm{~L}_{2}$ \\
\hline
\end{tabular}

Atribuindo-se valores arbitrarios a $L_{2}$ se 
78.

obtera a hipotese testada atraves da soma de quadrados tipo I que o PROC GLM do SAS fornece.

Fazendo-se $\mathrm{L}_{2} 1$ gual a 1 , por exemplo, obtém-se:

$H_{0}^{(1)}: \alpha_{1}-\alpha_{2}+1 / 4 \beta_{1}-5 / 12 \beta_{2}+1 / 6 \beta_{9}+1 / 4 \gamma_{11}+1 / 4 \gamma_{12}+$

$$
+1 / 2 \gamma_{19}-2 / 3 \gamma_{22}-1 / 3 \gamma_{29}=0
$$

e, para qualquer outro valor de $L_{2}$ serăo obtidas hipóteses equi valentes.

A hipótese tipo I para A envolve além dos parametros $\alpha$, os parametros $\beta$ e pois a soma de quadrados tipo I equivale a $R(\propto \mid \mu)$, năo sendo ajustada para o fator $B$ e interação.

E uma hipotese sobre médias ponderadas de

11 nhas.

Esta hipótese tem a-1 = 1 grau de liberdade e por 1sto um único símbolo L está associado a ela.

A hipotese $\mathrm{Ho}^{(4)}$ pode ser escrita em termos do Modelo de Médias de Casel as ficando:

$$
H_{0}^{(1)}: \frac{1}{4} \mu_{11}+\frac{1}{4} \mu_{12}+\frac{1}{2} \mu_{19}-\frac{2}{3} \mu_{22}-\frac{1}{3} \mu_{29}=0
$$

Nota-se que a hipótese escrita em termos do Modelo $M$ e mais facil de interpretar.

Observa-se que estão sendo comparadas as medias da linha um com as medias da linha dois. Porem, envol vendo coeficientes um pouco confusos, por tratar-se de uma hipótese sobre médias ponderadas em presença de frequências diferentes cdiferentes repetiçôs e caselas vazias?. 
79.

A Soma de Quadrados tipo I para A pode ser obtida da seguinte forma

$$
\left.R(\alpha \mid \mu)=S Q H H^{\langle 1\rangle}=\left[B_{\langle 1\rangle^{\prime}}^{\prime}\right]^{0}\right]^{\prime}\left[\mathrm{B}_{\langle 1\rangle^{\prime}}\left(W^{\prime} W^{-1} \mathrm{~B}_{\langle 1\rangle}\right]^{-1}\left[\mathrm{~B}_{\langle 1\rangle^{\prime}} \mu^{0}\right]\right.
$$

onde $B_{\text {(1) }}$ a matriz dos coeficiente dos parametros na hipotese, dada por

$$
B_{(1)}^{\prime}=\left[\begin{array}{lllll}
\frac{1}{4} & \frac{1}{4} & \frac{1}{2} & -\frac{2}{3} & -\frac{1}{3}
\end{array}\right]
$$

W a matriz de delineamento no Modelo $M$

$\mu^{\circ} \leqslant$ o vetor soluçá de $\mu^{0}=\left(W^{\prime} W^{-1} W^{\prime} Y\right.$, estimativas das médias de caselas.

$$
R(\alpha \mid \mu)=S O H_{0}^{(1)}=855,048
$$

As funçశes estimareis tipo I para o fator $B$

(espaçamentol são obtidas atravess de

$$
\begin{aligned}
& G_{2}=c 0\left|X_{2}^{\prime} M_{1} X_{2}\right| X_{2} \cdot M_{1} X_{9}{ }^{2} \\
& M_{1}=I-X_{1}\left(X_{1}^{\prime} X_{1}\right)^{-} X_{1}^{\prime} \\
& M_{1}=\left[\begin{array}{ccccccc}
3 / 4 & -1 / 4 & -1 / 4 & -1 / 4 & 0 & 0 & 0 \\
-1 / 4 & 3 / 4 & -1 / 4 & -1 / 4 & 0 & 0 & 0 \\
-1 / 4 & -1 / 4 & 3 / 4 & -1 / 4 & 0 & 0 & 0 \\
-1 / 4 & -1 / 4 & -1 / 4 & 3 / 4 & 0 & 0 & 0 \\
0 & 0 & 0 & 0 & 2 / 3 & -1 / 3 & -1 / 3 \\
0 & 0 & 0 & 0 & -1 / 3 & 2 / 3 & -1 / 3 \\
0 & 0 & 0 & 0 & -1 / 3 & -1 / 3 & 2 / 3
\end{array}\right]
\end{aligned}
$$

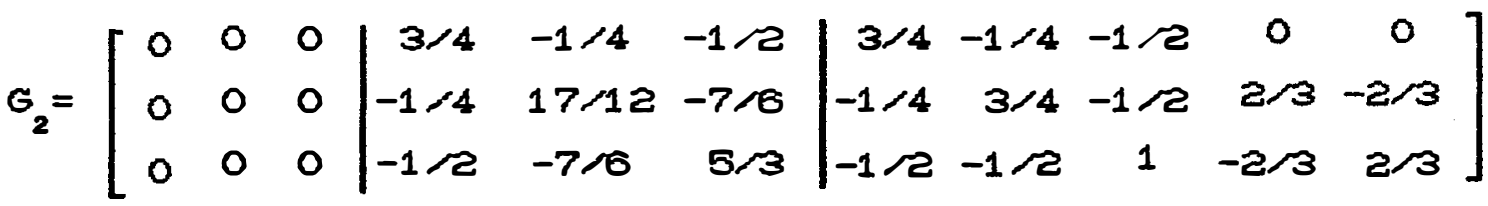


80.

$$
\begin{aligned}
& G_{2}^{*}=\left(X_{2} M_{1} X_{2}\right)^{-} G_{2}= \\
& =\left[\begin{array}{ccc|cccccccc}
\mu & \alpha_{1} & \alpha_{2} & \beta_{1} & \beta_{2} & \beta_{9} & \gamma_{11} & \gamma_{12} & \gamma_{19} & \gamma_{22} & \gamma_{29} \\
0 & 0 & 0 & 1 & 0 & -1 & 1 & -1 / \sigma & -5 / 6 & 1 / \sigma & -1 / \sigma \\
0 & 0 & 0 & 0 & 1 & -1 & 0 & 1 / 2 & -1 / 2 & 1 / 2 & -1 / 2 \\
0 & 0 & 0 & 0 & 0 & 0 & 0 & 0 & 0 & 0
\end{array}\right] L_{4}^{L_{4}} \\
& G_{2}^{*} \theta \text { fornece as funçชes estimáveis tipo I para }
\end{aligned}
$$

Tabel a 10 - Funçỡes Estimáveis Tipo I para B.

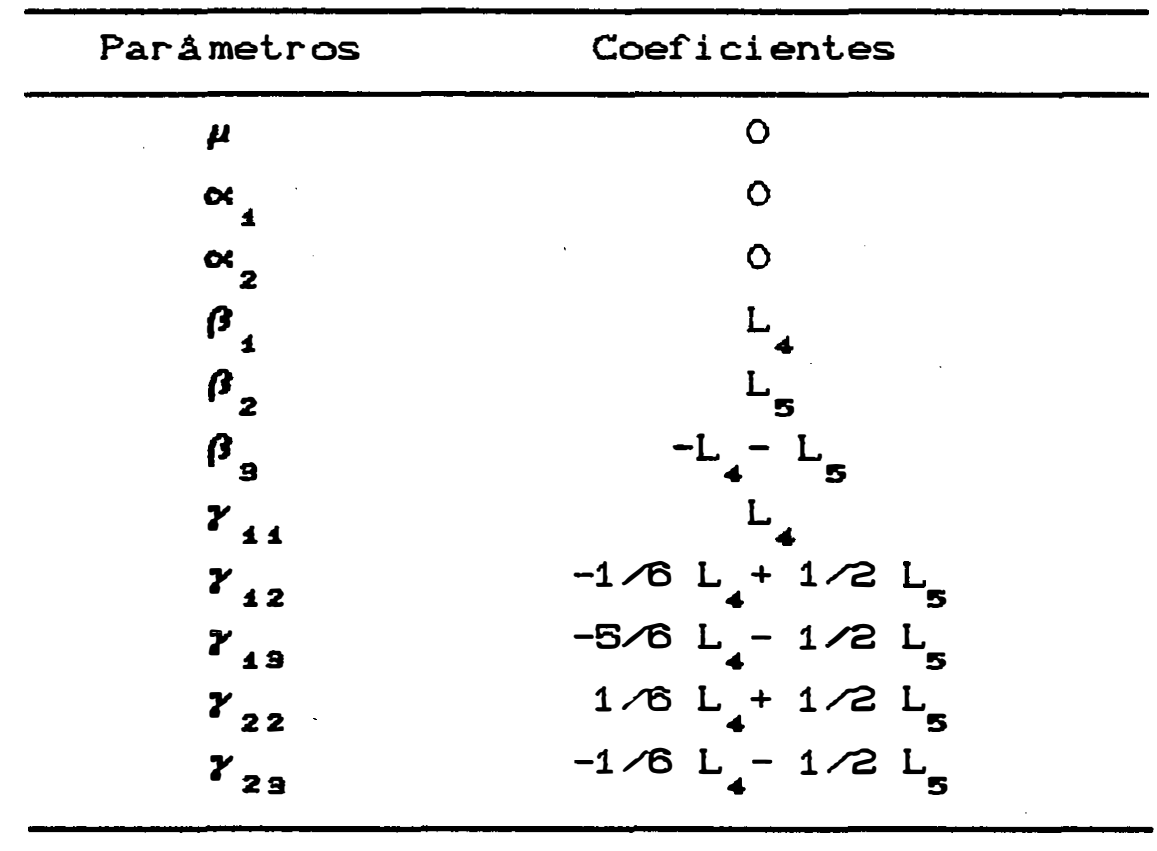

Atribuindo-se valores arbitrários a $L_{4} \in L_{5}$. se obterá uma hipotese testada atraves da soma de quadrados tipo I que o sas fornece. Fazendo-se, por exemplo, $L_{4}=1$ e $L_{5}=0$, depois $L_{4}=0$ e $L_{5}=1$, obtém-se:

$$
H_{0}^{(5)}: \begin{cases}\beta_{1} & -\beta_{9}+\gamma_{11}-\frac{1}{6} \gamma_{12}-\frac{5}{6} \gamma_{19}+\frac{1}{\delta} \gamma_{22}-\frac{1}{6} \gamma_{29}=0 \\ & \beta_{2}-\beta_{9}+\frac{1}{2} \gamma_{12}-\frac{1}{2} \gamma_{19}+\frac{1}{2} \gamma_{22}-\frac{1}{2} \gamma_{29}=0\end{cases}
$$

A hipótese tipo I para o fator $B$ năo envolve os parametros $\alpha$, pois para as somas de quadrados tipo I o 
81.

ajuste é sequencial. Equivalendo assim a $R(\beta \mid \mu, \alpha)$, quando 0 modelo é especificado na ordem $A, B, A * B$.

E uma hipótese sobre médias ponderadas de col unas ajustadas para linhas.

Esta hipótese tem b- $b=2$ graus de liberdade e por isso dois símbolos L associados a ela.

A hipótese $H_{0}^{(5)}$ também pode ser escrita em termos do Modelo de Médias de Caselas, ficando:

$$
H_{0}^{(5)}:\left\{\begin{array}{r}
\mu_{11}-\frac{1}{\sigma} \mu_{12}-\frac{5}{\sigma} \mu_{13}+\frac{1}{\sigma} \mu_{22}-\frac{1}{\sigma} \mu_{23}=0 \\
\frac{1}{2} \mu_{12}-\frac{1}{2} \mu_{13}+\frac{1}{2} \mu_{22}-\frac{1}{2} \mu_{23}=0
\end{array}\right.
$$

A primeira linha da hipótese é um contraste entre coluna um, contra dois e tres, dentro da linha um e coluna dois contra tres dentro da linha dois. E de interpretação confusa.

A segunda linha $e$ mais simples e envolve comparação entre colunas dois e trés em ambas as 1 inhas.

A Soma de Quadrados tipo I para B pode ser obtida de:

$$
R(\beta \mid \mu, \alpha)=S Q H 0^{(5)}=\left[B_{(5)^{\prime}}^{\prime}\right]^{\circ}\left[B_{(5)^{\prime}}\left(W^{\prime} W\right)^{-1} B_{(5)}\right]^{-1}\left[B_{(5)^{\prime}} \mu^{0}\right]
$$

onde:

$$
\begin{aligned}
B_{(5)}^{\prime}=\left[\begin{array}{ccccc}
1 & -1 / \sigma & -5 / 6 & 1 / 6 & -1 / \sigma \\
0 & 1 / 2 & -1 / 2 & 1 / 2 & -1 / 2
\end{array}\right] \\
R(\beta \mid \mu, \alpha)=S Q H^{(5)}=1265,417 \\
\text { As funçชes estimaveis tipo I para a interação }
\end{aligned}
$$


82.

entre variedades e espaçamentos são obtidas através de:

$$
\begin{aligned}
& G_{3}=C O P|O| X_{3} M_{2} X_{3}^{3} \\
& M_{2}=M_{1}-M_{1} X_{2} C X_{2}^{\prime} M_{1} X_{2} 3^{-} X_{2}^{\prime} M_{1} \\
& M_{2}=\left[\begin{array}{cccccccc}
0 & 0 & 0 & 0 & 0 & 0 & 0 \\
0 & 1 / 3 & -1 / 6 & -1 / 6 & -1 / 6 & -1 / 6 & 1 / 3 \\
0 & -1 / 6 & 7 / 12 & -5 / 12 & 1 / 12 & 1 / 12 & -1 / 6 \\
0 & -1 / 6 & -5 / 12 & 7 / 12 & 1 / 12 & 1 / 12 & -1 / 6 \\
0 & -1 / 6 & 1 / 12 & 1 / 12 & 7 / 12 & -5 / 12 & -1 / 6 \\
0 & -1 / 6 & 1 / 12 & 1 / 12 & 1 / 12 & -1 / 6 & 1 / 12 \\
0 & 1 / 3 & -1 / 6 & -1 / 6 & -1 / 6 & 1 / 3 & -1 / 6
\end{array}\right] \\
& G_{9}=\left[\begin{array}{lll|lll|lllll}
0 & 0 & 0 & 0 & 0 & 0 & 0 & 0 & 0 & 0 & 0 \\
0 & 0 & 0 & 0 & 0 & 0 & 0 & 1 / 3 & -1 / 3 & -1 / 3 & 1 / 3 \\
0 & 0 & 0 & 0 & 0 & 0 & 0 & -1 / 3 & 1 / 3 & 1 / 3 & -1 / 3 \\
0 & 0 & 0 & 0 & 0 & 0 & 0 & -1 / 3 & 1 / 3 & 1 / 12 & -1 / 12 \\
0 & 0 & 0 & 0 & 0 & 0 & 0 & 1 / 3 & -1 / 3 & 1 / 6 & -1 / 6
\end{array}\right] \\
& G_{9}^{*}=\left(X_{3} M_{2} X_{9}\right)^{-} G_{9} \\
& G_{3}^{*}=\left[\begin{array}{lll|lll|l|lcccc}
0 & 0 & 0 & 0 & 0 & 0 & 0 & 0 & 0 & 0 & 0 \\
0 & 0 & 0 & 0 & 0 & 0 & 0 & 1 & -1 & -1 & 1 \\
0 & 0 & 0 & 0 & 0 & 0 & 0 & 0 & 0 & 0 & 0 \\
0 & 0 & 0 & 0 & 0 & 0 & 0 & 0 & 0 & 0 & 0 \\
0 & 0 & 0 & 0 & 0 & 0 & 0 & 0 & 0 & 0 & 0
\end{array}\right] L_{B} \\
& G_{9}^{*} \theta \text { fornece as funçơes estimáveis tipo I para } \\
& \text { interação } A \times B \text { apresentadas na Tabela } 11 .
\end{aligned}
$$


83.

Tabela 11 - Funçđ̃es Estimáveis Típo I para AxB.

\begin{tabular}{cc}
\hline Parametros & Coeficientes \\
\hline$\mu_{1}$ & 0 \\
$\alpha_{2}$ & 0 \\
$\beta_{1}$ & 0 \\
$\beta_{2}$ & 0 \\
$\beta_{9}$ & 0 \\
$\gamma_{11}$ & 0 \\
$\gamma_{12}$ & 0 \\
$\gamma_{19}$ & $\mathrm{~L}_{\theta}$ \\
$\gamma_{22}$ & $-\mathrm{L}_{\theta}$ \\
$\gamma_{29}$ & $-\mathrm{L}_{\theta}$ \\
\end{tabular}

Como um simbolo $\mathrm{L}\left(\mathrm{L}_{\mathrm{\theta}} \mathrm{O}\right.$ aparece nas funçб̃es estimáveis para AxB, a hipótese associada a esta tem apenas um grau de 1 iberdade.

Alén disso, segundo IEMMA (1993), quando ocorrem caselas vazias, o número de graus de liberdade da interação é dado por

$$
s-(a+b-1)=5-4=1
$$

onde $s$ é número de caselas completas, a e o número de niveis do fator $A$ e $b$ e número de niveis do fator $B$.

Hipóteses tipo I para a interação AxB podem ser obtidas atribuindo-se valores arbitrários a $L_{\theta}$. Assim, se $L_{0}$ igual a 1

$$
\mathrm{Ho}^{(7)}: \gamma_{12}-\gamma_{19}-\gamma_{22}+\gamma_{29}=0
$$

Observa-se que a hipótese sobre a interação é 
livre dos outros parametros. Isto reitera o fato de que o modelo com interação é adequado para testar a interação. Porém, quando ocorrem caselas vazias nem todas as interaçơes possiveis são testadas. Neste caso, a hipótese não envolve $\boldsymbol{\gamma}_{11}$ e $\boldsymbol{\gamma}_{21}$, e nada se pode afirmar sobre eles.

Em termos do Modelo $M$ a hipótese fica:

$$
\mathrm{Ho}^{(7)}: \mu_{12}-\mu_{19}-\mu_{22}+\mu_{29}=0
$$

Para obter-se a Soma de Quadrados tipo I para

a interação AxB, calcula-se

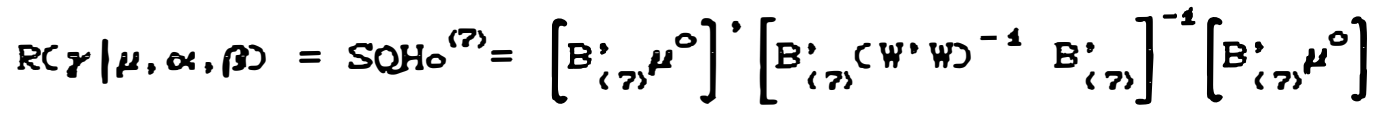

onde

$$
\begin{gathered}
B_{(z)}^{\prime}=\left[\begin{array}{lllll}
0 & 1 & -1 & -1 & 1
\end{array}\right] \\
R C \gamma \mid \mu, \alpha, \beta)=S O H_{0}^{(\beta)}=60.750
\end{gathered}
$$

\subsection{4, Obtenção das Funçōes Estimáveis Tipo II}

Para obtençăo das funçơes estimáveis tipo II para o fator A deverao ser feitas as seguintes consi der açช̃es:

- o efeito do fator A está contido no efeito da interação AxB;

- o efeito do fator A não está contido no efeito do fator B. Assim, as funçชes estimáveis tipo II para A são construidas da seguinte forma:

- as colunas de $L$ associadas ao fator $B$ serão preenchidas por zeros; 
85.

- a submatriz associada com o fator A será

$$
\left(\mathrm{X}_{1} \mathrm{MX}_{1}\right)^{-}\left(\mathrm{X}_{1} \cdot \mathrm{M} \mathrm{X}_{1}\right)^{\prime}
$$

- a submatriz associada com o fator AxB sera

$$
\left(X_{1} \operatorname{Mx}_{1}\right)^{-}\left(x_{1} \mathrm{Mx}_{2}\right)
$$

onde:

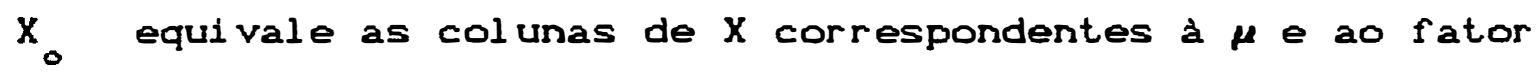
B Cefeitos que não contém $A$.

$$
x_{0}=\left[\begin{array}{llll}
1 & 1 & 0 & 0 \\
1 & 0 & 1 & 0 \\
1 & 0 & 0 & 1 \\
1 & 0 & 0 & 1 \\
1 & 0 & 1 & 0 \\
1 & 0 & 1 & 0 \\
1 & 0 & 0 & 1
\end{array}\right]
$$

$X_{1}$ equivale as colunas de $X$ associadas ao fator $A$

$$
x_{1}=\left[\begin{array}{ll}
1 & 0 \\
1 & 0 \\
1 & 0 \\
1 & 0 \\
0 & 1 \\
0 & 1 \\
0 & 1
\end{array}\right]
$$

$X_{2}$ equivale as colunas de $X$ associadas a interaçăo $A \times B$ que contém $A$

$$
\begin{aligned}
& X_{2}=\left[\begin{array}{lllll}
1 & 0 & 0 & 0 & 0 \\
0 & 1 & 0 & 0 & 0 \\
0 & 0 & 1 & 0 & 0 \\
0 & 0 & 1 & 0 & 0 \\
0 & 0 & 0 & 1 & 0 \\
0 & 0 & 0 & 1 & 0 \\
0 & 0 & 0 & 0 & 1
\end{array}\right] \\
& M=I-X_{0}\left(X_{0} X_{0}\right)-X_{0}^{\prime}
\end{aligned}
$$


86.

$$
\begin{aligned}
& M=\left[\begin{array}{ccccccc}
0 & 0 & 0 & 0 & 0 & 0 & 0 \\
0 & 2 / 3 & 0 & 0 & -1 / 3 & -1 / 3 & 0 \\
0 & 0 & 2 / 3 & -1 / 3 & 0 & 0 & -1 / 3 \\
0 & 0 & -1 / 3 & 2 / 3 & 0 & 0 & -1 / 3 \\
0 & -1 / 3 & 0 & 0 & 2 / 3 & -1 / 3 & 0 \\
0 & -1 / 3 & 0 & 0 & -1 / 3 & 2 / 3 & 0 \\
0 & 0 & -1 / 3 & -1 / 3 & 0 & 0 & 2 / 3
\end{array}\right] \\
& \beta \alpha_{1} \alpha_{2} \beta_{1} \beta_{2} \beta_{3} \gamma_{11} \gamma_{12} \gamma_{19} \gamma_{22} \gamma_{23} \\
& L=\left[\begin{array}{ccc|ccc|ccccc}
0 & 0 & 0 & 0 & 0 & 0 & 0 & 0 & 0 & 0 & 0 \\
0 & 1 & -1 & 0 & 0 & 0 & 0 & 1 / 2 & 1 / 2 & -1 / 2 & -1 / 2
\end{array} L_{2}\right.
\end{aligned}
$$

Através de Le obtém-se as funçớes estimáveis tipo II para o fator A (variedades), apresentadas na Tabela 12 .

Tabel a 12 - Funçỡes Estimáveis Tipo II para A.

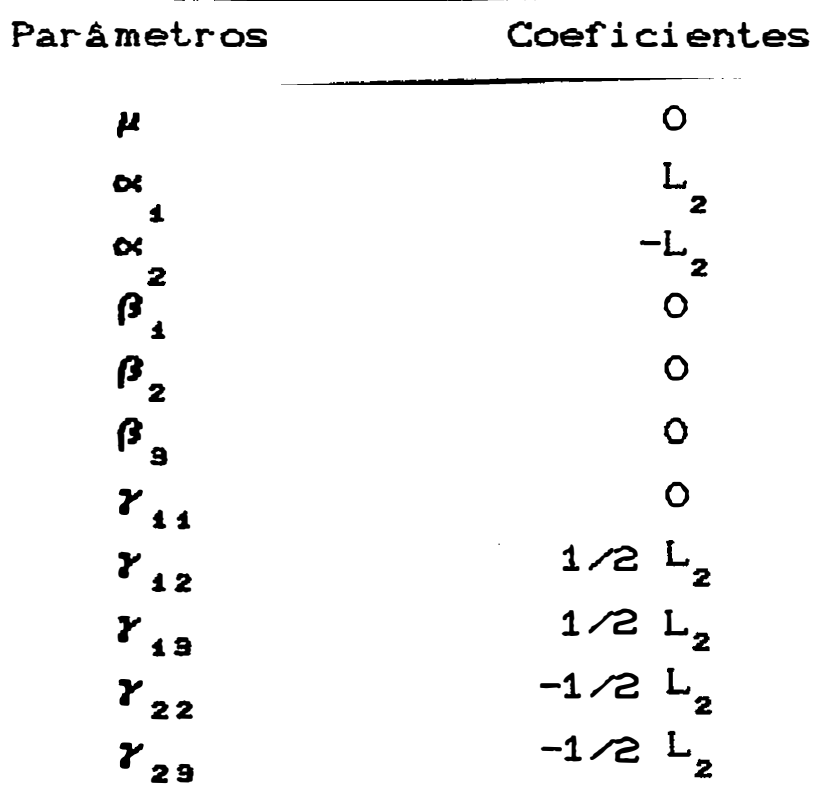

Atribuindo-se valores arbitrários a $L_{2}$, obtem-se as hipóteses tipo II para A. Fazendo-se $L_{2}$ igual 
87.

a 1

$$
H_{0}^{(2)}: \alpha_{1}-\alpha_{2}+\frac{1}{2} \gamma_{12}+\frac{1}{2} \gamma_{13}-\frac{1}{2} \gamma_{22}-\frac{1}{2} \gamma_{29}=0
$$

A hipotese tipo II para A não envolve os parametros $\beta$, pois a soma de quadrados tipo II para A se equivale a $R C \alpha \mid \mu, \beta)$.

Trata-se de uma hipótese sobre médias ponderadas de linhas ajustadas para colunas.

A hipótese $\mathrm{Ho}^{(2)}$ em termos do modelo de médias de caselas fica

$$
\begin{aligned}
& H_{0}^{(2)}: \frac{1}{2} \mu_{12}+\frac{1}{2} \mu_{19}-\frac{1}{2} \mu_{22}-\frac{1}{2} \mu_{29}=0 \\
& H_{0}^{(2)} \text { compara linhas um } e \text { dois considerando }
\end{aligned}
$$
somente colunas dois e trés.

Para obtenção da Soma de quadrados tipo II para A, calcula-se

$$
R C \alpha \mid \mu, \beta)=S O H 0^{(2)}=\left[B_{(2)^{\prime}}^{\prime} \mu^{0}\right]^{\prime}\left[B_{(2)}^{\prime}\left(W^{\prime}, W\right)^{-1} B_{(2)}\right]^{-1}\left[B_{(2)^{\prime}}^{\prime} \mu^{0}\right]
$$

onde $\quad B_{(2)}^{\prime}=\left[\begin{array}{lllll}0 & \frac{1}{2} & \frac{1}{2} & -\frac{1}{2} & -\frac{1}{2}\end{array}\right]$

$$
\mathrm{R}(\alpha \mid \mu, \beta)=\mathrm{SOH}_{0}^{(2)}=126,750
$$

Da mesma forma, para obtenção das funçб̃es estimáveis tipo II para B, considera-se que o efeito do fator $B$ está contido no efeito da interação AxB e não está contido no efeito do fator $A$. 
88.

Portanto

$$
\begin{aligned}
& X_{0}=\left[\begin{array}{lll}
1 & 1 & 0 \\
1 & 1 & 0 \\
1 & 1 & 0 \\
1 & 1 & 0 \\
1 & 0 & 1 \\
1 & 0 & 1 \\
1 & 0 & 1
\end{array}\right] ; \quad X_{1}=\left[\begin{array}{lll}
1 & 0 & 0 \\
0 & 1 & 0 \\
0 & 0 & 1 \\
0 & 0 & 1 \\
0 & 1 & 0 \\
0 & 1 & 0 \\
0 & 0 & 1
\end{array}\right] ; X_{2}=\left[\begin{array}{lllll}
1 & 0 & 0 & 0 & 0 \\
0 & 1 & 0 & 0 & 0 \\
0 & 0 & 1 & 0 & 0 \\
0 & 0 & 1 & 0 & 0 \\
0 & 0 & 0 & 1 & 0 \\
0 & 0 & 0 & 1 & 0 \\
0 & 0 & 0 & 0 & 1
\end{array}\right]
\end{aligned}
$$

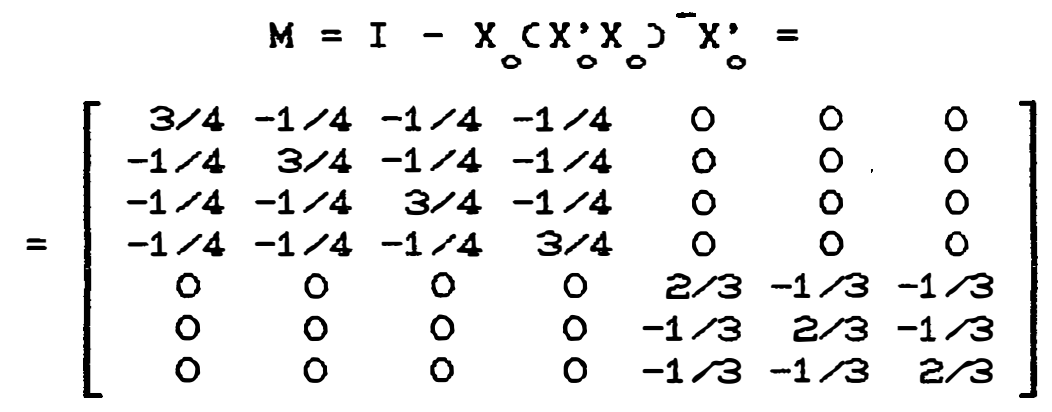

$$
L=\left[\begin{array}{lll|llll|lcccc}
0 & 0 & 0 & 1 & 0 & -1 & 1 & -1 / \theta & -5 / \sigma & 1 / 6 & -1 / \sigma \\
0 & 0 & 0 & 0 & 1 & -1 & 0 & 1 / 2 & -1 / 2 & 1 / 2 & -1 / 2 \\
0 & 0 & 0 & 0 & 0 & 0 & 0 & 0 & 0 & 0 & 0
\end{array}\right] L_{5}^{L_{4}}
$$

Através de Le obtém-se as funçశ̋ estimáveis tipo II para o fator B (espaçamentos), apresentadas na Tabela 13. 
89.

Tabela 13 - Funçס̃es Estimáveis Tipo II para B.

\begin{tabular}{cc}
\hline Parametros & Coeficientes \\
\hline$\mu_{1}$ & 0 \\
$\alpha_{1}$ & 0 \\
$\alpha_{2}$ & 0 \\
$\beta_{1}$ & $L_{4}$ \\
$\beta_{2}$ & $L_{5}$ \\
$\beta_{9}$ & $-L_{4}-L_{5}$ \\
$\gamma_{11}$ & $L_{4}$ \\
$\gamma_{12}$ & $-1 / 6 L_{4}+1 / 2 L_{5}$ \\
$\gamma_{19}$ & $-5 / 6 L_{4}-1 / 2 L_{5}$ \\
$\gamma_{22}$ & $1 / 6 L_{4}+1 / 2 L_{5}$ \\
$\gamma_{29}$ & $-1 / 6 L_{4}-1 / 2 L_{5}$ \\
\hline
\end{tabular}

Atribuindo-se valores a $L_{4}$ e $L_{5}$ obtém-se as hipoteses tipo II para o fator B.

Fazendo-se $L_{4}=1$ e $L_{5}=0$, depois $L_{4}=0$ e $L_{5}=1$, obtém-se

$$
H 0^{(5)}:\left\{\begin{array}{l}
\beta_{1}-\beta_{9}+\gamma_{11}-\frac{1}{6} \gamma_{12}-\frac{5}{\delta} \gamma_{19}+\frac{1}{\delta} \gamma_{22}-\frac{1}{6} \gamma_{29}=0 \\
\beta_{2}-\beta_{9}+\frac{1}{2} \gamma_{12}-\frac{1}{2} \gamma_{19}+\frac{1}{2} \gamma_{22}-\frac{1}{2} \gamma_{29}=0
\end{array}\right.
$$

Observa-se que esta hipótese e a mesma hipotese tipo I para B.

Ambas são testadas por $R(\beta \mid \mu, \alpha)$.

As funçớes estimáveis tipos I e II para o fator B săo iguais devido ao ajuste sequencial das $f$ unçত̃es estimaveis tipo I.

Para a obtenção das funçன̆es estimáveis tipo 
90.

I para a interação AxB, basta tomar a forma geral de funçỡes estimáveis, apresentada na Tabela 8 , igualar $L_{1}, L_{2}$. $L_{4} e L_{5}$ a zero.

$$
\text { Obtendo-se o seguinte: }
$$

Tabel a 14 - Funçỡes Estimáveis Tipo II para AxB.

\begin{tabular}{cc}
\hline Parametros & Coeficientes \\
\hline$\alpha_{1}$ & 0 \\
$\alpha_{2}$ & 0 \\
$\beta_{1}$ & 0 \\
$\beta_{2}$ & 0 \\
$\beta_{3}$ & 0 \\
$\gamma_{11}$ & 0 \\
$\gamma_{12}$ & 0 \\
$\gamma_{19}$ & $\mathrm{~L}_{8}$ \\
$\gamma_{22}$ & $-\mathrm{L}_{\mathrm{B}}$ \\
$\gamma_{29}$ & $-\mathrm{L}_{\theta}$ \\
\hline
\end{tabular}

Observa-se que as funçб̋es estimáveis tipo II para a interação $A \times B$ são iguais as tipo I. Isto ocorre também para as tipos III e IV. Todos os quatro tipos de somas de quadrados para a interação se equivalem e são iguais a $R(\gamma \mid \mu, \alpha, \beta)$. Isto $\Leftrightarrow$, a hipótese sobre a interação $\Leftrightarrow$ sempre ajustada para os demais fatores.

4. 1.5. Obtenção das fumçōes estimáveis tipo III

Para obtenção das funçơes estimáveis tipo 
91 .

III, trabalhar-se-a também com a forma geral de funçôes estimáveis, já apresentada na Tabela 8.

As funçơes estimáveis tipo III para o fator A (variedades) são construídas, segundo o Manual do SAS, Capitulo 9 (1990), de acordo com os seguintes passos:

- para cada efeito no modelo, exceto o fator $A$ e AxB, que contém A, igualar os coeficientes na forma geral de funçơes estimáveis a zero. Assim, $L_{1}, L_{4} e L_{5}$ são considerados iguais a zero;

- igualar os coeficientes fora do bloco do fator A a uma função linear dos coeficientes do bloco do fator A, a fim de fazer a hipótese de A ortogonal a hipótese associada à AxB.

Fazendo-se $\mathrm{L}_{\mathbf{B}}=\mathrm{K} \mathrm{L}_{2} e$ encontrando-se 0 valor de $\mathrm{K}$ que torne a hipótese de $\mathrm{A}$ ortogonal a hipótese de AxB. Neste caso, $k=1 / 2$, pois o coeficiente $L_{2}$ de $\alpha_{1}$ será distribuido entre dois niveis de $A \times B$ e o coeficiente $-L_{2}$ de $\alpha_{2}$, também.

As funçơes estimáveis tipo III para o fator $A$ resultantes deste processo são apresentadas na Tabela 15. 
92.

Tabela 15 - Funçбes Estimáveis Tipo III para A.

\begin{tabular}{cc}
\hline Parametros & Coeficientes \\
\hline$\mu_{1}$ & 0 \\
$\alpha_{2}$ & $-L_{2}$ \\
$\beta_{1}$ & 0 \\
$\beta_{2}$ & 0 \\
$\beta_{3}$ & 0 \\
$\gamma_{11}$ & 0 \\
$\gamma_{12}$ & $L_{8}=1 / 2 L_{2}$ \\
$\gamma_{13}$ & $L_{2}-L_{8}=1 / 2 L_{2}$ \\
$\gamma_{22}$ & $-L_{9}=-1 / 2 L_{2}$ \\
$\gamma_{23}$ & $-L_{2}+L_{\theta}=-1 / 2 L_{2}$ \\
\hline
\end{tabular}

Somando-se os coeficientes de $\boldsymbol{\gamma}_{11}$, $\boldsymbol{\gamma}_{12}$ e $\boldsymbol{\gamma}_{13}$ ' obtém-se $L_{2}$ que é 0 coeficiente de $\alpha_{1}$. Somando-se os coeficientes de $\boldsymbol{\gamma}_{22}$ e $\boldsymbol{\gamma}_{23}$, obtém-se $-L_{2}$ que é o coeficiente de $\alpha_{2}$. Da mesma forma, a soma dos coeficientes de $\gamma_{12} \theta$ $\boldsymbol{\gamma}_{22}$ deve ser zero (coeficiente de $\left.\beta_{2}\right), \boldsymbol{\gamma}_{13}$ mais $\boldsymbol{\gamma}_{23}$ deve ser igual a zero e $\boldsymbol{\gamma}_{11}$ também.

Atribuindo-se valores a $L_{2}$ serão obtidas hipóteses tipo III para o fator A.

$$
\begin{aligned}
& \text { Assim, fazendo-se } L_{2} \text { igual a } 1 \\
& \mathrm{Ho}^{(3)}: \alpha_{1}-\alpha_{2}+\frac{1}{2} \gamma_{12}+\frac{1}{2} \gamma_{13}-\frac{1}{2} \gamma_{22}-\frac{1}{2} \gamma_{23}=0
\end{aligned}
$$

que é uma hipótese sobre médias não ponderadas de linhas.

As funçত̋es estimáveis tipo III são obtidas com base no Modelo $\boldsymbol{\Sigma}$ ou Modelo com Restriça nos Parametros. Por isso, se equivalem em termos da notação RC 2 , a $R C \dot{\alpha} \mid \dot{\mu}, \dot{\beta}, \dot{\gamma})$ 
83.

A hipótese tipo III para A em termos dos parámetros do modelo restrito é dada por

$$
H_{0}^{(9)}: \quad \dot{\alpha}_{i}=0 \quad \forall i
$$

e escrevendo-se esta hipótese em termos dos parámetros do

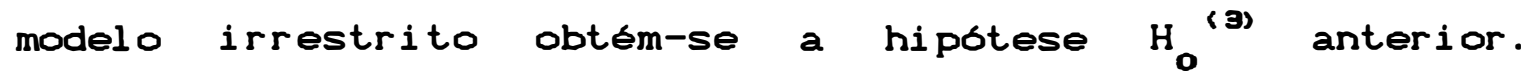
Justifica-se, desta forma, o aparecimento dos parametros $\gamma$ nessa hi pótese.

Neste caso $\mathrm{Ho}^{(3)}$ é igual a $\mathrm{Ho}^{(2)}$, e a soma de quadrados tipo III para o fator A é obtida da mesma forma.

Para obter-se as funçỡes estimáveis tipo III para o fator B (espaçamentos), procede-se da seguinte forma: - tomar a forma geral de funções estimáveis da Tabela 4 e igualar $\mathrm{L}_{1} \in \mathrm{L}_{2}$ a zero;

- obter $L_{0}$ em função de $L_{4} e L_{5}$, a fim de tornar a hipótese de A ortogonal a hipótese de $A \times B$, da seguinte forma:

$$
\begin{array}{ccccccccccc}
\mu & \alpha_{1} & \alpha_{2} & \beta_{1} \beta_{2} & \beta_{3} & \gamma_{11} & \gamma_{12} & \gamma_{13} & \gamma_{22} & \gamma_{23} \\
B- & 0 & 0 & 0 & L_{4} & L_{5}-L_{4}-L_{5} & L_{4} & L_{8}-L_{4}-L_{8} & L_{5}-L_{8}-L_{5}+L_{8} \\
A \times B-0 & 0 & 0 & 0 & 0 & 0 & 0 & L_{8} & -L_{8} & -L_{8} & L_{8}
\end{array}
$$

Multiplicando-se os coeficintes de cada parâmetro nas duas linhas, somando-se e igualando-se a zero, obtém-se

$$
\mathrm{L}_{\mathrm{\theta}}=-1 / 4 \mathrm{~L}_{4}+1 / 2 \mathrm{~L}_{5}
$$

As funçỡes estimáveis resultantes desse processo são apresentados na Tabela 16. 
94.

Tabel a 16 - Funçðes Estimaveis Tipo III para B.

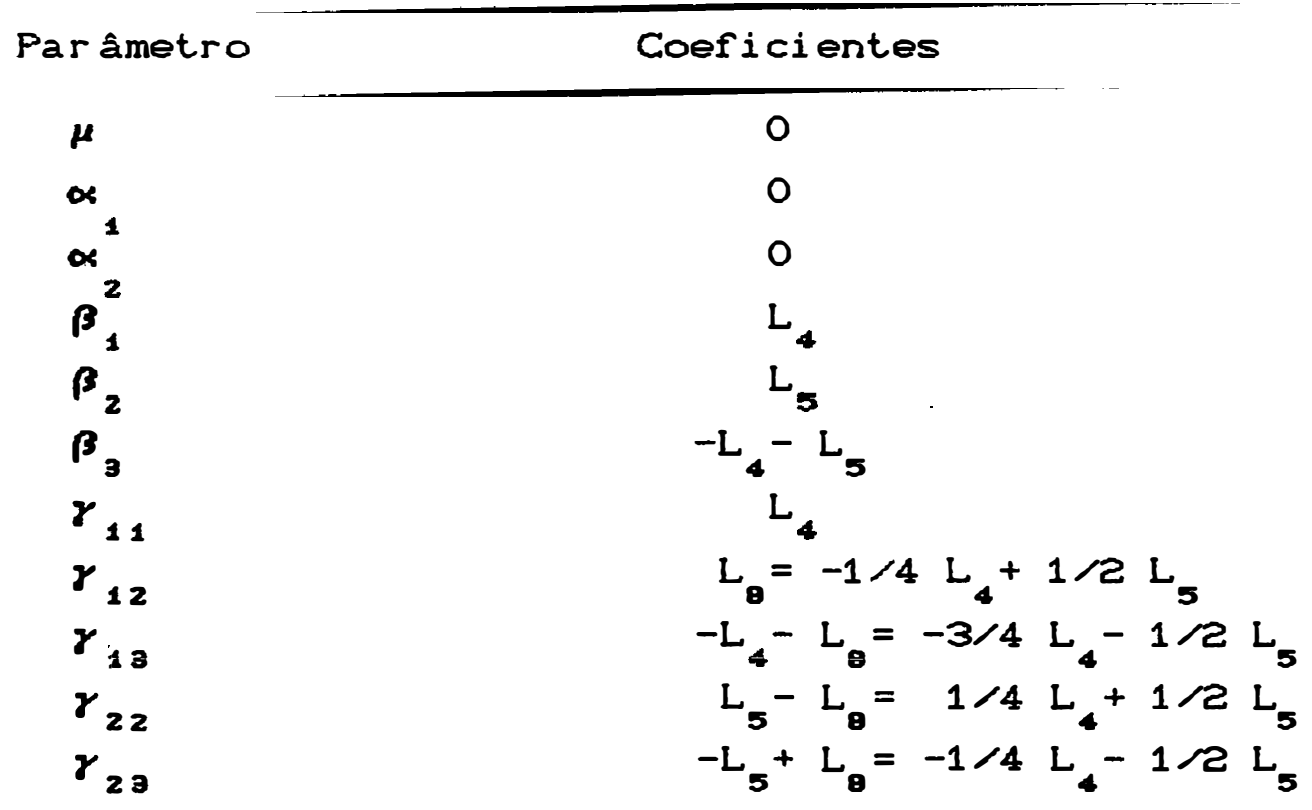

Observa-se que o coeficiente de $\gamma_{11}$ é igual ao coeficiente de $\beta_{1}$. A soma dos coeficientes de $\gamma_{12} e \gamma_{22}$ é igual ao coeficiente de $\beta_{2}$ e a soma dos coeficientes de $\boldsymbol{\gamma}_{13}$ e $\gamma_{23}$ é igual ao coeficiente de $\beta_{3}$. Também, as somas de $\boldsymbol{\gamma}_{11}$, $\gamma_{12} \operatorname{com} \gamma_{13} e \gamma_{22} \operatorname{com} \gamma_{23}$ são iguais a zero coeficientes de $\alpha_{1} e \alpha_{2}$.

Para obter-se as hipóteses tipo III para o fator $B$, atribui-se valores a $L_{4} \odot L_{5}$. Assim fazendo-se $L_{4}=$ 1. $L_{5}=0$ e depois $L_{4}=0$ e $L_{5}=1$, será obtida a hipótese

$$
H_{0}^{(0)}:\left\{\begin{array}{cc}
\beta_{1} & -\beta_{3}+\gamma_{11}-\frac{1}{4} \gamma_{12}-\frac{3}{4} \gamma_{13}+\frac{1}{4} \gamma_{22}-\frac{1}{4} \gamma_{23}=0 \\
& \beta_{2}-\beta_{3}+\frac{1}{2} \gamma_{12}-\frac{1}{2} \gamma_{13}+\frac{1}{2} \gamma_{22}-\frac{1}{2} \gamma_{23}=0
\end{array}\right.
$$

que uma hipótese sobre médias não ponderadas de col unas.

A hipotese tipo III para B equivale, em termos da notação $R C)$ a $R(\dot{\beta} \mid \dot{\mu}, \dot{\alpha}, \dot{\gamma})$. 
95.

Em termos dos parámetros do modelo restrito $H^{(0)}$ é dada por

$$
H_{0}^{(0)}: \quad \dot{\beta}_{j}=0 \quad \forall j
$$

As funçס̋es estimáveis tipo III para B podem ser obtidas de uma forma mais compreensivel através do algoritmo de SEARLE (1980), descrito no item 3.6.2, da seguinte forma:

- tomar contrastes baseados nos parámetros $\beta$ que não envol vam os parámetros $\alpha$, como estes

$\beta_{1}-\beta_{2}+\gamma_{11}-\gamma_{12}$

$\beta_{1}-\beta_{3}+\gamma_{11}-\gamma_{13}$

$\beta_{2}-\beta_{3}+1 / 2\left(\gamma_{12}+\gamma_{22}{ }^{2}-1 / 2\left(\gamma_{13}+\gamma_{23}{ }^{3}\right.\right.$

- usando coeficientes $m_{1}, m_{2} \in m_{3}$, formar uma combinação linear destes contrastes

$$
\begin{aligned}
f= & m_{1}\left[\beta_{1}-\beta_{2}+\gamma_{11}-\gamma_{12}\right]+m_{2}\left[\beta_{1}-\beta_{3}+\gamma_{11}-\gamma_{13}\right]+ \\
& +m_{3}\left[\beta_{2}-\beta_{3}+1 / 2\left(\gamma_{12}+\gamma_{22}\right]-1 / 2\left(\gamma_{13}+\gamma_{23}\right)\right]
\end{aligned}
$$

- torná-los ortogonais ao contraste dos $\gamma$ dados por $\gamma_{12}-\gamma_{13}-\gamma_{22}+\gamma_{23}$ e fazer o contraste para $\beta$ ortogonal a este. 
96.

Tabela 17 - Coeficientes m para a Combinação Linear f e para a Interação

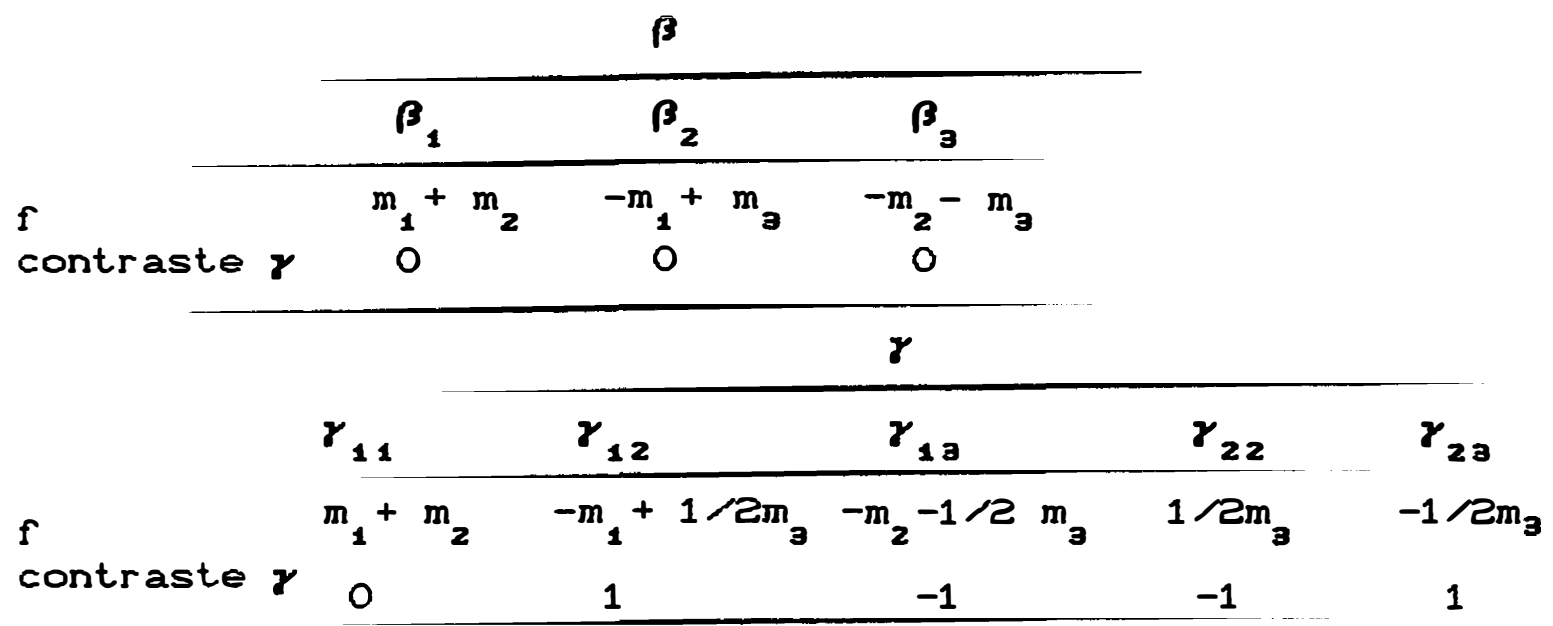

A ortogonalidade entre os dois implica $-m_{1}+1 / 2 m_{3}+m_{2}+1 / 2 m_{3}-1 / 2 m_{3}-1 / 2 m_{3}=0$

portanto $m_{1}=m_{2}$.

- o fator B tem dois graus de liberdade, então existem dois valores arbitrários para os $m, L_{4} e L_{5}$, sendo a função para $\beta_{1}$ e $\beta_{2}$ igual a $L_{4} \beta_{1}+L_{5} \beta_{2}$, então

$$
m_{1}+m_{2}=L_{4} \text { e }-m_{1}+m_{3}=L_{5}
$$

assim,

$$
m_{1}=1 / 2 L_{4} ; m_{2}=1 / 2 L_{4} \quad \text { e } m_{3}=L_{5}+1 / 2 L_{4}
$$

e substituindo em $f$

$$
\begin{aligned}
f= & 1 / 2 L_{4}\left[\beta_{1}-\beta_{2}+\gamma_{11}-\gamma_{12}\right]+1 / 2 L_{4}\left[\beta_{1}-\beta_{3}+\gamma_{11}-\gamma_{13}\right]+ \\
& +L_{5}+1 / 2 L_{4}\left[\beta_{2}-\beta_{3}+1 / 2\left[\gamma_{12}+\gamma_{22}\right]-1 / 2\left[\gamma_{13}+\gamma_{23}\right]\right] \\
f= & L_{4} \beta_{1}+L_{5} \beta_{2}+\left[-L_{4}-L_{5}\right] \beta_{3}+L_{4} \gamma_{11}+\left[-1 / 4 L_{4}+1 / 2 L_{5}\right] \gamma_{12}+ \\
& +\left[-3 / 4 L_{4}-1 / 2 L_{5}\right] \gamma_{13}+\left[1 / 4 L_{4}+1 / 2 L_{5}\right] \gamma_{22}+\left[-1 / 4 L_{4}-1 / 2 L_{5}\right] \gamma_{23}
\end{aligned}
$$


que são as funçơes estimáveis tipo III para B apresentadas na Tabela 16.

A hipótese $H_{0}^{(0)}$ em termos do Modelo de Médias de Caselas fica:

$$
H_{0}^{(\sigma)}:\left\{\begin{array}{r}
\mu_{11}-\frac{1}{4} \mu_{12}-\frac{3}{4} \mu_{13}+\frac{1}{4} \mu_{22}-\frac{1}{4} \mu_{23}=0 \\
\frac{1}{2} \mu_{12}-\frac{1}{2} \mu_{13}+\frac{1}{2} \mu_{22}-\frac{1}{2} \mu_{23}=0
\end{array}\right.
$$

A primeira linha da hipótese campara a col una um com as outras duas, na linha um $e$ as colunas dois $\theta$ trés na linha dois, envolvendo coeficientes dificeis de entender.

$$
\text { A segunda linha compara colunas um e tres }
$$
envol vendo linhas um e dois.

Para obtenção da Soma de Quadrados tipo III para o fator B, se irá calcular

$$
\begin{aligned}
& R(\dot{\beta} \mid \dot{\mu}, \dot{\alpha}, \dot{\gamma})=S Q H_{0}^{(\sigma)}=\left[B^{\prime}(\sigma\rangle^{\mu^{0}}\right]^{\prime}\left[B^{\prime}(\sigma)^{(w)} w^{-1} B_{(\sigma)}\right]^{-1}\left[B^{\prime}(\sigma)^{\mu}\right] \\
& \text { onde } B^{\prime}(\sigma)=\left[\begin{array}{ccccc}
1 & -1 / 4 & -3 / 4 & 1 / 4 & -1 / 4 \\
0 & 1 / 2 & -1 / 2 & 1 / 2 & -1 / 2
\end{array}\right] \\
& R C \dot{\beta} \mid \dot{\mu}, \dot{\alpha}, \dot{\gamma})=S Q H_{0}^{(\sigma)}=1247,315
\end{aligned}
$$

Aplicando o algoritmo de SEARLEC1980), para converter as hipóteses em termos dos parámetros do modelo 
98.

restrito para o modelo irrestrito obtém-se:

$$
\begin{gathered}
Z=\left[\begin{array}{rrrrr}
1 & 1 & 1 & 0 & 0 \\
1 & 1 & 0 & 1 & 1 \\
1 & 1 & -1 & -1 & -1 \\
1 & 1 & -1 & -1 & -1 \\
1 & -1 & 0 & 1 & -1 \\
1 & -1 & 0 & 1 & -1 \\
1 & -1 & -1 & -1 & 1
\end{array}\right] \\
W=\left[\begin{array}{rrrrrrrrrrr}
1 & 1 & 0 & 1 & 0 & 0 & 1 & 0 & 0 & 0 & 0 \\
1 & 1 & 0 & 0 & 1 & 0 & 0 & 1 & 0 & 0 & 0 \\
1 & 1 & 0 & 0 & 0 & 1 & 0 & 0 & 1 & 0 & 0 \\
1 & 0 & 1 & 0 & 1 & 0 & 0 & 0 & 0 & 1 & 0 \\
1 & 0 & 1 & 0 & 0 & 1 & 0 & 0 & 0 & 0 & 1
\end{array}\right]
\end{gathered}
$$

onde:

2 é a matriz do delineamento para o Modelo $\Sigma$

$W_{s}$ é formada pelas linhas distintas de Z

W é formada pelas linhas distintas da matriz X Cmatriz do delineamento do Modelo S, já apresentada

$$
\beta_{s}=W_{s}^{-1} W
$$

onde:

$\beta_{s}$ é o vetor de parametros do modelo restrito

B $e$ vetor de parámetros no modelo irrestrito 
99.

$$
\begin{aligned}
& {\left[\begin{array}{l}
\mu \\
\alpha_{1} \\
\beta_{1} \\
\beta_{2} \\
\beta_{12}
\end{array}\right]=\left[\begin{array}{ccccccccccc}
1 & 1 / 2 & 1 / 2 & 1 / 3 & 1 / 3 & 1 / 3 & 1 / 3 & 1 / 12 & 1 / 12 & 1 / 4 & 1 / 4 \\
0 & 1 / 2 & -1 / 2 & 0 & 0 & 0 & 0 & 1 / 4 & 1 / 4 & -1 / 4 & -1 / 4 \\
0 & 0 & 0 & 2 / 3 & -1 / 3 & -1 / 3 & 2 / 3 & -1 / 3 & -1 / 3 & 0 & 0 \\
0 & 0 & 0 & -1 / 3 & 2 / 3 & -1 / 3 & -1 / 3 & 5 / 12 & -1 / 12 & 1 / 4 & -1 / 4 \\
0 & 0 & 0 & 0 & 0 & 0 & 0 & 1 / 4 & -1 / 4 & -1 / 4 & 1 / 4
\end{array}\right]\left[\begin{array}{l}
\mu \\
\alpha_{1} \\
\alpha_{2} \\
\beta_{1} \\
\beta_{2} \\
\beta_{3} \\
\gamma_{11} \\
\gamma_{12} \\
\gamma_{12} \\
\gamma_{22} \\
\gamma_{23}
\end{array}\right]} \\
& \dot{\alpha}_{1}=\frac{1}{2} \alpha_{1}-\frac{1}{2} \alpha_{2}+\frac{1}{4} \gamma_{12}+\frac{1}{4} \gamma_{13}-\frac{1}{4} \gamma_{22}-\frac{1}{4} \gamma_{23} \\
& \dot{\beta}_{1}=\frac{2}{3} \beta_{1}-\frac{1}{3} \beta_{2}-\frac{1}{3} \beta_{3}+\frac{2}{3} \gamma_{11}-\frac{1}{3} \gamma_{12}-\frac{1}{3} \gamma_{13} \\
& \dot{\beta}_{2}=-\frac{1}{3} \beta_{1}+\frac{2}{3} \beta_{2}-\frac{1}{3} \beta_{2}-\frac{1}{3} \gamma_{11}+\frac{5}{12} \gamma_{12}-\frac{1}{12} \gamma_{12}+\frac{1}{4} \gamma_{22}-\frac{1}{4} \gamma_{2 a} \\
& \dot{\gamma}_{12}=\frac{1}{4} \gamma_{12}-\frac{1}{4} \gamma_{13}-\frac{1}{4} \gamma_{22}+\frac{1}{4} \gamma_{23}
\end{aligned}
$$

A primeira hipótese, $H_{0}^{(3)}: \dot{\alpha}_{1}=0$ a mesma obtida tomando-se as funçбes estimáveis tipo III para o fator A (Tabela 15) e substituindo-se $L_{2}$ por $1 / 2$.

A segunda hipótese $H^{(\sigma)}:\left\{\begin{array}{l}\dot{\beta}_{1}=0 \\ \hat{\beta}_{2}=0\end{array}\right.$ seria obtida tomando-se as funçőes estimáveis tipo III para B (Tabela 16) e substituindo-se primeiro $L_{4}$ por $2 / 3$ e $L_{5}$ por 
100.

$-1 / 3$ e em seguida $L_{4}$ por $-1 / 3 \in L_{5}$ por $2 / 3$

4. 1.6. Obtenção das Funções Estimáveis Tipo IV

Da mesma forma, para obter as funçశes estimáveis tipo IV para o fator A (variedades), deve-se tomar a forma geral de funçơses estimáveis apresentada na Tabela 4 e igualar $\mathrm{L}_{1}, \mathrm{~L}_{4} e \mathrm{~L}_{5}$ a zero. Em seguida:

- verifica-se que o coeficiente de $\gamma_{11}$ é zero enquanto que o coeficiente de $\alpha_{1}$ não o e. Com isto, a função estimável tipo IV para o fator A não é única;

- para os efeitos de $\gamma$ que não tem coeficiente zero conta-se quantas vezes cada nivel de ocorre em $\gamma$ e observa-se que $\alpha_{1}$ ocorre duas vezes $e \alpha_{2}$ também. Então iguala-se $L_{8} a$ $1 / \mathrm{KL}_{2}$ onde $\mathrm{K} \in$. 0 número de vezes que $\alpha_{1}$ aparece em $\gamma$ desconsiderando-se $\gamma_{11}$ que tem coeficiente zero. Portanto $\mathrm{L}_{\mathbf{8}}=1 / 2 \mathrm{~L}_{2}$.

As funçớes estimáveis tipo IV para o fator A (variedades) obtidas por este processo se encontram na Tabela 18. 
101

Tabela 18 - Funçơes Estimáveis Tipo IV para A.

\begin{tabular}{cc}
\hline Parametros & Coficientes \\
\hline$\mu_{1}$ & 0 \\
$\alpha_{2}$ & $-L_{2}$ \\
$\beta_{1}$ & 0 \\
$\beta_{2}$ & 0 \\
$\beta_{3}$ & 0 \\
$\gamma_{11}$ & 0 \\
$\gamma_{12}$ & $L_{8}=1 / 2 L_{2}$ \\
$\gamma_{13}$ & $L_{2}-L_{8}=1 / 2 L_{2}$ \\
$\gamma_{22}$ & $-L_{8}=-1 / 2 L_{2}$ \\
$\gamma_{23}$ & $-L_{2}+L_{8}=-1 / 2$ \\
\hline
\end{tabular}

Para obter-se as hipóteses tipo IV para o fator $A$, atribui-se valores à $L_{2}$. Se $L_{2}$ igual a 1 , obtém-se a hipótese

$$
H_{0}^{\langle\theta\rangle}: \alpha_{1}-\alpha_{2}+\frac{1}{2} \gamma_{12}+\frac{1}{2} \gamma_{13}-\frac{1}{2} \gamma_{22}-\frac{1}{2} \gamma_{23}=0
$$

Observa-se que para este padrão de caselas vazias, as hipóteses tipos II, III e IV para A são idénticas. Sua forma no Modelo $M$ e sua Soma de Quadrados já foram apresentadas anteriormente.

Para obtenção das funçర̃es estimáveis tipo IV para o fator B (espaçamentos), deve-se considerar a forma geral de funçớes estimáveis e igualar $L_{1}$ e $L_{2}$ a zero.

- nivel um do fator $B$ ocorre uma vez na interação AxB, o nivel dois ocorre duas vezes e o nível trés 
também.

Portanto:

- como $\beta_{1}$ só aparece em $\boldsymbol{\gamma}_{11}$, iguala-se o coeficiente de $\boldsymbol{\gamma}_{11}$ aio de $\beta_{1}$;

- $\beta_{2}$ aparece duas vezes nos parámetros $\gamma$ e por isso 0 coeficiente de $\beta_{2} C_{5} L^{2}$ será distribuido entre $\boldsymbol{\gamma}_{12}{ }^{e} \boldsymbol{\gamma}_{22}$. Tomando-se o coeficiente de $\gamma_{12} \mathrm{CL}_{8}{ }^{2}$ igual a metade do coeficiente de $\beta_{2}\left(L_{5}\right)$, obtém-se as funçơes estimáveis tipo IV para o fator B apresentadas na Tabela 19.

Tabel a 19 - Funçỡes Estimáveis Tipo IV para B.

\begin{tabular}{lc}
\hline Par ametro & Coeficientes \\
\hline$\beta_{1}$ & 0 \\
$\alpha_{1}$ & 0 \\
$\alpha_{2}$ & 0 \\
$\beta_{1}$ & $L_{4}$ \\
$\beta_{2}$ & $L_{5}$ \\
$\beta_{3}$ & $-L_{4}-L_{5}$ \\
$\gamma_{11}$ & $L_{4}=1 / 2 L_{5}$ \\
$\gamma_{12}$ & $-L_{4}-L_{8}=-L_{4}-1 / 2 L_{5}$ \\
$\gamma_{13}$ & $L_{5}-L_{8}=1 / 2 L_{5}$ \\
$\gamma_{22}$ & $-L_{5}+L_{8}=-1 / 2 L_{5}$ \\
$\gamma_{23}$ &
\end{tabular}

Tomando-se $L_{4}$ igual a $1, L_{5}$ igual a 0 e depois $L_{4}$ igual a $0 e L_{5}$ igual a 1 , obtém-se a hipótese:

$$
H_{0}^{(0)}: \begin{cases}\beta_{1}-\beta_{3}+\gamma_{11}-\gamma_{13} & =0 \\ & \beta_{2}-\beta_{3}+\frac{1}{2} \gamma_{12}-\frac{1}{2} \gamma_{13}+\frac{1}{2} \gamma_{22}-\frac{1}{2} \gamma_{23}=0\end{cases}
$$


$H^{(0)}$ constitui-se de contrastes entre médias de caselas da mesma linha, começando pela última coluna.

As hipóteses tipo IV não são construidas com base na notação RC $\supset$. Consideram um subconjunto dos dados onde não aparecem casel as vazias.

Podem ser melhor interpretadas em termos do Modelo M, tomando a forma

$$
H_{0}^{(9)}: \quad\left\{\begin{array}{cl}
\mu_{11}-\mu_{13} & =0 \\
\frac{1}{2} \mu_{12}-\frac{1}{2} \mu_{13}+\frac{1}{2} \mu_{22}-\frac{1}{2} \mu_{23} & =0
\end{array}\right.
$$

Observando-se o padrão dos dados pode-se verificar que na primeira linha da hipótese, quando se compara níveis 1 e 3 do fator $B$, so aparece nivel 1 do fator A, pois a primeira casela do nivel 2 é vazia. Já a segunda linha da hipótese considera, na comparação entre os niveis 2 e 3 do fator $B$, os dois niveis do fator $A$ pois nestes, todas as caselas são completas.

Para obtenção da Soma de Quadrados tipo IV para o fator B, calcula-se

$$
\left.\mathrm{SQH}_{0}^{(0)}=\left[\mathrm{B}^{\prime}(0)^{\mu^{0}}\right]^{\prime}\left[\mathrm{B}^{\prime}(0)^{\left(O W^{\prime}\right.} W\right)^{-1} \mathrm{~B}_{(0)}\right]^{-1}\left[\mathrm{~B}^{\prime}(0)^{\mu^{0}}\right]
$$

onde

$$
\begin{aligned}
& B_{(O)}^{\prime}=\left[\begin{array}{ccccc}
1 & 0 & -1 & 0 & 0 \\
0 & 1 / 2 & -1 / 2 & 1 / 2 & -1 / 2
\end{array}\right] \\
& \mathrm{SQH}_{0}^{(0)}=1297,853
\end{aligned}
$$


104.

\subsubsection{Consequencias da Alteraçáo da Ordem de Especificação do Modelo}

Caso as variáveis de classificação sejam especificadas no modelo, ao PROC GLM, na ordem B, A, A*B, as funçơes estimáveis do tipo I serão alteradas. A hipótese tipo I para o fator $B$ sera uma hipótese sobre médias não ajustadas, representada por $R(\beta \mid \mu)$, a qual não aparecia em nenhum tipo na ordenação $A, B, A * B$. Já a hipótese tipo I para o fator A será ajustada e expressa por $R(\alpha \mid \mu, \beta) e$ é a mesma hipótese tipo II dada pela ordenação $A, B, A * B$. A hipótese tipo I para a interação e demais hipóteses permanecem inal teradas.

Para obtenção da hipótese tipo I para o fator B, basta seguir os mesmos passos ja apresentados.

Neste caso, a partição da matriz X é feita da seguinte forma

$x_{1}=\left[\begin{array}{llll}1 & 1 & 0 & 0 \\ 1 & 0 & 1 & 0 \\ 1 & 0 & 0 & 1 \\ 1 & 0 & 0 & 1 \\ 1 & 0 & 1 & 0 \\ 1 & 0 & 1 & 0 \\ 1 & 0 & 0 & 1\end{array}\right] ; \quad x_{2}=\left[\begin{array}{ll}1 & 0 \\ 1 & 0 \\ 1 & 0 \\ 1 & 0 \\ 0 & 1 \\ 0 & 1 \\ 0 & 1\end{array}\right] ; x_{3}=\left[\begin{array}{lllll}1 & 0 & 0 & 0 & 0 \\ 0 & 1 & 0 & 0 & 0 \\ 0 & 0 & 1 & 0 & 0 \\ 0 & 0 & 1 & 0 & 0 \\ 0 & 0 & 0 & 1 & 0 \\ 0 & 0 & 0 & 1 & 0 \\ 0 & 0 & 0 & 0 & 1\end{array}\right]$

onde:

$X_{1}$ é formada pelas colunas de $X$ correspondentes a $\mu$ ao fator B; 
$X_{2}$ É formada pelas col unas de $X$ correspondentes ao fator $A$;

$\mathrm{X}_{3}$ é formada pelas colunas de $\mathrm{X}$ correspondentes à interação.

$$
\begin{gathered}
G_{1}=\left(x_{1}^{\prime} x_{1}\left|x_{1}^{\prime} x_{2}\right| x_{1} x_{3}\right)= \\
=\left[\begin{array}{llll|ll|lllll}
7 & 1 & 3 & 3 & 4 & 3 & 1 & 1 & 2 & 2 & 1 \\
1 & 1 & 0 & 0 & 1 & 0 & 1 & 0 & 0 & 0 & 0 \\
3 & 0 & 3 & 0 & 1 & 2 & 0 & 1 & 0 & 2 & 0 \\
3 & 0 & 0 & 3 & 2 & 1 & 0 & 0 & 2 & 0 & 1
\end{array}\right]
\end{gathered}
$$

e então $\quad G_{1}^{*}=\left(x_{1}^{\prime} x_{1}\right)^{-} G_{1}=$

$$
=\left[\begin{array}{cccc|cc|ccccc}
\mu & \beta_{1} & \beta_{2} & \beta_{3} & \alpha_{1} & \alpha_{2} & \gamma_{11} & \gamma_{12} & \gamma_{13} & \gamma_{22} & \gamma_{23} \\
1 & 0 & 0 & 1 & 2 / 3 & 1 / 3 & 0 & 0 & 2 / 3 & 0 & 1 / 3 \\
0 & 1 & 0 & -1 & 1 / 3 & -1 / 3 & 1 & 0 & -2 / 3 & 0 & -1 / 3 \\
0 & 0 & 1 & -1 & -1 / 3 & 1 / 3 & 0 & 1 / 3 & -2 / 3 & 2 / 3 & -1 / 3 \\
0 & 0 & 0 & 0 & 0 & 0 & 0 & 0 & 0 & 0 & 0
\end{array}\right] \begin{aligned}
& L_{1} \\
& L_{2} \\
& L_{3}
\end{aligned}
$$

$G_{1}^{*} \theta$ produz as funçőes estimáveis tipo $I$ para o fator $B$ apresentadas na Tabela 20 . 
106.

Tabel a 20 - Funçơes Estimáveis Tipo I para B. (ordem $B, A, A * B$ )

\begin{tabular}{lc}
\hline Parámetro & Coeficiente \\
\hline$\mu_{12}$ & 0 \\
$\beta_{1}$ & $\mathrm{~L}_{2}$ \\
$\beta_{2}$ & $\mathrm{~L}_{3}$ \\
$\beta_{3}$ & $-\mathrm{L}_{2}-\mathrm{L}_{3}$ \\
$\alpha_{1}$ & $1 / 3 \mathrm{~L}_{2}-1 / 3 \mathrm{~L}_{3}$ \\
$\alpha_{2}$ & $-1 / 3 \mathrm{~L}_{2}+1 / 3 \mathrm{~L}_{3}$ \\
$\gamma_{11}$ & $\mathrm{~L}_{2}$ \\
$\gamma_{12}$ & $1 / 3 \mathrm{~L}_{3}$ \\
$\gamma_{13}$ & $-2 / 3 \mathrm{~L}_{2}-2 / 3 \mathrm{~L}_{3}$ \\
$\gamma_{22}$ & $2 / 3 \mathrm{~L}_{3}$ \\
$\gamma_{23}$ & $-1 / 3 \mathrm{~L}_{2}-1 / 3 \mathrm{~L}_{3}$ \\
\hline
\end{tabular}

Fazendo-se $L_{2}=1$ e $L_{3}=0$, depois, $L_{2}=0 e$ $L_{3}=1$, obtém-se

$$
H_{0}^{(4)}:\left\{\begin{array}{l}
\beta_{1}-\beta_{3}+\frac{1}{3} \alpha_{1}-\frac{1}{3} \alpha_{2}+\gamma_{11}-\frac{2}{3} \gamma_{13}-\frac{1}{3} \gamma_{23}=0 \\
\beta_{2}-\beta_{3}-\frac{1}{3} \alpha_{1}+\frac{1}{3} \alpha_{2}+\frac{1}{3} \gamma_{12}-\frac{2}{3} \gamma_{13}+\frac{2}{3} \gamma_{22}-\frac{1}{3} \gamma_{23}=0
\end{array}\right.
$$

E uma hipótese para médias ponderadas de colunas não ajustadas para linhas e equivale a $R(\beta \mid \mu)$.

Pode ser escrita em termos do Modelo M:

$$
H 0^{(4)}:\left\{\begin{array}{l}
\mu_{11}=\frac{2 \mu_{13}+\mu_{23}}{3} \\
\frac{\mu_{12}+2 \mu_{22}}{3}=\frac{2 \mu_{13}+\mu_{23}}{3}
\end{array}\right.
$$

A Soma de Quadrados tipo I para o fator B é obtida através de 
107.

$$
\begin{aligned}
& R(\beta \mid \mu)=S Q H_{0}{ }^{(4)}=\left[B^{\prime}{ }_{(4)^{\prime}} \mu^{0}\right]^{\prime}\left[B_{\langle 4\rangle^{\prime}}\left(w^{\prime} W\right)^{-1} B_{(4)}\right]^{-1}\left[B^{\prime}(4)^{\prime} \mu^{0}\right] \\
& \text { onde } \\
& B_{(4)}^{\prime}=\left[\begin{array}{ccccc}
1 & 0 & -2 / 3 & 0 & -1 / 3 \\
0 & 1 / 3 & -2 / 3 & 2 / 3 & -1 / 3
\end{array}\right] \\
& R(\beta \mid \mu)=S Q H_{0}^{(4)}=1993,714
\end{aligned}
$$

A função estimável tipo I para o fator A, quando o modelo é especificado na ordem $B, A, A x B$ é dada por

$$
\begin{gathered}
G_{2}=C O\left|X_{2}^{\prime} M_{1} X_{2}\right| X X_{2}^{\left.M_{1} X_{3}\right)} \\
M_{1}=I-X_{1}\left(X_{1}^{\prime} X_{1}\right)^{-} X_{1}^{\prime} \\
G_{2}^{*}=\left(X_{2}^{\prime} M_{1} X_{2}\right)^{-G_{2}}
\end{gathered}
$$

- que resulta nas mesmas funçơes estimáveis apresentadas na Tabela 12, pois equivalem às funçơes estimáveis tipo II para A. 
108.

Tabela 21 - Análise de Variancia dos Dados da Tabela 6 Através do Modelo com Dois Fatores e Interação, para Ordenação A, B, AxB.

IIPO I

\begin{tabular}{lcccr}
\hline $\begin{array}{c}\text { Variaçós } \\
\text { Consideradas }\end{array}$ & $\begin{array}{c}\text { Graus de } \\
\text { Liberdade }\end{array}$ & $\begin{array}{c}\text { Hipóteses } \\
\text { Testadas }\end{array}$ & $R()$ & $\begin{array}{r}\text { Soma de } \\
\text { Quadrados }\end{array}$ \\
\hline A (nå ajust) & 1 & $H_{0}^{(1)}$ & $R(\alpha \mid \mu)$ & 855,048 \\
B (ajustado) & 2 & $H_{0}^{(5)}$ & $R(\beta \mid \mu, \alpha)$ & 1265,417 \\
AxB & 1 & $H_{0}^{(7)}$ & $R(\gamma \mid \mu, \alpha, \beta)$ & 60,750 \\
\hline
\end{tabular}

IIPO II

\begin{tabular}{llllr}
\hline A (ajustado) & 1 & $H_{0}^{(2)}$ & $R(\alpha \mid \mu, \beta)$ & 126,750 \\
$B(a j u s t a d o)$ & 2 & $H_{0}^{(5)}$ & $R(\beta \mid \mu, \alpha)$ & 1265,417 \\
$A \times B$ & 1 & $H_{0}^{(7)}$ & $R(\gamma \mid \mu, \alpha, \beta)$ & 60,750
\end{tabular}

IIPO III

$\begin{array}{lllll}A & 1 & H_{0}^{(3)} & R(\dot{\alpha} \mid \dot{\mu}, \dot{\beta}, \dot{\gamma}) & 126,750 \\ B & 2 & H^{(0)} & R C \dot{\beta} \mid \dot{\mu}, \dot{\alpha}, \dot{\gamma}) & 1247,315 \\ \text { AxB } & 1 & H^{(3)} & R(\dot{\gamma} \mid \dot{\mu}, \dot{\alpha}, \dot{\beta}) & 60,750\end{array}$

TI PO IV

$\begin{array}{llllr}A & 1^{*} & H_{0}^{(0)} & -126,750 \\ B & 2^{*} & H_{0}^{(0)} & - & 1297,853 \\ \text { AxB } & 1 & H_{0}^{(7)} & -60,750\end{array}$

* NOTA: Existem outras hipoteses testáveis tipo IV que produzem diferentes somas de quadrados. 
109.

Tabela 22 - Análise de Variáncia dos Dados da Tabela 6 Atraves do Modelo com Dois Fatores e Interação, para ordenação B, A, AxB.

\section{TIPO I}

\begin{tabular}{lccccr}
\hline $\begin{array}{c}\text { Variaçes } \\
\text { Consideradas }\end{array}$ & $\begin{array}{c}\text { Graus de } \\
\text { Liberdade }\end{array}$ & $\begin{array}{c}\text { Hi poteses } \\
\text { Testadas }\end{array}$ & $R()$ & $\begin{array}{r}\text { Soma de } \\
\text { Quadrados }\end{array}$ \\
\hline B (nå ajust) & 2 & $H_{0}^{(4)}$ & $R(\beta \mid \mu)$ & 1993,714 \\
A (ajustado) & 1 & $H_{0}^{(2)}$ & $R(\alpha \mid \mu, \beta)$ & 126,750 \\
AxB & 1 & $H_{0}^{(7)}$ & $R(\gamma \mid \mu, \alpha, \beta)$ & 60,750 \\
\hline
\end{tabular}

IIPO II

\begin{tabular}{llllr}
\hline$B$ (ajustado) & 2 & $H_{0}^{(5)}$ & $R(\beta \mid \mu, \alpha)$ & 1265,417 \\
$A$ (ajustado) & 1 & $H_{0}^{(2)}$ & $R(\alpha \mid \mu, \beta)$ & 126,750 \\
AxB & 1 & $H_{0}^{(7)}$ & $R C \gamma \mid \mu, \alpha, \beta)$ & 60,750 \\
\hline
\end{tabular}

II PO III

\begin{tabular}{lllll}
\hline$B$ & 2 & $H_{0}^{(0)}$ & $R(\dot{\beta} \mid \dot{\mu}, \dot{\alpha}, \dot{\gamma})$ & 1247,315 \\
$A$ & 1 & $H_{0}^{(3)}$ & $R(\dot{\alpha} \mid \dot{\mu}, \dot{\beta}, \dot{\gamma})$ & 126,750 \\
$A \times B$ & 1 & $H_{0}^{(8)}$ & $R(\dot{\gamma} \mid \dot{\mu}, \dot{\alpha}, \dot{\beta})$ & 60,750 \\
\hline
\end{tabular}

\section{IIPO IV}

$\begin{array}{llll}\text { B } & 2^{*} & H_{0}^{(0)} & 1297,853 \\ \text { A } & 1^{*} & H_{0}^{(8)} & -126,750 \\ \text { AxB } & 1^{*} & H_{0}^{(7)} & -60,750\end{array}$

* NOTA: Existem outras hipóteses testáveis tipo IV que produzem diferentes somas de quadrados. 


\section{2. 2- GaSO: FATORI AL 3x4 COM QUATRO GaSELAS VAZI AS}

A Tabela 23 apresenta os dados ficticios referentes a um experimento fatorial envolvendo trés espaçamentos entre plantas e quatro doses de fitohormônios para a cultivar Golden Delicious.

Tabel a 23 - Número de Frutos/Planta

\begin{tabular}{|c|c|c|c|c|}
\hline & $\underset{j=1}{\operatorname{dose}} 1$ & $\underset{j=2}{d o s e} 2$ & $\begin{array}{c}\text { dose } \\
j=3\end{array}$ & $\begin{array}{c}\text { dose } \\
j=4\end{array}$ \\
\hline$i=1$ & 52,00 & - & 63,00 & 78,00 \\
\hline esp 1 & - & - & 47,00 & - \\
\hline Total & $52,00(1)$ & - & 110,00 (2) & $78,00(1)$ \\
\hline Médi a & 52,00 & - & 55,00 & 78,00 \\
\hline$i=2$ & 67,00 & 58,00 & - & 102,00 \\
\hline esp 2 & - & - & - & 87,00 \\
\hline Total & $67,00(1)$ & $58,00(1)$ & - & $189,00(2)$ \\
\hline Média & 67,00 & 58,00 & - & 94,50 \\
\hline$i=3$ & - & 86,00 & 104,00 & - \\
\hline $\operatorname{esp} 3$ & - & 77,00 & - & - \\
\hline Total & - & $163,00(2)$ & $104,00(1)$ & - \\
\hline Médi a & - & 81,50 & 104,00 & - \\
\hline Total & 119,00 (2) & 221,00 (3) & $214,00(3)$ & $267,00(3)$ \\
\hline Médi a & 59,50 & 73,67 & 71,33 & 89,00 \\
\hline
\end{tabular}

A Tabela 24 apresenta o número de observaçỡes por casela. 
111.

Tabel a 24 - Número de Observações por Casela

\begin{tabular}{llllll}
\hline & \multicolumn{5}{c}{$j$} \\
\cline { 2 - 6 } & 1 & 2 & 3 & 4 & $n_{i .}$ \\
\hline 1 & 1 & 0 & 2 & 1 & 4 \\
2 & 1 & 1 & 0 & 2 & 4 \\
3 & 0 & 2 & 1 & 0 & 3 \\
\hline$n \cdot j$ & 2 & 3 & 3 & 3 & 11 \\
\hline
\end{tabular}

\section{2.1. Model o Linear}

- Modelo superparametrizado dado em (22) assume a caracterização

$$
y_{i j k}=\mu+\alpha_{i}+\beta_{j}+\gamma_{i j}+e_{i j k}
$$

onde:

$y_{i j k} e ́$ a observação referente ao nivel $i$ do fator A (espaçamentos), nivel $j$ do fator $B$ (doses) e k-ésima repetição;

H uma constante inerente aos dados;

$\alpha_{i} \quad$ é o efeito do nivel $i$ do fator A. (espaçamentos);

$\beta_{j} \quad e$ éfeito do nivel $j$ do fator $B$ (doses);

$\boldsymbol{\gamma}_{i j}$ é o efeito da interação entre o nivel $i$ do fator A e nivel $j$ do fator $B$;

$e_{i j k}$ é o erro aleatório associado à observação $y_{i j k}$ tal que, $e_{i j k} \cap \mathrm{N}\left(\mathrm{O}, \alpha^{2}\right)$.

$i=1,2,3$

$j=1,2,3,4$ 
$k=0, \ldots, n_{i j}$ e número de observaçós po casela

$a=3$ (total de niveis do fator $A$

$b=4$ (total de níveis do fator B)

- Modelo de médias de caselas dado por (31) que assume a caracteri zação

$$
y_{i j k}=\mu_{i j}+e_{i j k}
$$

onde:

$y_{i j k} e e_{i j k}$ tem a mesma descrição acima;

$\mu_{i j}$ é a média da população da qual foi retirada a amostra referente ao espaçamento $i$ e dose $j$.

$$
\mu^{0}=C W^{\prime} W^{-1} w^{\prime} y
$$

$$
\mu^{\circ}=\left[\begin{array}{l}
\mu_{11}^{0} \\
\mu_{13}^{0} \\
\mu_{14}^{0} \\
\mu_{21}^{0} \\
\mu_{22}^{0} \\
\mu_{24}^{0} \\
\mu_{32}^{0} \\
\mu_{33}^{0}
\end{array}\right]=\left[\begin{array}{l}
\bar{y}_{11} \\
\bar{y}_{13} \\
\bar{y}_{14} \\
\bar{y}_{21} \\
\bar{y}_{22} \\
\bar{y}_{24} \\
\bar{y}_{32} \\
\bar{y}_{33}
\end{array}\right]=\left[\begin{array}{l}
52,00 \\
55,00 \\
78,00 \\
67,00 \\
58,00 \\
94,50 \\
81,50 \\
104,00
\end{array}\right]
$$

\subsubsection{Obtenção da Forma Geral de Funçōes Estimáveis}

Considerando-se o modelo superparametrizado $Y=X \boldsymbol{\theta}+e$, onde: 


$$
X=\left[\begin{array}{llllllllllllllll}
1 & 1 & 0 & 0 & 1 & 0 & 0 & 0 & 1 & 0 & 0 & 0 & 0 & 0 & 0 & 0 \\
1 & 1 & 0 & 0 & 0 & 0 & 1 & 0 & 0 & 1 & 0 & 0 & 0 & 0 & 0 & 0 \\
1 & 1 & 0 & 0 & 0 & 0 & 1 & 0 & 0 & 1 & 0 & 0 & 0 & 0 & 0 & 0 \\
1 & 1 & 0 & 0 & 0 & 0 & 0 & 1 & 0 & 0 & 1 & 0 & 0 & 0 & 0 & 0 \\
1 & 0 & 1 & 0 & 1 & 0 & 0 & 0 & 0 & 0 & 0 & 1 & 0 & 0 & 0 & 0 \\
1 & 0 & 1 & 0 & 0 & 1 & 0 & 0 & 0 & 0 & 0 & 0 & 1 & 0 & 0 & 0 \\
1 & 0 & 1 & 0 & 0 & 0 & 0 & 1 & 0 & 0 & 0 & 0 & 0 & 1 & 0 & 0 \\
1 & 0 & 1 & 0 & 0 & 0 & 0 & 1 & 0 & 0 & 0 & 0 & 0 & 1 & 0 & 0 \\
1 & 0 & 0 & 1 & 0 & 1 & 0 & 0 & 0 & 0 & 0 & 0 & 0 & 0 & 1 & 0 \\
1 & 0 & 0 & 1 & 0 & 1 & 0 & 0 & 0 & 0 & 0 & 0 & 0 & 0 & 1 & 0 \\
1 & 0 & 0 & 1 & 0 & 0 & 1 & 0 & 0 & 0 & 0 & 0 & 0 & 0 & 0 & 1
\end{array}\right]
$$

$$
(X, X)=\left[\begin{array}{llllllllllllllll}
11 & 4 & 4 & 3 & 2 & 3 & 3 & 3 & 1 & 2 & 1 & 1 & 1 & 2 & 2 & 1 \\
4 & 4 & 0 & 0 & 1 & 0 & 2 & 1 & 1 & 2 & 1 & 0 & 0 & 0 & 0 & 0 \\
4 & 0 & 4 & 0 & 1 & 1 & 0 & 2 & 0 & 0 & 0 & 1 & 1 & 2 & 0 & 0 \\
3 & 0 & 0 & 3 & 0 & 2 & 1 & 0 & 0 & 0 & 0 & 0 & 0 & 0 & 2 & 1 \\
2 & 1 & 1 & 0 & 2 & 0 & 0 & 0 & 1 & 0 & 0 & 1 & 0 & 0 & 0 & 0 \\
3 & 0 & 1 & 2 & 0 & 3 & 0 & 0 & 0 & 0 & 0 & 0 & 1 & 0 & 2 & 0 \\
3 & 2 & 0 & 1 & 0 & 0 & 3 & 0 & 0 & 2 & 0 & 0 & 0 & 0 & 0 & 1 \\
3 & 1 & 2 & 0 & 0 & 0 & 0 & 3 & 0 & 0 & 1 & 0 & 0 & 2 & 0 & 0 \\
1 & 1 & 0 & 0 & 1 & 0 & 0 & 0 & 1 & 0 & 0 & 0 & 0 & 0 & 0 & 0 \\
2 & 2 & 0 & 0 & 0 & 0 & 2 & 0 & 0 & 2 & 0 & 0 & 0 & 0 & 0 & 0 \\
1 & 1 & 0 & 0 & 0 & 0 & 0 & 1 & 0 & 0 & 1 & 0 & 0 & 0 & 0 & 0 \\
1 & 0 & 1 & 0 & 1 & 0 & 0 & 0 & 0 & 0 & 0 & 1 & 0 & 0 & 0 & 0 \\
1 & 0 & 1 & 0 & 0 & 1 & 0 & 0 & 0 & 0 & 0 & 0 & 1 & 0 & 0 & 0 \\
2 & 0 & 2 & 0 & 0 & 0 & 0 & 2 & 0 & 0 & 0 & 0 & 0 & 2 & 0 & 0 \\
2 & 0 & 0 & 2 & 0 & 2 & 0 & 0 & 0 & 0 & 0 & 0 & 0 & 0 & 2 & 0 \\
1 & 0 & 0 & 1 & 0 & 0 & 1 & 0 & 0 & 0 & 0 & 0 & 0 & 0 & 0 & 1
\end{array}\right]
$$

A matriz $x_{1}$, formada pelas colunas linearmente independentes, sequencialmente, da matriz $\mathrm{x} e ́$ dada por:

$$
x_{1}=\left[\begin{array}{llllllll}
1 & 1 & 0 & 1 & 0 & 0 & 1 & 0 \\
1 & 1 & 0 & 0 & 0 & 1 & 0 & 1 \\
1 & 1 & 0 & 0 & 0 & 1 & 0 & 1 \\
1 & 1 & 0 & 0 & 0 & 0 & 0 & 0 \\
1 & 0 & 1 & 1 & 0 & 0 & 0 & 0 \\
1 & 0 & 1 & 0 & 1 & 0 & 0 & 0 \\
1 & 0 & 1 & 0 & 0 & 0 & 0 & 0 \\
1 & 0 & 1 & 0 & 0 & 0 & 0 & 0 \\
1 & 0 & 0 & 0 & 1 & 0 & 0 & 0 \\
1 & 0 & 0 & 0 & 1 & 0 & 0 & 0 \\
1 & 0 & 0 & 0 & 0 & 1 & 0 & 0
\end{array}\right]
$$


114.

E a matriz $\left(X^{\prime} X\right)^{a 2}$ é construida colocando-se as colunas da matriz $\left(\mathrm{X}_{1}^{\prime} \mathrm{X}_{1}\right)^{-1}$ em lugar das colunas linearmente independentes e completando com zeros em lugar das demais colunas.

$(x \times)=\left[\begin{array}{cccccccccccccccc}2 & -2 & -3 / 2 & 0 & -1 / 2 & -3 / 2 & -2 & 0 & 1 / 2 & 2 & 0 & 0 & 0 & 0 & 0 & 0 \\ -2 & 3 & 3 / 2 & 0 & 1 / 2 & 3 / 2 & 2 & 0 & -3 / 2 & -3 & 0 & 0 & 0 & 0 & 0 & 0 \\ 3 / 2 & 3 / 2 & 3 / 2 & 0 & 0 & 1 & 3 / 2 & 0 & 0 & -3 / 2 & 0 & 0 & 0 & 0 & 0 & 0 \\ 0 & 0 & 0 & 0 & 0 & 0 & 0 & 0 & 0 & 0 & 0 & 0 & 0 & 0 & 0 & 0 \\ -1 / 2 & 1 / 2 & 0 & 0 & 3 / 2 & 1 / 2 & 1 / 2 & 0 & -3 / 2 & -1 / 2 & 0 & 0 & 0 & 0 & 0 & 0 \\ -3 / 2 & 3 / 2 & 1 & 0 & 1 / 2 & 3 / 2 & 3 / 2 & 0 & -1 / 2 & -3 / 2 & 0 & 0 & 0 & 0 & 0 & 0 \\ -2 & 2 & 3 / 2 & 0 & 1 / 2 & 3 / 2 & 3 & 0 & -1 / 2 & -3 & 0 & 0 & 0 & 0 & 0 & 0 \\ 0 & 0 & 0 & 0 & 0 & 0 & 0 & 0 & 0 & 0 & 0 & 0 & 0 & 0 & 0 & 0 \\ 1 / 2 & -3 / 2 & 0 & 0 & -3 / 2 & -1 / 2 & -1 / 2 & 0 & 7 / 2 & 3 / 2 & 0 & 0 & 0 & 0 & 0 & 0 \\ 2 & -3 & -3 / 2 & 0 & -1 / 2 & -3 / 2 & -3 & 0 & 3 / 2 & 0 / 2 & 0 & 0 & 0 & 0 & 0 & 0 \\ 0 & 0 & 0 & 0 & 0 & 0 & 0 & 0 & 0 & 0 & 0 & 0 & 0 & 0 & 0 & 0 \\ 0 & 0 & 0 & 0 & 0 & 0 & 0 & 0 & 0 & 0 & 0 & 0 & 0 & 0 & 0 & 0 \\ 0 & 0 & 0 & 0 & 0 & 0 & 0 & 0 & 0 & 0 & 0 & 0 & 0 & 0 & 0 & 0 \\ 0 & 0 & 0 & 0 & 0 & 0 & 0 & 0 & 0 & 0 & 0 & 0 & 0 & 0 & 0 & 0 \\ 0 & 0 & 0 & 0 & 0 & 0 & 0 & 0 & 0 & 0 & 0 & 0 & 0 & 0 & 0 & 0 \\ 0 & 0 & 0 & 0 & 0 & 0 & 0 & 0 & 0 & 0 & 0 & 0 & 0 & 0 & 0 & 0\end{array}\right]$

$L=(X, X)^{62}(x, X)$

$$
L=\left[\begin{array}{rrrrrrrrrrrrrrrr}
1 & 0 & 0 & 1 & 0 & 0 & 0 & 1 & 0 & 0 & 0 & 0 & -1 & 1 & 1 & 0 \\
0 & 1 & 0 & -1 & 0 & 0 & 0 & 0 & 0 & 0 & 1 & 0 & 1 & -1 & -1 & 0 \\
0 & 0 & 1 & -1 & 0 & 0 & 0 & 0 & 0 & 0 & 0 & 0 & 1 & 0 & -1 & 0 \\
0 & 0 & 0 & 0 & 0 & 0 & 0 & 0 & 0 & 0 & 0 & 0 & 0 & 0 & 0 & 0 \\
0 & 0 & 0 & 0 & 1 & 0 & 0 & -1 & 0 & 0 & 0 & 1 & 0 & -1 & 0 & 0 \\
0 & 0 & 0 & 0 & 0 & 1 & 0 & -1 & 0 & 0 & 0 & 0 & 1 & -1 & 0 & 0 \\
0 & 0 & 0 & 0 & 0 & 0 & 1 & -1 & 0 & 0 & 0 & 0 & 1 & -1 & -1 & 1 \\
0 & 0 & 0 & 0 & 0 & 0 & 0 & 0 & 0 & 0 & 0 & 0 & 0 & 0 & 0 & 0 \\
0 & 0 & 0 & 0 & 0 & 0 & 0 & 0 & 1 & 0 & -1 & -1 & 0 & 1 & 0 & 0 \\
0 & 0 & 0 & 0 & 0 & 0 & 0 & 0 & 0 & 1 & -1 & 0 & -1 & 1 & 1 & -1 \\
0 & 0 & 0 & 0 & 0 & 0 & 0 & 0 & 0 & 0 & 0 & 0 & 0 & 0 & 0 & 0 \\
0 & 0 & 0 & 0 & 0 & 0 & 0 & 0 & 0 & 0 & 0 & 0 & 0 & 0 & 0 & 0 \\
0 & 0 & 0 & 0 & 0 & 0 & 0 & 0 & 0 & 0 & 0 & 0 & 0 & 0 & 0 & 0 \\
0 & 0 & 0 & 0 & 0 & 0 & 0 & 0 & 0 & 0 & 0 & 0 & 0 & 0 & 0 & 0 \\
0 & 0 & 0 & 0 & 0 & 0 & 0 & 0 & 0 & 0 & 0 & 0 & 0 & 0 & 0 & 0 \\
0 & 0 & 0 & 0 & 0 & 0 & 0 & 0 & 0 & 0 & 0 & 0 & 0 & 0 & 0 & 0
\end{array}\right] L_{3} L_{1} L_{1}
$$

Atraves de Le obtém-se a forma geral de funções estimáveis para o exemplo apresentado na Tabela 25. 
115.

Tabela 25 - Forma Geral de Funções Estimáveis para os Dados do Exemplo da Tabela 23.

\begin{tabular}{ll}
\hline Parametros & Coeficientes \\
\hline$\alpha_{1}$ & $L_{1}$ \\
$\alpha_{2}$ & $L_{2}$ \\
$\alpha_{3}$ & $L_{3}$ \\
$\beta_{1}$ & $L_{1}-L_{2}-L_{3}$ \\
$\beta_{2}$ & $L_{5}$ \\
$\beta_{3}$ & $L_{6}$ \\
$\beta_{4}$ & $L_{7}$ \\
$\gamma_{11}$ & $L_{1}-L_{5}-L_{6}-L_{7}$ \\
$\gamma_{13}$ & $L_{0}$ \\
$\gamma_{14}$ & $L_{10}$ \\
$\gamma_{21}$ & $L_{2}-L_{0}-L_{10}$ \\
$\gamma_{22}$ & $L_{5}-L_{0}$ \\
$\gamma_{24}$ & $-L_{1}+L_{2}+L_{3}+L_{6}+L_{7}-L_{10}$ \\
$\gamma_{32}$ & $L_{1}-L_{2}-L_{5}-L_{6}-L_{7}+L_{0}+L_{10}$ \\
$\gamma_{33}$ & $L_{1}-L_{2}-L_{3}-L_{7}+L_{10}$ \\
\end{tabular}

Como oito simbolos L $\mathrm{CL}_{1}, \mathrm{~L}_{2}, \mathrm{~L}_{3}, \mathrm{~L}_{5}, \mathrm{~L}_{6}, \mathrm{~L}_{7}$, $\mathrm{L}_{\rho} \mathrm{L}_{10} \mathrm{~J}^{\mathrm{a}}$ so envolvidos, o posto máximo da hipótese tem oito graus de liberdade.

A partir desta forma geral poderão ser obtidas alguns dos tipos de funçơes estimaveis fornecidas pelo SAS.

4. 2.3. Obtenção das Funções Estimáveis Tipo I

Inicia-se a construção das funçర̃es estimáveis 
tipo I com a seguinte partição na matriz $\mathrm{X}$ :

$$
\begin{aligned}
& X_{1}=\left[\begin{array}{llll}
1 & 1 & 0 & 0 \\
1 & 1 & 0 & 0 \\
1 & 1 & 0 & 0 \\
1 & 1 & 0 & 0 \\
1 & 0 & 1 & 0 \\
1 & 0 & 1 & 0 \\
1 & 0 & 1 & 0 \\
1 & 0 & 1 & 0 \\
1 & 0 & 0 & 1 \\
1 & 0 & 0 & 1 \\
1 & 0 & 0 & 1
\end{array}\right] \quad X_{2}=\left[\begin{array}{llll}
1 & 0 & 0 & 0 \\
0 & 0 & 1 & 0 \\
0 & 0 & 1 & 0 \\
0 & 0 & 0 & 1 \\
1 & 0 & 0 & 0 \\
0 & 1 & 0 & 0 \\
0 & 0 & 0 & 1 \\
0 & 0 & 0 & 1 \\
0 & 1 & 0 & 0 \\
0 & 1 & 0 & 0 \\
0 & 0 & 1 & 0
\end{array}\right] \\
& X_{3}=\left[\begin{array}{llllllll}
1 & 0 & 0 & 0 & 0 & 0 & 0 & 0 \\
0 & 1 & 0 & 0 & 0 & 0 & 0 & 0 \\
0 & 1 & 0 & 0 & 0 & 0 & 0 & 0 \\
0 & 0 & 1 & 0 & 0 & 0 & 0 & 0 \\
0 & 0 & 0 & 1 & 0 & 0 & 0 & 0 \\
0 & 0 & 0 & 0 & 1 & 0 & 0 & 0 \\
0 & 0 & 0 & 0 & 0 & 1 & 0 & 0 \\
0 & 0 & 0 & 0 & 0 & 1 & 0 & 0 \\
0 & 0 & 0 & 0 & 0 & 0 & 1 & 0 \\
0 & 0 & 0 & 0 & 0 & 0 & 1 & 0 \\
0 & 0 & 0 & 0 & 0 & 0 & 0 & 1
\end{array}\right]
\end{aligned}
$$

onde:

$X_{1}$ é formada pelas colunas da matriz $X$ correspondentes a $\mu$ e ao fator A;

$X_{2}$ é formada pelas col unas de $X$ correspondentes ao fator $B$;

$X_{3}$ é formada pela colunas de $X$ correspondentes à interação AxB.

Para se obter as funçzes estimáveis tipo I para o fator A (espaçamentos), se irá partir da seguinte matriz: 
117.

$$
\begin{aligned}
& G_{1}=\left[x_{1}^{\prime} x_{1}\left|x_{1}^{\prime} x_{2}\right| x_{1}^{\prime} x_{3}\right]= \\
& =\left[\begin{array}{llll|llll|llllllll}
11 & 4 & 4 & 3 & 2 & 3 & 3 & 3 & 1 & 2 & 1 & 1 & 1 & 2 & 2 & 1 \\
4 & 4 & 0 & 0 & 1 & 0 & 2 & 1 & 1 & 2 & 1 & 0 & 0 & 0 & 0 & 0 \\
4 & 0 & 4 & 0 & 1 & 1 & 0 & 2 & 0 & 0 & 0 & 1 & 1 & 2 & 0 & 0 \\
4 & 0 & 0 & 4 & 0 & 2 & 1 & 0 & 0 & 0 & 0 & 0 & 0 & 0 & 2 & 1
\end{array}\right] \\
& G_{1}^{*}=\left(X_{1}^{\prime} x_{1}\right)^{-} G_{1}= \\
& =\left[\begin{array}{cccc|cccc|cccccccc}
1 & 0 & 0 & 1 & 0 & 2 / 3 & 1 / 3 & 0 & 0 & 0 & 0 & 0 & 0 & 0 & 2 / 3 & 1 / 3 \\
0 & 1 & 0 & -1 & 1 / 4 & -2 / 3 & 1 / 8 & 1 / 4 & 1 / 4 & 1 / 2 & 1 / 4 & 0 & 0 & 0 & -2 / 3 & -1 / 3 \\
0 & 0 & 1 & -1 & 1 / 4 & -5 / 12 & -1 / 3 & 1 / 2 & 0 & 0 & 0 & 1 / 4 & 1 / 4 & 1 / 2 & -2 / 3 & -1 / 3 \\
0 & 0 & 0 & 0 & 0 & 0 & 0 & 0 & 0 & 0 & 0 & 0 & 0 & 0 & 0 & 0
\end{array}\right] \begin{array}{l}
L_{1} \\
L_{2} \\
L_{3}
\end{array}
\end{aligned}
$$

A soma de quadrados tipo I para o fator A corresponde a $R(\alpha \mid \mu)$. Em vista disto, para obter-se a funçăo estimavel tipo I para A deve-se tomar a primeira linha de $G_{1}^{*}$ como zero $\mathrm{CL}_{1}$ corresponde a $\mu$.

Através de $G_{1}^{*} \theta$ obtém-se as funçס̋es estimáveis apresentadas na Tabela 26. 
Tabel a 26 - Funçðes Estimáveis Tipo I para A.

\begin{tabular}{lc}
\hline Parametros & Coeficientes \\
\hline$\alpha_{1}$ & 0 \\
$\alpha_{2}$ & $\mathrm{~L}_{2}$ \\
$\alpha_{3}$ & $-\mathrm{L}_{2}-\mathrm{L}_{3}$ \\
$\beta_{1}$ & $1 / 4 \mathrm{~L}_{2}+1 / 4 \mathrm{~L}_{3}$ \\
$\beta_{2}$ & $-2 / 3 \mathrm{~L}_{2}-5 / 12 \mathrm{~L}_{3}$ \\
$\beta_{3}$ & $1 / 6 \mathrm{~L}_{2}-1 / 3 \mathrm{~L}_{3}$ \\
$\beta_{4}$ & $1 / 4 \mathrm{~L}_{2}+1 / 2 \mathrm{~L}_{3}$ \\
$\gamma_{11}$ & $1 / 4 \mathrm{~L}_{2}$ \\
$\gamma_{13}$ & $1 / 2 \mathrm{~L}_{2}$ \\
$\gamma_{14}$ & $1 / 4 \mathrm{~L}_{2}$ \\
$\gamma_{21}$ & $1 / 4 \mathrm{~L}_{3}$ \\
$\gamma_{22}$ & $1 / 4 \mathrm{~L}_{3}$ \\
$\gamma_{24}$ & $1 / 2 \mathrm{~L}_{3}$ \\
$\gamma_{32}$ & $-2 / 3 \mathrm{~L}_{2}-2 / 3 \mathrm{~L}_{3}$ \\
$\gamma_{33}$ & $-1 / 3 \mathrm{~L}_{2}-1 / 3 \mathrm{~L}_{3}$ \\
\hline
\end{tabular}

Atribuindo-se valores a $\mathrm{L}_{2}$ e $\mathrm{L}_{3}$ obtém-se as hipóteses tipo I para o fator A.

$$
\text { Fazendo-se } \mathrm{L}_{2}=1, \mathrm{~L}_{3}=0 \text {, depois } \mathrm{L}_{2}=0 \text { e } \mathrm{L}_{3}=
$$

1, obtém-se:

$$
H_{0}^{(1)}:\left\{\begin{aligned}
\alpha_{1}-\alpha_{3}+1 / 4 \beta_{1}-2 / 3 \beta_{2}+1 / \beta \beta_{3}+1 / 4 \beta_{1}+1 / 4 \gamma_{11}+ \\
+1 / 2 \gamma_{13}+1 / 4 \gamma_{14}-2 / 3 \gamma_{32}-1 / 3 \gamma_{3 a}=0 \\
\alpha_{2}-\alpha_{3}+1 / 4 \beta_{1}-5 / 12 \beta_{2}-1 / 3 \beta_{3}+1 / 2 \beta_{1}+1 / 4 \gamma_{21}+ \\
+1 / 4 \gamma_{22}+1 / 2 \gamma_{24}-2 / 3 \gamma_{32}-1 / 3 \gamma_{3 a}=0
\end{aligned}\right.
$$

Que é uma hipótese sobre médias ponderadas de i inhas não ajustadas para colunas. Equivalente a $R(\alpha \mid \mu) \theta \theta$ 
de dificil interpretação, pois além dos parámetros $\gamma$ aparecem os parámetrosß, tornando-se praticamente impossivel visualizar o que está sendo testado.

Esta hipótese tem dois graus de liberdade $(a-1=3-1=2)$ e por isso dois símbolos $L C_{2}$ \& $\left.L_{3}\right)$ a ela associados.

Em termos dos parámetros do Modelo $M$ a hipotese fica

$$
H_{0}(1):\left\{\begin{array}{l}
\frac{1}{4} \mu_{11}+\frac{1}{2} \mu_{13}+\frac{1}{4} \mu_{14}=\frac{2}{3} \mu_{32}+\frac{1}{3} \mu_{33} \\
\frac{1}{4} \mu_{21}+\frac{1}{4} \mu_{22}+\frac{1}{2} \mu_{24}=\frac{2}{3} \mu_{32}+\frac{1}{3} \mu_{33}
\end{array}\right.
$$

que continua de dificil interpretação devido à presença dos coeficientes multiplicando os parámetros $\mu$.

Para obter-se a Soma de Quadrados tipo I para - fator A (espaçamentos), calcula-se:

$$
R(\alpha \mid \mu)=S Q H_{0}^{(1)}=\left[B_{(1)^{\prime}}^{\prime} \mu^{0}\right]^{\prime}\left[B_{(1)}^{\prime}\left(W^{\prime} w^{-1} B_{(1)}\right]^{-1}\left[B_{(1)^{\prime}}^{\prime} \mu^{0}\right]\right.
$$

onde

$$
\begin{aligned}
& B_{(1)}^{\prime}= {\left[\begin{array}{cccccccc}
\frac{1}{4} & \frac{1}{2} & \frac{1}{4} & 0 & 0 & 0 & -\frac{2}{3} & -\frac{1}{3} \\
0 & 0 & 0 & \frac{1}{4} & \frac{1}{4} & \frac{1}{2} & -\frac{2}{3} & -\frac{1}{3}
\end{array}\right] } \\
& R(\alpha \mid \mu)=S Q H_{0}{ }^{(1)}=1535,545
\end{aligned}
$$

Prosseguindo com a obtenção das funçZ̃es estimáveis tipo I para o fator B (doses), adota-se o seguinte gerador:

$$
G_{2}=C O\left|X_{2}^{\prime} M_{1} X_{2}\right| X_{2}^{\prime} M_{1} X_{3}^{2}
$$


120.

$$
\begin{aligned}
& M_{1}=I-X_{1}\left(X_{1} x_{1}\right)^{-} X_{1}^{\prime} \\
& M_{1}=\left[\begin{array}{ccccccccccc}
3 / 4 & -1 / 4 & -1 / 4 & -1 / 4 & 0 & 0 & 0 & 0 & 0 & 0 & 0 \\
-1 / 4 & 3 / 4 & -1 / 4 & -1 / 4 & 0 & 0 & 0 & 0 & 0 & 0 & 0 \\
-1 / 4 & -1 / 4 & 3 / 4 & -1 / 4 & 0 & 0 & 0 & 0 & 0 & 0 & 0 \\
-1 / 4 & -1 / 4 & -1 / 4 & 3 / 4 & 0 & 0 & 0 & 0 & 0 & 0 & 0 \\
0 & 0 & 0 & 0 & 3 / 4 & -1 / 4 & -1 / 4 & -1 / 4 & 0 & 0 & 0 \\
0 & 0 & 0 & 0 & -1 / 4 & 3 / 4 & -1 / 4 & -1 / 4 & 0 & 0 & 0 \\
0 & 0 & 0 & 0 & -1 / 4 & -1 / 4 & 3 / 4 & -1 / 4 & 0 & 0 & 0 \\
0 & 0 & 0 & 0 & -1 / 4 & -1 / 4 & -1 / 4 & 3 / 4 & 0 & 0 & 0 \\
0 & 0 & 0 & 0 & 0 & 0 & 0 & 0 & 2 / 3 & -1 / 3 & -1 / 3 \\
0 & 0 & 0 & 0 & 0 & 0 & 0 & 0 & -1 / 3 & 2 / 3 & -1 / 3 \\
0 & 0 & 0 & 0 & 0 & 0 & 0 & 0 & -1 / 3 & -1 / 3 & 2 / 3
\end{array}\right] \\
& G_{2}=\left[\begin{array}{llll|llll|cccccccc}
0 & 0 & 0 & 0 & 3 / 2 & -1 / 4 & -1 / 2 & -3 / 4 & 3 / 4 & -1 / 2 & -1 / 4 & 3 / 4 & -1 / 4 & -1 / 2 & 0 & 0 \\
0 & 0 & 0 & 0 & -1 / 4 & 17 / 12 & -2 / 3 & -1 / 2 & 0 & 0 & 0 & -1 / 4 & 3 / 2 & -1 / 2 & 2 / 3 & -2 / 3 \\
0 & 0 & 0 & 0 & -1 / 2 & -2 / 3 & 5 / 3 & -1 / 2 & -1 / 2 & 1 & -1 / 2 & 0 & 0 & 0 & -2 / 3 & 2 / 3 \\
0 & 0 & 0 & 0 & -3 / 2 & -1 / 2 & -1 / 2 & 7 / 4 & -1 / 4 & -1 / 2 & 3 / 4 & -1 / 2 & -1 / 2 & 1 & 0 & 0
\end{array}\right] \\
& G_{2}^{*}=\left(X_{2}^{\prime} M_{1} X_{2}\right)^{-} G_{2}= \\
& =\left[\begin{array}{cccc|cccc|cccccccc}
0 & 0 & 0 & 0 & 0 & 0 & -1 & 4 / 9 & -1 / 27 & -11 / 27 & 5 / 8 & 1 / 27 & -16 / 27 & -1 / 27 & 1 / 27 \\
0 & 0 & 0 & 0 & 0 & 1 & 0 & -1 & 0 & 1 / 3 & -1 / 3 & 0 & 2 / 3 & -2 / 3 & 1 / 3 & -1 / 3 \\
0 & 0 & 0 & 0 & 0 & 0 & 1 & -1 & -1 / 8 & 13 / 18 & -5 / 9 & 1 / 8 & 5 / 18 & -4 / 9 & -5 / 18 & 5 / 18 \\
0 & 0 & 0 & 0 & 0 & 0 & 0 & 0 & 0 & 0 & 0 & 0 & 0 & 0 & 0 & 0
\end{array}\right]_{7}^{L_{3}}
\end{aligned}
$$$$
\text { Atraves de } G_{2}^{*} \theta \text { obtém-se as funçன̋es estimáveis }
$$$$
\text { tipo I para o fator B (doses) apresentadas na Tabela } 27 .
$$ 
Tabela 27 - Funçб̋es Estimáveis Tipo I para B.

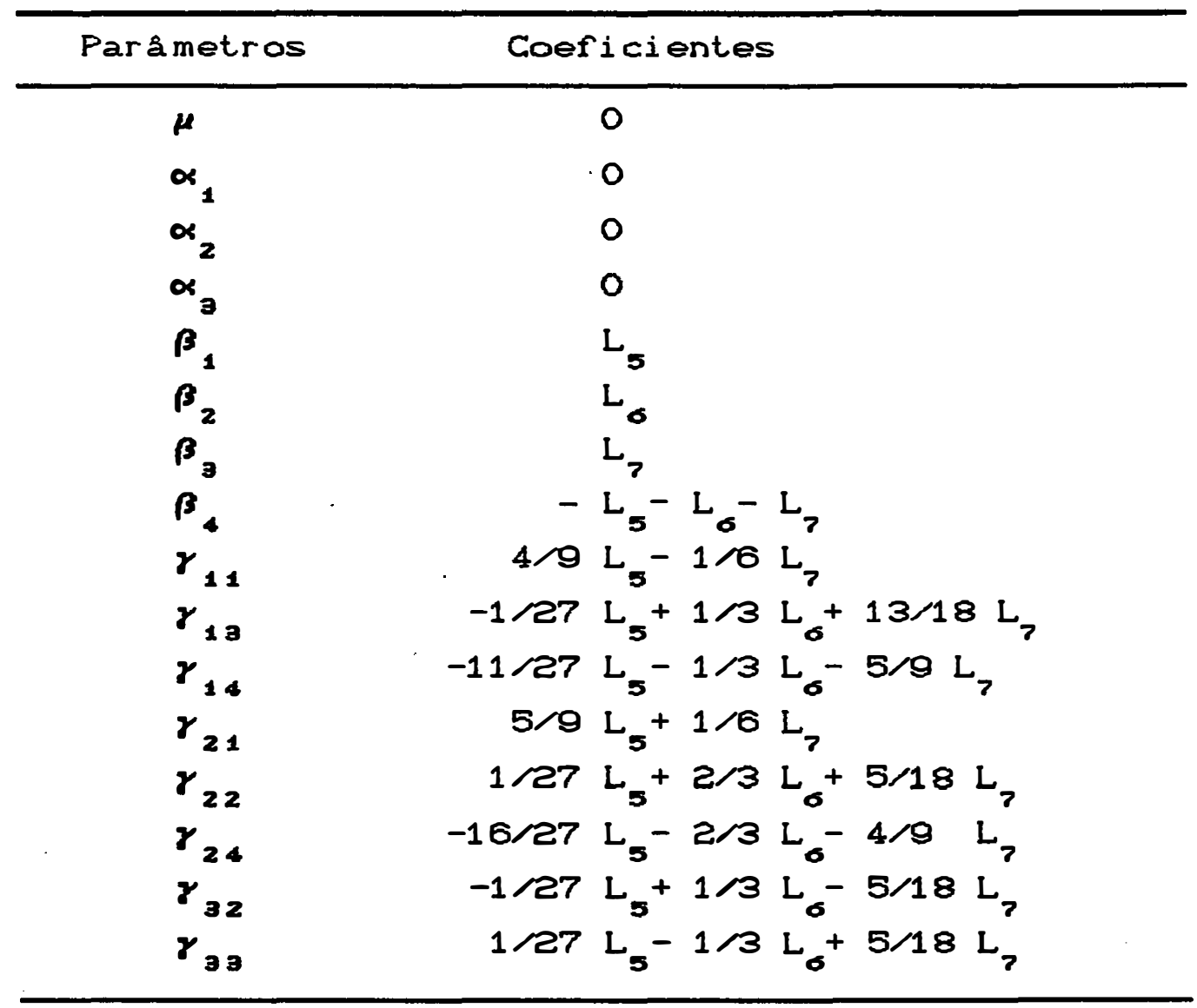

Para obter-se a hipótese tipo I para B, faz-se $L_{5}=1, L_{6}=0$ e $L_{7}=0 ; L_{5}=0, L_{6}=1$ e $L_{7}=0 ; L_{5}=0$, $L_{\sigma}=0 \ominus L_{7}=1$.

$$
H_{0}^{(5)}:\left\{\begin{array}{r}
\beta_{1}-\beta_{4}+\frac{4}{9} \gamma_{11}-\frac{1}{27} \gamma_{13}-\frac{11}{27} \gamma_{14}+\frac{5}{3} \gamma_{21}+\frac{1}{27} \gamma_{22}-\frac{16}{27} \gamma_{24}- \\
\beta_{2}-\beta_{4}+\frac{1}{3} \gamma_{13}-\frac{1}{3} \gamma_{14}+\frac{2}{3} \gamma_{22}-\frac{2}{3} \gamma_{24}+\frac{1}{3} \gamma_{32}-\frac{1}{3} \gamma_{33}=0 \\
\beta_{3}-\beta_{4}-\frac{1}{5} \gamma_{11}+\frac{13}{18} \gamma_{13}-\frac{5}{9} \gamma_{14}+\frac{1}{5} \gamma_{21}+\frac{5}{18} \gamma_{22}-\frac{4}{9} \gamma_{24}- \\
-\frac{5}{18} \gamma_{32}+\frac{5}{18} \gamma_{33}=0
\end{array}\right.
$$


que é uma hipótese sobre médias ponderadas de colunas ajustadas para linhas.

A hipótese tipo I para o fator $B$ não envolve - parámetro $\propto$. A somas de quadrados tipo I tém ajuste sequencial e por isso a SQ para o fator $B$ equivale a $R(\beta \mid \mu, \alpha)$ quando o modelo é especificado para a PROC GLM na ordem A, B, A*B.

Esta hipótese tem trés graus de liberdade e por isso, trés símbolos $\left.\mathrm{L} \mathrm{CL}_{5}, \mathrm{~L}_{6} \in \mathrm{L}_{7}\right)$ estão associados a ela.

Nota-se que $\mathrm{Ho}^{(5)}$ apesar de não conter os parámetros $\propto$ ainda é de dificil interpretaçăo, pois envolve funçбes pouco comuns dos parámetros $\boldsymbol{\gamma}$.

Em termos do Modelo M, Ho (S) fica:

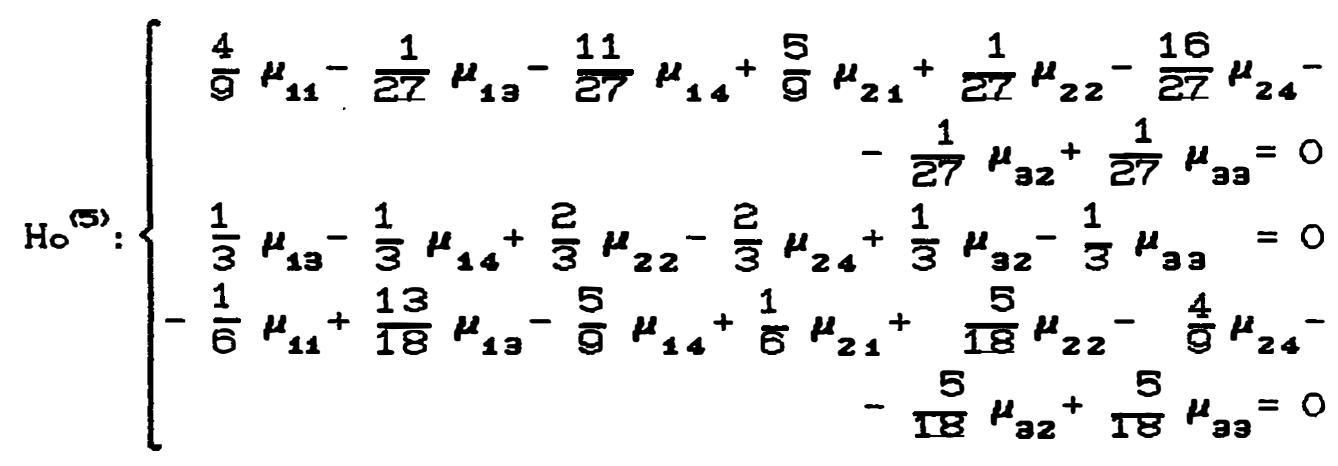

Para obter-se sua soma de quadrados,

calcula-se

$$
R(\beta \mid \mu, \alpha)=S Q H_{0}^{(5)}=\left[B^{\prime}(5)^{\prime} \mu^{0}\left[B_{(5)}^{\prime}\left(W^{\prime} W\right)^{-1} B_{(5)}\right]^{-1}\left[B^{\prime}(5)^{\prime} \mu^{0}\right]\right.
$$

onde: 


$$
B_{15)}^{\prime}=\left[\begin{array}{cccccccc}
4 / 8 & -1 / 27 & -11 / 27 & 5 / 9 & 1 / 27 & -16 / 27 & -1 / 27 & 1 / 27 \\
0 & 1 / 3 & -1 / 3 & 0 & 2 / 3 & -2 / 3 & 1 / 3 & -1 / 3 \\
-1 / 6 & 13 / 18 & -5 / 9 & 1 / 6 & 5 / 18 & -4 / 9 & -5 / 18 & 5 / 18
\end{array}\right]
$$

Para a interação entre espaçamentos e doses, as funçz̃es estimáveis tipo I são obtidas através de:

$$
\begin{aligned}
& G_{3}=C 0|0| X_{3}^{\prime} M_{2} X_{3}{ }^{3} \\
& \text { onde } \quad M_{2}=M_{1}-M_{1} X_{2}\left(X_{2}^{\prime} M_{1} X_{2}\right)^{-} X_{2}^{\prime} M_{1}=
\end{aligned}
$$

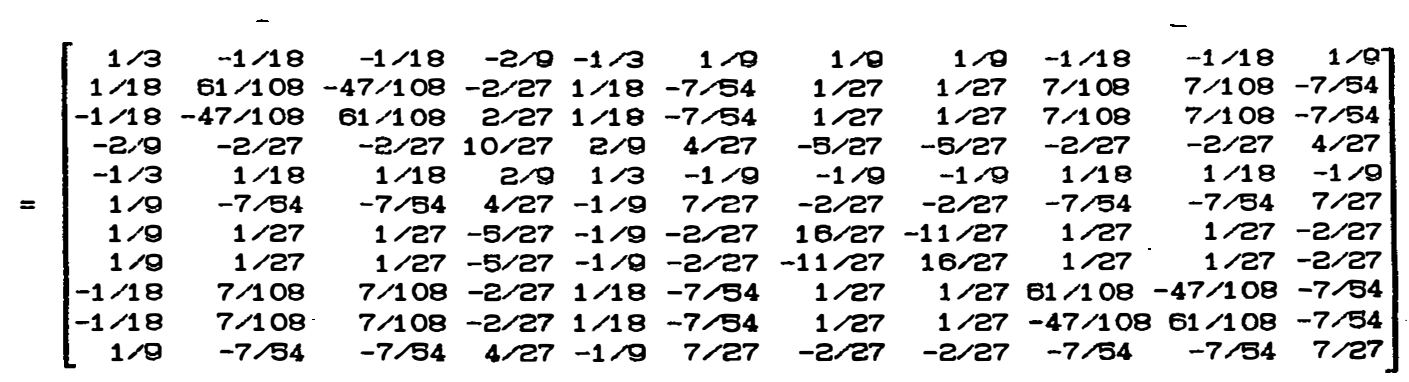

$$
\begin{aligned}
& G_{3}^{*}=\left(X_{3}^{\prime} M_{2} X_{3}\right)^{-} G_{3}= \\
& =\left[\begin{array}{rrrrrrrrrrrrrrrr}
0 & 0 & 0 & 0 & 0 & 0 & 0 & 0 & 1 & 0 & -1 & -1 & 0 & 1 & 0 & 0 \\
0 & 0 & 0 & 0 & 0 & 0 & 0 & 0 & 0 & 1 & -1 & 0 & -1 & 1 & 1 & -1 \\
0 & 0 & 0 & 0 & 0 & 0 & 0 & 0 & 0 & 0 & 0 & 0 & 0 & 0 & 0 & 0 \\
0 & 0 & 0 & 0 & 0 & 0 & 0 & 0 & 0 & 0 & 0 & 0 & 0 & 0 & 0 & 0 \\
0 & 0 & 0 & 0 & 0 & 0 & 0 & 0 & 0 & 0 & 0 & 0 & 0 & 0 & 0 & 0 \\
0 & 0 & 0 & 0 & 0 & 0 & 0 & 0 & 0 & 0 & 0 & 0 & 0 & 0 & 0 & 0 \\
0 & 0 & 0 & 0 & 0 & 0 & 0 & 0 & 0 & 0 & 0 & 0 & 0 & 0 & 0 & 0 \\
0 & 0 & 0 & 0 & 0 & 0 & 0 & 0 & 0 & 0 & 0 & 0 & 0 & 0 & 0 & 0
\end{array}\right]_{10}^{L_{1}}
\end{aligned}
$$$$
G_{3}^{*} \theta \text { fornece as funçßes estimáveis tipo I para }
$$

a interação AxB apresentadas na Tabela 28. 
Tabel a 28 - Funções Estimáveis Tipo I para AxB.

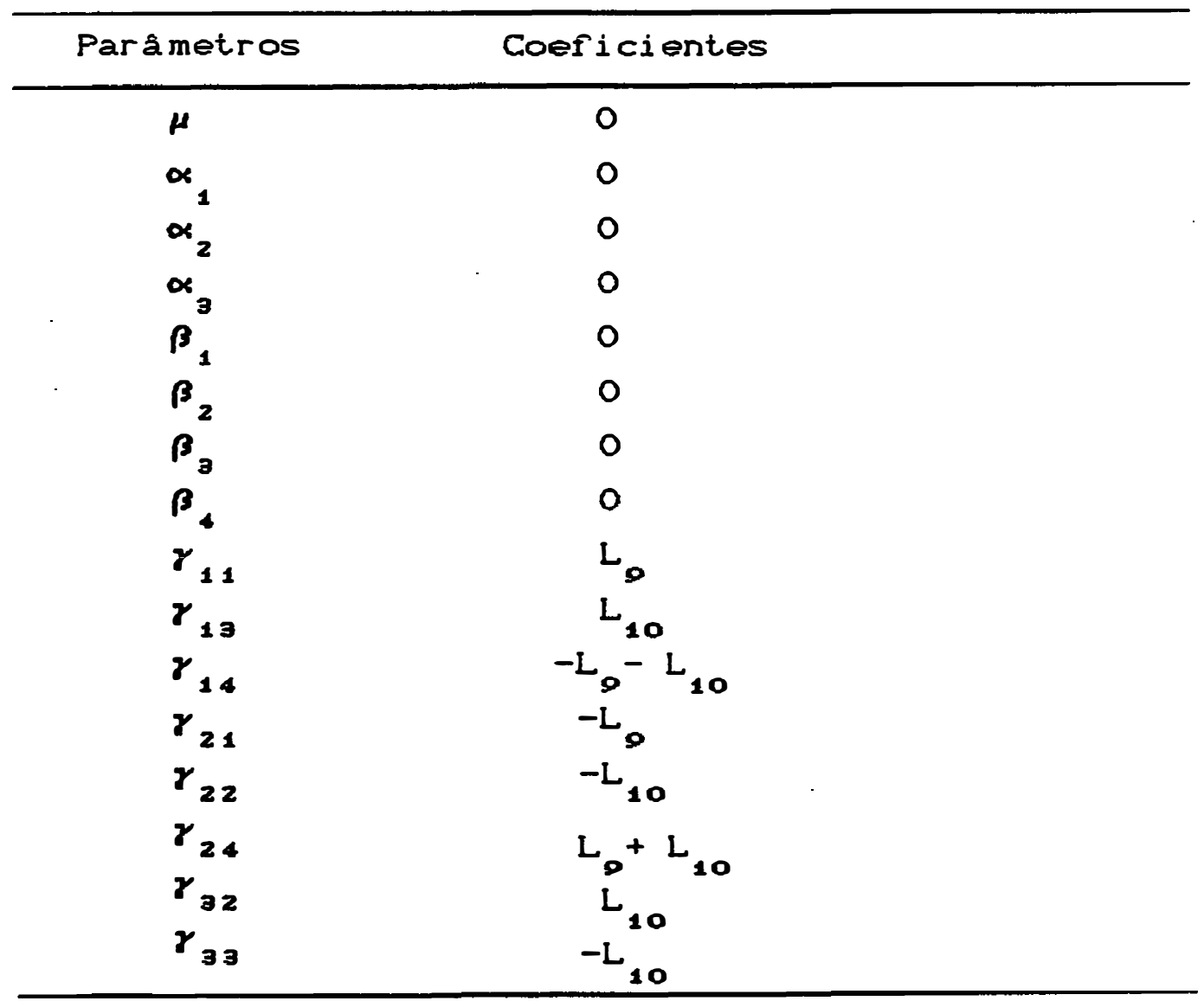

Como dois simbolos $\mathrm{L}_{\mathrm{CL}}$ e $\mathrm{L}_{10}$ ) eståo envolvidos, a hipótese associada tem dois graus de 1 iberdade.

Fazendo-se, digamos, $L_{\text {g }}$ igual a $1, L_{10}$ igual a zero; depois $L_{9}$ igual a zero e $L_{10}$ igual. a 1 , obtém-se:

$$
H 0^{(7)}: \begin{cases}\gamma_{11}-\gamma_{14}-\gamma_{21}+\gamma_{24} & =0 \\ \gamma_{13}-\gamma_{14}-\gamma_{22}+\gamma_{24}+\gamma_{32}-\gamma_{33}=0\end{cases}
$$

A hipótese sobre a interação é livre dos outros parámetros.

$$
\text { Caso não houvessem caselas vazias neste }
$$


125.

exemplo, haveriam $(a-1)(b-1)=6$ graus de liberdade para a interação que seria composta por seis funçơes lineares deltasc $\Delta$ linearmente independentes.

Neste caso, a interação tem somente $S-(a+b-1)=8-6=2$ graus de liberdade e portanto só duas funçơes linearmente independentes poderão ser testadas.

Observando-se o padrão dos dados, verifica-se que $\Delta_{11,24}$ estimável, pois todos os seus componentes correspondem a casela completas.

$$
\Delta_{11,24}=\mu_{11}-\mu_{14}-\mu_{21}+\mu_{24}
$$

Al ém deste, não se dispõe de outro $\Delta$ cujos elementos sejam todos estimáveis.

Mas uma soma ou diferença entre deltas c $\Delta$ pode ser estimável, pois existe outro grau de liberdade para interação.

Assim

$$
\begin{gathered}
\left.\Delta_{13,34}+\Delta_{24,32}=c \mu_{13}-\mu_{14}-\mu_{39}+\mu_{34}\right)+\left(\mu_{24}-\mu_{22}-\mu_{34}+\mu_{32}\right) \\
=\mu_{13}-\mu_{14}-\mu_{93}+\mu_{24}-\mu_{22}+\mu_{32}
\end{gathered}
$$

e então

$$
H_{0}^{(7)}:\left\{\begin{array}{l}
\Delta_{11,24}=0 \\
\Delta_{13,34}+\Delta_{24,32}=0
\end{array}\right.
$$

Em termos do Modelo $M$, a hipótese $\mathrm{Ho}^{(7)}$,

fica:

$$
H_{0}^{(7)}: \begin{cases}\mu_{11}-\mu_{14}-\mu_{21}+\mu_{24} & =0 \\ \mu_{13}-\mu_{14}-\mu_{22}+\mu_{24}+\mu_{32}-\mu_{93} & =0\end{cases}
$$


A soma de quadrados tipo I para interação AxB é obtida, calcul ando-se

$$
\begin{aligned}
& R(\gamma \mid \mu, \alpha, \beta)=S Q H_{0}^{(7)}=\left[B_{(7)^{\prime}}^{\prime}\right]^{\circ}\left[B_{(7)}^{\prime}\left(w^{\prime} w\right)^{-1} B_{(7)}\right]^{-1}\left[B_{(7)^{\prime}}^{\prime} \mu^{\circ}\right. \\
& \text { onde } \\
& B_{(7)}^{\prime}=\left[\begin{array}{rrrrrrrr}
1 & 0 & -1 & -1 & 0 & 1 & 0 & 0 \\
0 & 1 & -1 & 0 & -1 & 1 & 1 & -1
\end{array}\right] \\
& R(\gamma \mid \mu, \alpha, \beta)=S Q H_{0}^{(7)}=24,750
\end{aligned}
$$

\section{2. 4. Obtenção das Fumçōes Estimáveis Tipo II}

Para obtenção das funç̧̋es estimáveis tipo II para o fator A (espaçamentos) considera-se que o efeito do fator A está contido no efeito da interação AxB.

A construção se dá da seguinte forma:

- as colunas de $L$ associadas ao fator $B$ serão preenchidas por zeros;

- a submatriz associada com o fator $A$ sera $\left(X_{1} M_{1} X^{-}\left(X_{1}^{\prime} M X_{1}\right)\right.$;

- a submatriz associada com o fator AxB será

$$
\left(X_{1}: M X_{1}\right)^{-}\left(X_{1}: M_{2}\right)
$$

onde:

$X_{0} e$ formada pelas colunas da matriz $X$ correspondentes a $\mu e$ ao fator B.

$X_{1}$ é formada pelas colunas de $X$ correspondentes ao fator A.

$x_{2} \in$ formada pela colunas de $X$ correspondentes à interação AxB. 
127.

$$
\begin{aligned}
& X_{0}=\left[\begin{array}{lllll}
1 & 1 & 0 & 0 & 0 \\
1 & 0 & 0 & 1 & 0 \\
1 & 0 & 0 & 1 & 0 \\
1 & 0 & 0 & 0 & 1 \\
1 & 1 & 0 & 0 & 0 \\
1 & 0 & 1 & 0 & 0 \\
1 & 0 & 0 & 0 & 1 \\
1 & 0 & 0 & 0 & 1 \\
1 & 0 & 1 & 0 & 0 \\
1 & 0 & 1 & 0 & 0 \\
1 & 0 & 0 & 1 & 0
\end{array}\right] \quad X_{1}=\left[\begin{array}{lll}
1 & 0 & 0 \\
1 & 0 & 0 \\
1 & 0 & 0 \\
1 & 0 & 0 \\
0 & 1 & 0 \\
0 & 1 & 0 \\
0 & 1 & 0 \\
0 & 1 & 0 \\
0 & 0 & 1 \\
0 & 0 & 1 \\
0 & 0 & 1
\end{array}\right] \\
& x_{2}=\left[\begin{array}{llllllll}
1 & 0 & 0 & 0 & 0 & 0 & 0 & 0 \\
0 & 1 & 0 & 0 & 0 & 0 & 0 & 0 \\
0 & 1 & 0 & 0 & 0 & 0 & 0 & 0 \\
0 & 0 & 1 & 0 & 0 & 0 & 0 & 0 \\
0 & 0 & 0 & 1 & 0 & 0 & 0 & 0 \\
0 & 0 & 0 & 0 & 1 & 0 & 0 & 0 \\
0 & 0 & 0 & 0 & 0 & 1 & 0 & 0 \\
0 & 0 & 0 & 0 & 0 & 1 & 0 & 0 \\
0 & 0 & 0 & 0 & 0 & 0 & 1 & 0 \\
0 & 0 & 0 & 0 & 0 & 0 & 1 & 0 \\
0 & 0 & 0 & 0 & 0 & 0 & 0 & 1
\end{array}\right] \\
& M=I-x_{0}\left(X_{0} \cdot x_{0}\right)^{-} x_{0}^{1}= \\
& =\left[\begin{array}{ccccccccccc}
1 / 2 & 0 & 0 & 0 & -1 / 2 & 0 & 0 & 0 & 0 & 0 & 0 \\
0 & 2 / 3 & -1 / 3 & 0 & 0 & 0 & 0 & 0 & 0 & 0 & -1 / 3 \\
0 & -1 / 3 & 2 / 3 & 0 & 0 & 0 & 0 & 0 & 0 & 0 & -1 / 3 \\
0 & 0 & 0 & 2 / 3 & 0 & 0 & -1 / 3 & -1 / 3 & 0 & 0 & 0 \\
-1 / 2 & 0 & 0 & 0 & 1 / 2 & 0 & 0 & 0 & 0 & 0 & 0 \\
0 & 0 & 0 & 0 & 0 & 2 / 3 & 0 & 0 & -1 / 3 & -1 / 3 & 0 \\
0 & 0 & 0 & -1 / 3 & 0 & 0 & 2 / 3 & -1 / 3 & 0 & 0 & 0 \\
0 & 0 & 0 & -1 / 3 & 0 & 0 & -1 / 3 & 2 / 3 & 0 & 0 & 0 \\
0 & 0 & 0 & 0 & 0 & -1 / 3 & 0 & 0 & 2 / 3 & -1 / 3 & 0 \\
0 & 0 & 0 & 0 & 0 & -1 / 3 & 0 & 0 & -1 / 3 & 2 / 3 & 0 \\
0 & -1 / 3 & -1 / 3 & 0 & 0 & 0 & 0 & 0 & 0 & 0 & 2 / 3
\end{array}\right]
\end{aligned}
$$$$
L=\left[\left(x_{1}^{\prime} M x_{1}\right)^{-}\left(x_{1}^{\prime} M x_{1}\right) \phi \mid\left(x_{1}^{\prime} M x_{1}\right)^{-}\left(x_{1}^{\prime} M x_{2}\right)^{\prime}\right]=
$$ 
128.

$=\left[\begin{array}{cccc|cccc|cccccccc}0 & 1 & 0 & -1 & 0 & 0 & 0 & 0 & 1 / 6 & 11 / 18 & 2 / 9 & -1 / 6 & 7 / 18 & -2 / 8 & -7 / 18 & -11 / 18 \\ 0 & 0 & 1 & -1 & 0 & 0 & 0 & 0 & -1 / 6 & 7 / 18 & -2 / 9 & 1 / 8 & 11 / 18 & 2 / 9 & -11 / 18 & -7 / 18 \\ 0 & 0 & 0 & 0 & 0 & 0 & 0 & 0 & 0 & 0 & 0 & 0 & 0 & 0 & 0 & 0\end{array}\right] \mathrm{L}_{\mathrm{z}}$

Através de Le obtém-se as funçర̋es estimáveis tipo II para o fator A (espaçamentos), apresentadas na Tabela 29 .

Tabela 29 - FunçZ̃es Estimáveis Tipo II para A.

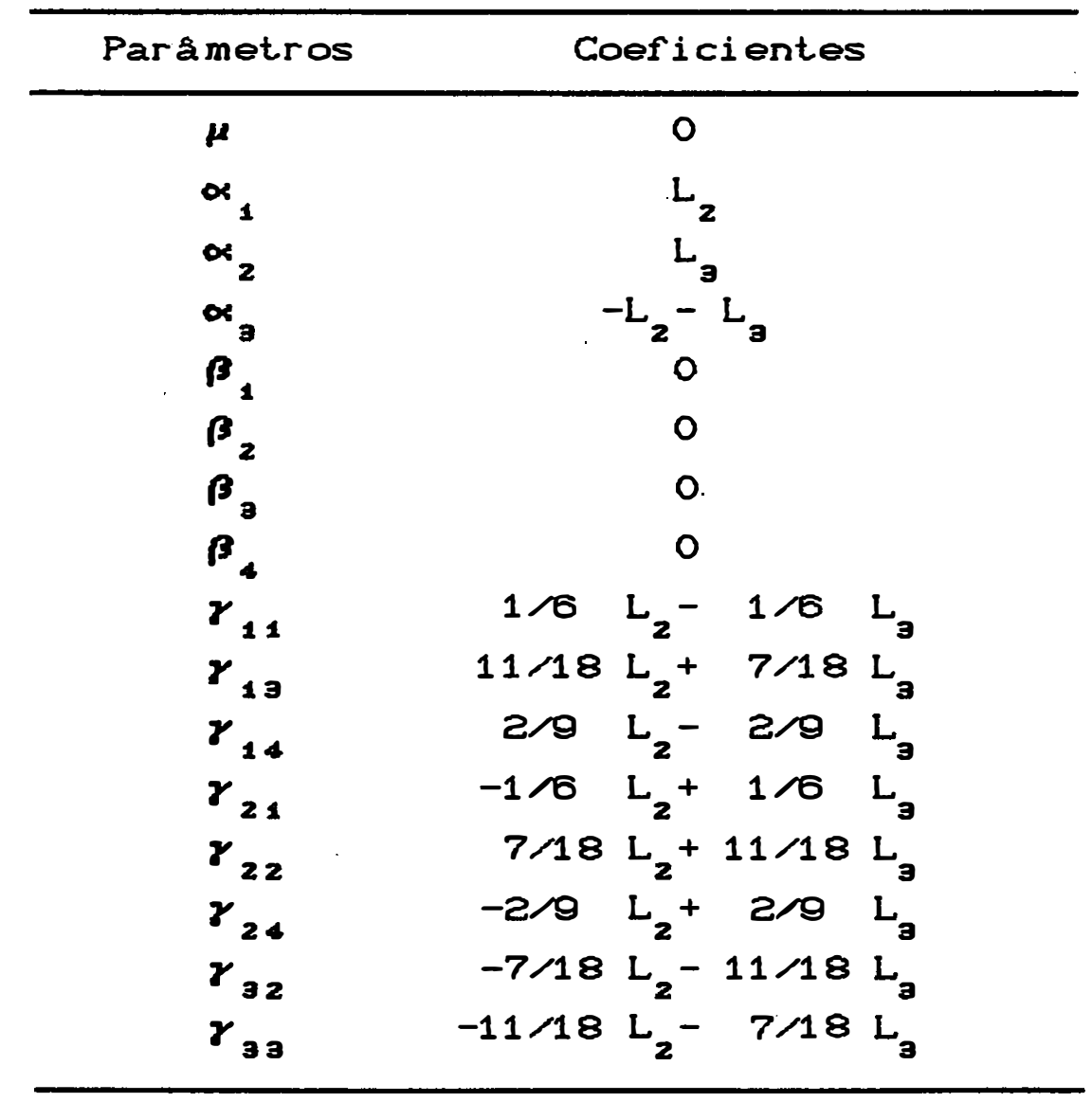

Para obter-se uma hipótese tipo II para o

fator $A$, faz-se primeiro, $L_{2}=1 \in L_{3}=0 ;$ depois, $L_{2}=0$ $\mathrm{L}_{3}=1$. 
129.

$$
H_{0}^{(2)}:\left\{\begin{array}{r}
\alpha_{1} \quad-\alpha_{3}+\frac{1}{6} \gamma_{11}+\frac{11}{18} \gamma_{13}+\frac{2}{\overline{9}} \gamma_{14}-\frac{1}{6} \gamma_{21}+\frac{7}{18} \gamma_{22} \\
-\frac{2}{9} \gamma_{24}-\frac{7}{18} \gamma_{32}-\frac{11}{18} \gamma_{33}=0 \\
\alpha_{2}-\alpha_{3}-\frac{1}{6} \gamma_{11}+\frac{7}{18} \gamma_{13}-\frac{2}{9} \gamma_{14}+\frac{1}{6} \gamma_{21}+\frac{11}{18} \gamma_{22} \\
+\frac{2}{9} \gamma_{24}-\frac{11}{18} \gamma_{32}-\frac{7}{18} \gamma_{33}=0
\end{array}\right.
$$

$H_{0}^{(2)}$ é a hipotese sobre médias ponderadas de linhas ajustadas para colunas. Em termos da notação RC J, a hipótese $H_{0}^{(2)} e$ dada por $\left.R C \alpha \mid \mu, \beta\right)$. Apesar de não envolver os parámetros $\beta$, a hipótese $e$ dificil de interpretar pois envolve combinaç̧es lineares dos parametros $\gamma$,bastante complexas.

No Modelo $\mathrm{M}, \mathrm{Ho}^{(2)}$ fica

$$
H o{ }^{(2)}:\left\{\begin{array}{r}
\frac{1}{6} \mu_{11}+\frac{11}{18} \mu_{13}+\frac{2}{9} \mu_{14}-\frac{1}{5} \mu_{21}+\frac{7}{18} \mu_{22} \\
-\frac{2}{9} \mu_{24}-\frac{7}{18} \mu_{32}-\frac{11}{18} \mu_{33}=0 \\
-\frac{1}{6} \mu_{11}+\frac{7}{18} \mu_{13}-\frac{2}{9} \mu_{14}+\frac{1}{\overline{6}} \mu_{21}+\frac{11}{18} \mu_{22} \\
+\frac{2}{9} \mu_{24}-\frac{11}{18} \mu_{32}-\frac{7}{18} \mu_{33}=0
\end{array}\right.
$$

A soma de quadrados tipo II para o fator A

dada por

$$
R C \alpha \mid \mu, \beta)=S Q H_{0}^{(2)}=\left[B_{(2)^{\prime}}^{\prime} \mu^{0}\right]^{\prime}\left[B_{(2)}^{\prime}\left(w^{\prime} w\right)^{-1} B_{(2)}\right]^{-1}\left[B^{\prime}(2)^{\prime} \mu^{0}\right]
$$

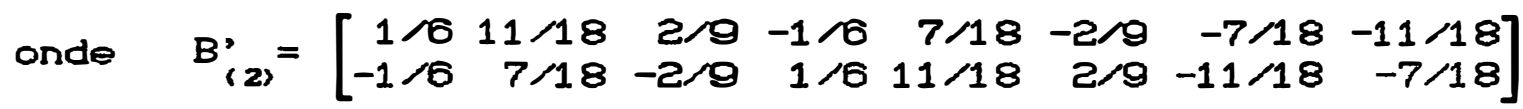

$$
R(\alpha \mid \mu, \beta)=S Q H_{0}^{(2)}=2238,083
$$

Para obtenção das funçås estimáveis tipo II 
para o fator B, considera-se que o efeito do fator B esta contido no efeito da interação $\mathrm{AxB} e$ não está contido no efeito do fator A.

$$
\mathrm{x}_{0}=\left[\begin{array}{llll}
\text { Então } & & & \\
1 & 1 & 0 & 0 \\
1 & 1 & 0 & 0 \\
1 & 1 & 0 & 0 \\
1 & 1 & 0 & 0 \\
1 & 0 & 1 & 0 \\
1 & 0 & 1 & 0 \\
1 & 0 & 1 & 0 \\
1 & 0 & 1 & 0 \\
1 & 0 & 0 & 1 \\
1 & 0 & 0 & 1 \\
1 & 0 & 0 & 1
\end{array}\right]
$$

onde

$X_{0}$ é formada pelas colunas de $X$ correspondentes à $\mu$ ao fator A;

$X_{1} \in$ formada pelas colunas de $X$ correspondentes ao fator $B$;

$X_{2}$ é formada pelas colunas de $X$ correspondentes à interação $A * B$.

Observa-se que para a construção das funçశ̋es estimáveis tipo II, procede-se de forma independente para cada fator. Isto não ocorre com as funçơes estimáveis tipo I pois seu ajuste é sequencial e sua obtenção para um 
131.

determinado fator depende do fator que o antecede.

- gerador das funçठes estimáveis tipo II para

- fator $B$ é dado por

$$
\begin{aligned}
& L=\left[\phi\left|\left(X_{1}^{\prime} M_{1}\right)^{-} X_{1}^{\prime} M X_{1}\right|\left(X_{1}^{\prime} M X_{1}\right)^{-} X_{1}^{\prime} M X_{2}\right] \\
& M_{1}=I-x_{1}\left(x_{1}: x_{1}\right)^{-} x_{1}^{\prime}= \\
& =\left[\begin{array}{ccccccccccc}
3 / 4 & -1 / 4 & -1 / 4 & -1 / 4 & 0 & 0 & 0 & 0 & 0 & 0 & 0 \\
-1 / 4 & 3 / 4 & -1 / 4 & -1 / 4 & 0 & 0 & 0 & 0 & 0 & 0 & 0 \\
-1 / 4 & -1 / 4 & 3 / 4 & -1 / 4 & 0 & 0 & 0 & 0 & 0 & 0 & 0 \\
-1 / 4 & -1 / 4 & -1 / 4 & 3 / 4 & 0 & 0 & 0 & 0 & 0 & 0 & 0 \\
0 & 0 & 0 & 0 & 3 / 4 & -1 / 4 & -1 / 4 & -1 / 4 & 0 & 0 & 0 \\
0 & 0 & 0 & 0 & -1 / 4 & 3 / 4 & -1 / 4 & -1 / 4 & 0 & 0 & 0 \\
0 & 0 & 0 & 0 & -1 / 4 & -1 / 4 & 3 / 4 & -1 / 4 & 0 & 0 & 0 \\
0 & 0 & 0 & 0 & -1 / 4 & -1 / 4 & -1 / 4 & 3 / 4 & 0 & 0 & 0 \\
0 & 0 & 0 & 0 & 0 & 0 & 0 & 0 & 2 / 3 & -1 / 3 & -1 / 3 \\
0 & 0 & 0 & 0 & 0 & 0 & 0 & 0 & -1 / 3 & 2 / 3 & -1 / 3 \\
0 & 0 & 0 & 0 & 0 & 0 & 0 & 0 & -1 / 3 & -1 / 3 & 2 / 3
\end{array}\right]
\end{aligned}
$$

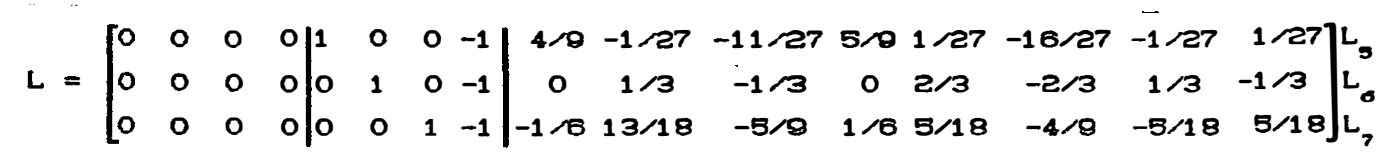

Lo dá as funçôes estimáveis tipo II para o fator B (doses), apresentadas na Tabela 30. 
132.

Tabela 30 - Funçơes Estimáveis Tipo II para B.

\begin{tabular}{lc}
\hline Parametros & Coeficientes \\
\hline$\alpha_{1}$ & 0 \\
$\alpha_{2}$ & 0 \\
$\alpha_{3}$ & 0 \\
$\beta_{1}$ & $\mathrm{~L}_{5}$ \\
$\beta_{2}$ & $\mathrm{~L}_{6}$ \\
$\beta_{3}$ & $\mathrm{~L}_{7}$ \\
$\beta_{4}$ & $-\mathrm{L}_{5}-\mathrm{L}_{6}-\mathrm{L}_{7}$ \\
$\gamma_{11}$ & $4 / 9 \mathrm{~L}_{5}-1 / 6 \mathrm{~L}_{7}$ \\
$\gamma_{13}$ & $-1 / 27 \mathrm{~L}_{5}+1 / 3 \mathrm{~L}_{6}+13 / 18 \mathrm{~L}_{7}$ \\
$\gamma_{14}$ & $-11 / 27 \mathrm{~L}_{5}-1 / 3 \mathrm{~L}_{6}-5 / 9 \mathrm{~L}_{7}$ \\
$\gamma_{21}$ & $5 / 9 \mathrm{~L}_{5}+1 / 6 \mathrm{~L}_{7}$ \\
$\gamma_{22}$ & $1 / 27 \mathrm{~L}_{5}+2 / 3 \mathrm{~L}_{6}+5 / 18 \mathrm{~L}_{7}$ \\
$\gamma_{24}$ & $-16 / 27 \mathrm{~L}_{5}-2 / 3 \mathrm{~L}_{6}-4 / 9 \mathrm{~L}_{7}$ \\
$\gamma_{32}$ & $-1 / 27 \mathrm{~L}_{5}+1 / 3 \mathrm{~L}_{6}-5 / 18 \mathrm{~L}_{7}$ \\
$\gamma_{33}$ & $1 / 27 \mathrm{~L}_{5}-1 / 3 \mathrm{~L}_{6}+5 / 18 \mathrm{~L}_{7}$ \\
\hline
\end{tabular}

Observa-se que a Tabela 30 é igual a Tabela 27 e como era esperado, as funçőes estimáveis tipos I e II para o fator B são iguais, devido ao ajuste sequencial das funçớes estimáveis tipo $I$; ambas equivalem a $R(\beta \mid \mu, \alpha)$.

Para obtenção das funçỡes estimáveis tipo II para a interação AxB, basta tomar-se a forma geral de funçชes estimáveis apresentada na Tabela 25 e fazer-se $L_{1}$, $L_{2}, L_{3}, L_{4}, L_{5} \oplus L_{6}$ iguais a zero. 
Tabela 31 - Funçठ̋es Estimáveis Tipo II para AxB

\begin{tabular}{cc}
\hline Parametros & Coeficientes \\
\hline$\alpha_{1}$ & 0 \\
$\alpha_{2}$ & 0 \\
$\alpha_{3}$ & 0 \\
$\beta_{1}$ & 0 \\
$\beta_{2}$ & 0 \\
$\beta_{3}$ & 0 \\
$\beta_{4}$ & 0 \\
$\gamma_{11}$ & 0 \\
$\gamma_{13}$ & $\mathrm{~L}_{0}$ \\
$\gamma_{14}$ & $\mathrm{~L}_{10}$ \\
$\gamma_{21}$ & $-\mathrm{L}_{0}^{-\mathrm{L}_{10}}$ \\
$\gamma_{22}$ & $-\mathrm{L}_{0}$ \\
$\gamma_{24}$ & $-\mathrm{L}_{10}$ \\
$\gamma_{32}$ & $\mathrm{~L}_{0}^{+\mathrm{L}_{10}}$ \\
$\gamma_{33}$ & $\mathrm{~L}_{10}$ \\
\hline
\end{tabular}

Que embora obtida de forma bem mais simples, e a mesma obtida através de $G_{3}^{*}{ }^{*}$ para a função estimavel tipo I.

Os quatro tipos de funçros estimáveis para a interação são iguais, pois as hipóteses para a interação aparecem sempre ajustadas para os demais efeitos.

4.2.5. Obtenção das Funçōes Estimáveis Tipo III

Para obter-se as funçбes estimáveis tipo II para o fator A: 
- tomar a forma geral de funçöes estimáveis e igualar $L_{5}$, $L_{6}$ e $\mathrm{L}_{7}$ a zero;

- obter $L_{9} e L_{10}$ en função de $L_{2} e L_{3}$, a fim de fazer a hipótese de A ortogonal à hipótese de AxB, da mesma forma apresentada para o fator B no exemplo anterior.

obtém-se $\mathrm{L}_{9}=1 / 5 \mathrm{~L}_{2}-1 / 5 \mathrm{~L}_{3}$

$$
L_{10}=3 / 5 L_{2}+2 / 5 L_{3}
$$

que dão origem as funçర̋es estimaveis tipo III para o fator A (espaçamentos), apresentadas na Tabela 32.

Tabela 32 - Funçỡes Estimáveis Tipo III para A.

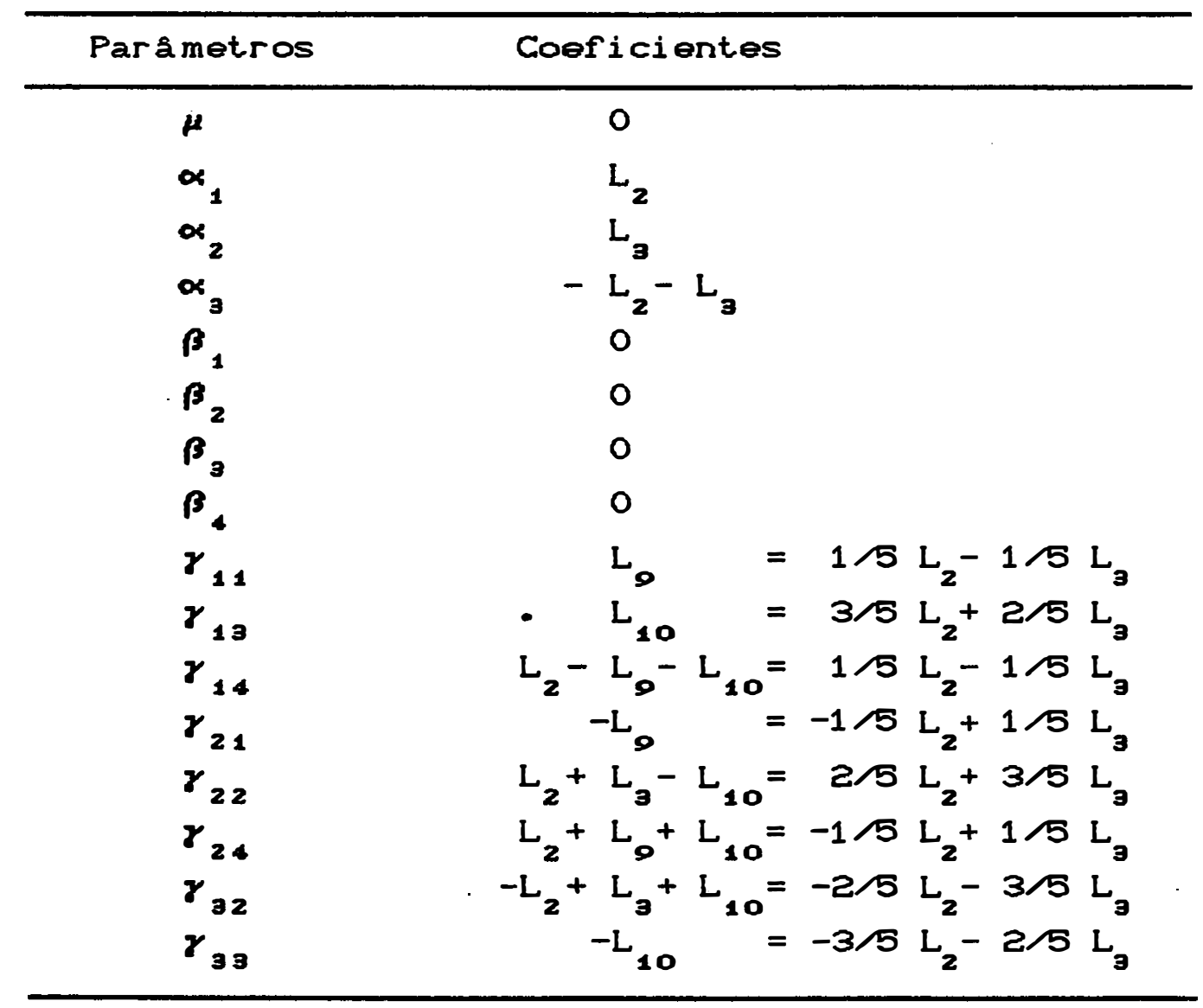


Somando-se os coeficientes de $\boldsymbol{\gamma}_{11}, \boldsymbol{\gamma}_{13}$ e $\boldsymbol{\gamma}_{14}$, obtém-se $L_{2}$ que $e$ o coeficiente de $\alpha_{1}$. Somando-se os coeficientes de $\gamma_{21}, \gamma_{22} e \gamma_{24}$, obtém-se $L_{3}$ que é 0 coeficiente de $\alpha_{2}$. Somando-se os coeficientes de $\boldsymbol{\gamma}_{32} e \boldsymbol{\gamma}_{33}$ obtém-se $-\mathrm{L}_{2}-\mathrm{L}_{3}$ que é o coeficiente de $\alpha_{3}$. A soma dos coeficientes de $\boldsymbol{\gamma}_{11} e \boldsymbol{\gamma}_{21}$ deve ser zero coeficiente de $\left.\beta_{1}\right)$, assim também para $\gamma_{13}$ mais $\gamma_{33}\left(\beta_{3}\right) e \gamma_{22}$ mais $\gamma_{32}$ $\left(\beta_{2}\right)$.

Concluindo-se que as hipóteses são ortogonais.

$$
\text { Fazendo-se } L_{2} \text { igual a } 1 \text { e } L_{3} \text { igual a zero, }
$$
para a primeira linha; $L_{2}$ igual a zero e $L_{3}$ igual a 1 para a segunda linha, obtém-se a hipotese $\mathrm{Ho}^{\text {(9) }}$.

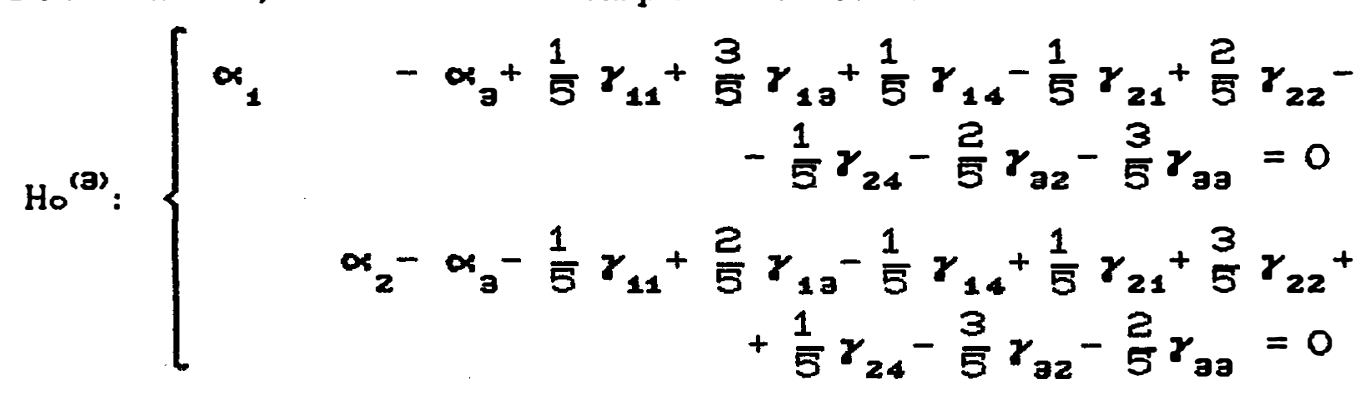

que é uma hipótese sobre médias não ponderadas de linhas.

As somas de quadrados tipo III se equivalem a $R(\dot{\alpha} \mid \dot{\mu}, \dot{\beta}, \dot{\gamma})$ em termos da notaça $R C 2$.

A hipotese $H_{0}^{(3)}$ em termos dos parámetros do Modelo $\boldsymbol{\Sigma}$ seria

$$
H_{0}^{(a)}: \quad \dot{\alpha}_{i}=0 \quad \forall i
$$

e a hipótese $H_{0}^{(3)}$ anterior é a hipótese tipo III para A escrita em termos dos parámetros do Modelo $S$. Dal a justificativa do aparecimento dos parámetros $\boldsymbol{\gamma}$. 
As funçros estimáveis tipo III para o fator A também podem ser obtidas pelo Método de SEARLE (1980), da seguinte forma.

$$
\text { - primeiro passo é construir-se }
$$
contrastesbaseados nos parâmetros $\alpha$, que não envol vam os $\beta$ e que considerem somente as caselas que possuem dados.

Contrastes

$\alpha_{1}-\alpha_{2}+1 / 2\left(\gamma_{11}+\gamma_{14}\right)-1 / 2\left(\gamma_{21}+\gamma_{24}\right)$

$\alpha_{1}-\alpha_{3}+\gamma_{13}-\gamma_{33}$

$\alpha_{2}-\alpha_{3}+\gamma_{22}-\gamma_{13}$

Usando coeficientes $m_{1}, m_{2}$ e $m_{3}$ Cum para cada linha do contraste), constrói-se uma funçao linear

$$
\begin{aligned}
& f=m_{1}\left[\alpha_{1}-\alpha_{2}+1 / 2\left(\gamma_{11}+\gamma_{14}\right]-1 / 2\left(\gamma_{21}+\gamma_{24}\right)\right]+ \\
&+m_{2}\left[\alpha_{1}-\alpha_{a}+\gamma_{1 a}-\gamma_{a 3}\right]+m_{a}\left[\alpha_{2}-\alpha_{a}+\gamma_{22}-\gamma_{a 2}\right] \\
& 0 \text { contraste para compor } R(\gamma \mid \mu, \alpha, \beta) j a \text { foi }
\end{aligned}
$$

visto ser:

$$
H_{0}^{(7)}: \begin{cases}\gamma_{11}-\gamma_{14}-\gamma_{21}+\gamma_{24} & =0 \\ \gamma_{13}-\gamma_{14}-\gamma_{22}+\gamma_{24}+\gamma_{32}-\gamma_{33}=0\end{cases}
$$

Fazendo-se os contrastes de $\alpha$ ortogonais a estes, obtém-se: 
Tabela 33 - Coeficientes para a Combinação Linear $f$ e para a Interação

\begin{tabular}{|c|c|c|c|c|c|c|c|}
\hline \multirow[b]{3}{*}{$\begin{array}{l}\text { contraste } \\
\text { conts }\end{array}$} & \multirow[t]{3}{*}{$\alpha$} & \multicolumn{6}{|c|}{$\gamma$} \\
\hline & & $\alpha_{1}$ & $\alpha_{2}$ & $\alpha_{3}$ & $\gamma_{11}$ & $\gamma_{13}$ & $\gamma_{14}$ \\
\hline & & $\begin{array}{c}m_{1}+m_{2} \\
0\end{array}$ & $\begin{array}{c}-m_{1}+m_{3} \\
0\end{array}$ & $\begin{array}{c}-m_{2}-m_{3} \\
0\end{array}$ & $\begin{array}{c}1 / 2 m_{1} \\
1\end{array}$ & $\begin{array}{c}m_{2} \\
1\end{array}$ & $\begin{array}{c}1 / 2 m_{1} \\
-2\end{array}$ \\
\hline & & & & $\gamma$ & & & \\
\hline & & $\gamma_{21}$ & $\gamma_{22}$ & $\gamma_{24}$ & $\gamma_{32}$ & & $\mathbf{3} \mathbf{3}$ \\
\hline $\begin{array}{l}\text { contraste } \\
\text { contrate }\end{array}$ & $\gamma$ & $\begin{array}{c}-1 / 2 m_{1} \\
-1\end{array}$ & $\begin{array}{l}m_{3} \\
-1\end{array}$ & $\begin{array}{c}-1 / 2 m_{1} \\
2\end{array}$ & $\begin{array}{c}-m_{3} \\
1\end{array}$ & - & $\begin{array}{l}m_{2} \\
-1\end{array}$ \\
\hline
\end{tabular}

A ortogonalidade entre as duas hipóteses i mplica:

$$
\begin{gathered}
1 / 2 m_{1}+m_{2}-m_{1}+1 / 2 m_{1}-m_{3}-m_{1}-m_{3}+m_{2}=0 \\
m_{1}=2 m_{2}-2 m_{3}
\end{gathered}
$$

A hipótese para o fator A tem dois graus de liberdade, então existem dois valores arbitrários para os m, $L_{2} \in L_{3}$

$$
\text { Sendo } \mathrm{L}_{2} \alpha_{1}+\mathrm{L}_{3} \alpha_{2} \text {, }
$$

$$
\text { entao } L_{2}=m_{1}+m_{2} \text { e } L_{3}=-m_{1}+m_{3}
$$

obtendo-se

$$
\begin{aligned}
& m_{1}=2 / 5 \mathrm{~L}_{2}-2 / 5 \mathrm{~L}_{3} \\
& m_{2}=3 / 5 \mathrm{~L}_{2}+2 / 5 \mathrm{~L}_{3} \\
& m_{3}=2 / 5 \mathrm{~L}_{2}+3 / 5 \mathrm{~L}_{3}
\end{aligned}
$$


138.

Substituindo em $f$, obtém-se:

$$
\begin{aligned}
f= & \mathrm{L}_{2} \alpha_{1}+\mathrm{L}_{3} \alpha_{2}+\left[-\mathrm{L}_{2}-\mathrm{L}_{3}\right] \alpha_{3}+\left[1 / 5 \mathrm{~L}_{2}-1 / 5 \mathrm{~L}_{3}\right] \gamma_{11}+ \\
& +\left[3 / 5 \mathrm{~L}_{2}+2 / 5 \mathrm{~L}_{3}\right] \gamma_{13}+\left[1 / 5 \mathrm{~L}_{2}-1 / 5 \mathrm{~L}_{3}\right] \gamma_{14}+\left[-1 / 5 \mathrm{~L}_{2}+1 / 5 \mathrm{~L}_{3}\right] \gamma_{21}+ \\
& +\left[2 / 5 \mathrm{~L}_{2}+3 / 5 \mathrm{~L}_{3}\right] \gamma_{22}+\left[-1 / 5 \mathrm{~L}_{2}+1 / 5 \mathrm{~L}_{3}\right] \gamma_{24}+\left[-2 / 5 \mathrm{~L}_{2}-3 / 5 \mathrm{~L}_{3}\right] \gamma_{32}+ \\
& +\left[-3 / 5 \mathrm{~L}_{2}-2 / 5 \mathrm{~L}_{3}\right] \gamma_{33}
\end{aligned}
$$

que são as funçชes estimáveis apresentadas na Tabela 27.

A hipótese Ho ${ }^{(a)}$ em termos dos parámetros do Modelo M fica:

$$
H_{0}^{(3)}:\left\{\begin{array}{c}
\frac{\mu_{11}+3 \mu_{13}+\mu_{14}-\mu_{21}+2 \mu_{22}-\mu_{24}-2 \mu_{32}-3 \mu_{33}}{5}=0 \\
\frac{\mu_{11}+2 \mu_{13}-\mu_{14}+\mu_{21}+3 \mu_{22}+\mu_{24}-3 \mu_{32}-2 \mu_{33}}{5}=0 \\
\text { Observa-se que a primeira lisha da hipotese }
\end{array}\right.
$$

faz a comparação entre linhas um e trés $\left(\alpha_{1} e \alpha_{3}\right)$. Porém, ambas envolvem uma comparação das médias da linha excluída entre si e apresentam coeficientes, tornando-a de dificil interpretação.

$$
\text { A soma de quadrados tipo III para o fator A, }
$$

é obtida calculando-se

$$
\begin{aligned}
& R(\dot{\alpha} \mid \dot{\mu}, \dot{\beta}, \dot{\gamma})=S Q H 0^{(3)}=\left[\mathrm{B}_{(3)}^{\prime} \mu^{\circ}\right]^{\prime}\left[\mathrm{B}_{(3)}^{\prime}\left(w^{\prime} w^{-1} \mathrm{~B}_{(3)}\right]^{-1}\left[\mathrm{~B}_{(3)^{\prime}} \mu^{0}\right]\right. \\
& \text { onde } \quad B^{\prime}(3)=\left[\begin{array}{rrrrrrrr}
1 / 5 & 3 / 5 & 1 / 5 & -1 / 5 & 2 / 5 & -1 / 5 & -2 / 5 & -3 / 5 \\
-1 / 5 & 2 / 5 & -1 / 5 & 1 / 5 & 3 / 5 & 1 / 5 & -3 / 5 & -2 / 5
\end{array}\right] \\
& R(\dot{\alpha} \mid \dot{\mu}, \dot{\beta}, \dot{\gamma})=\text { SQHo }^{(3)}=2212,804
\end{aligned}
$$


Observe que neste caso, $\mathrm{Ho}^{(3)}$ e bem diferente de $\mathrm{Ho}^{(2)}$.

Para obter-se as funçôs estimáveis tipo III

para o fator B:

- tomar a forma geral de funçőes estimáveis, apresentada na Tabela 25 e igual ar $\mathrm{L}_{1}, \mathrm{~L}_{2}$ e $\mathrm{L}_{3}$ a zero;

- obter $L_{9} e L_{10}$ em função de $L_{5}, L_{6} e L_{7}$, de modo a tornar a hipótese de $B$ ortogonal à hipótese de AxB, da mesma forma apresentada no exemplo anterior para o fator B, obtém-se

$\mathrm{L}_{9}=1 / 2 \mathrm{~L}_{5}+1 / 10 \mathrm{~L}_{6}-1 / 10 \mathrm{~L}_{7}$

$\mathrm{L}_{10}=3 / 10 \mathrm{~L}_{6}+7 / 10 \mathrm{~L}_{7}$.

As funçores estimáveis tipo II para o fator B obtidas por este processo estão apresentadas na Tabela 34. 
Tabela 34 - Funçס̋es Esti máveis Tipo III para B.

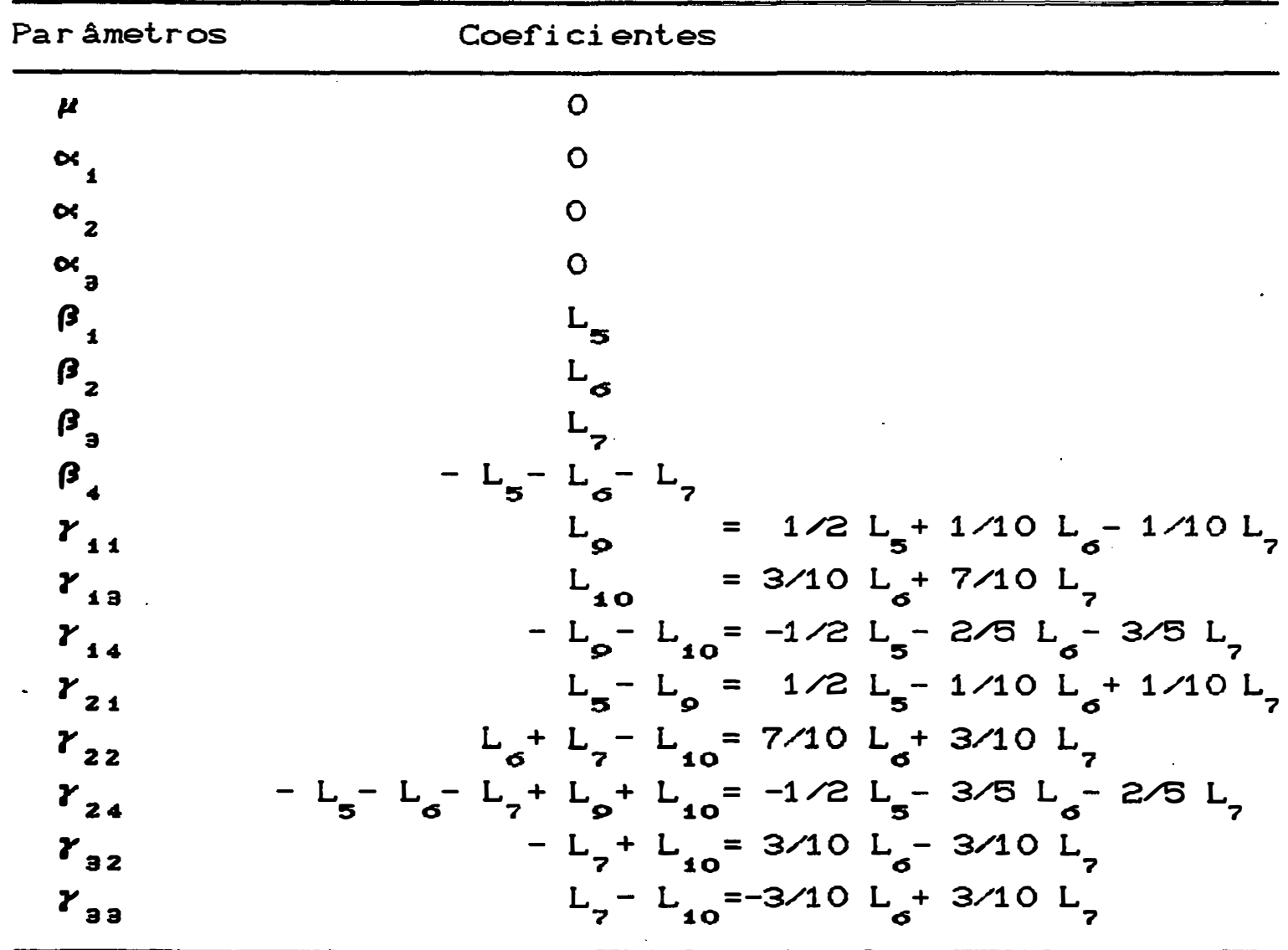

Somando-se as coeficientes de $\gamma_{11} e \gamma_{21}$, obtém-se $L_{5}$ que é o coeficiente de $\beta_{1}$.

Somando-se os coeficientes de $\boldsymbol{\gamma}_{22}$ e $\boldsymbol{\gamma}_{32}$, obtém-se $L_{6}$ que é o coeficiente de $\beta_{2}$.

Somando-se os coeficientes de $\gamma_{13} e \gamma_{33}$ ' obtém-se $L_{7}$ (coeficiente de $\beta_{3}$.

Somando-se os coeficientes de $\boldsymbol{\gamma}_{14}$ e $\boldsymbol{\gamma}_{24}$, obtém-se. $-\mathrm{L}_{5}-\mathrm{L}_{6}-\mathrm{L}_{7}$ coericiente de $\beta_{4}$.

Da mesma forma as somas dos coeficientes de $\boldsymbol{\gamma}_{11}, \boldsymbol{\gamma}_{13}, \boldsymbol{\gamma}_{14} ; \boldsymbol{\gamma}_{21}, \boldsymbol{\gamma}_{22}, \boldsymbol{\gamma}_{24} \propto \boldsymbol{\gamma}_{32}, \boldsymbol{\gamma}_{33}$ devem ser zero (coeficientes dos parámetros $\infty$. 
Uma hipótese tipo III para o fator B (doses), pode ser obtida, considerando-se : $L_{5}=1, L_{6}=0$ e $L_{7}=0$; $L_{5}=0, L_{6}=1 \in L_{7}=0 ; L_{5}=0, L_{6}=0 e L_{7}=1$

$$
H 0^{(0)}:\left\{\begin{aligned}
& \beta_{1}-\beta_{4}+\frac{1}{2} \gamma_{11}-\frac{1}{2} \gamma_{14}+\frac{1}{2} \gamma_{21}-\frac{1}{2} \gamma_{24}=0 \\
& \beta_{2}-\beta_{4}+\frac{1}{10} \gamma_{11}+\frac{3}{10} \gamma_{13}-\frac{2}{5} \gamma_{14}-\frac{1}{10} \gamma_{21}+ \\
&+\frac{7}{10} \gamma_{22}-\frac{3}{5} \gamma_{24}+\frac{3}{10} \gamma_{32}-\frac{3}{10} \gamma_{33}=0 \\
& \beta_{3}-\beta_{4}-\frac{1}{10} \gamma_{11}+\frac{7}{10} \gamma_{13}-\frac{3}{5} \gamma_{14}+\frac{1}{10} \gamma_{21}+ \\
&+\frac{3}{10} \gamma_{22}-\frac{2}{5} \gamma_{24}-\frac{3}{10} \gamma_{32}+\frac{3}{10} \gamma_{33}=0
\end{aligned}\right.
$$

que é uma hipótese sobre medias não ponderadas de col unas. Estes contrastes compre $R(\dot{\beta} \mid \dot{\mu}, \dot{\alpha}, \dot{\gamma}) . H_{0}^{(0)}$ em termos dos parámetros do Modelo $\boldsymbol{\Sigma}$ se equivale a

$$
H_{0}^{(0)}: \quad \dot{\beta}_{j}=0 \quad \forall j
$$

e assim se justifica a presença dos parámetros $\gamma$ quando a escrevemos em termos dos parámetros do Modelo S.

A hipótese $\mathrm{Ho}^{(0)}$ em termos dos parámetros do.

Modelo M fica:

$$
H 0^{(0)}: \quad\left\{\begin{array}{l}
\frac{\mu_{11}-\mu_{14}+\mu_{21}-\mu_{24}}{2}=0 \\
\frac{\mu_{11}+3 \mu_{13}-4 \mu_{14}-\mu_{21}+7 \mu_{22}-6 \mu_{24}+3 \mu_{32}-3 \mu_{33}}{10}=0 \\
\frac{-\mu_{11}+7 \mu_{13}-6 \mu_{14}+\mu_{21}+3 \mu_{22}-4 \mu_{24}-3 \mu_{32}+3 \mu_{33}}{10}=0
\end{array}\right.
$$


A primeira linha desta hipótese compara as colunas um e quatro, em suas casela completas. As outras duas linhas não trazem comparaçơes nitidas, além de coeficientes bastante confusos.

A soma de quadrados tipo III para o fator B

(doses), é dada por

$$
R(\dot{\beta} \mid \dot{\mu}, \dot{\alpha}, \dot{\gamma})=S Q H 0^{(\sigma)}=\left[B^{\prime}(\sigma)^{\mu^{0}}\right]^{\prime}\left[B^{\prime}(\sigma)^{\prime}\left(w^{\prime} w\right)^{-1} B_{(\sigma)}\right]^{-1}\left[B^{\prime}(\sigma)^{\prime}\right]
$$

onde $B^{\prime}(\sigma)=\left[\begin{array}{cccccccc}1 / 2 & 0 & -1 / 2 & 1 / 2 & 0 & -1 / 2 & 0 & 0 \\ 1 / 10 & 3 / 10 & -4 / 10 & -1 / 10 & 7 / 10 & -6 / 10 & 3 / 10 & -3 / 10 \\ -1 / 10 & 7 / 10 & -6 / 10 & 1 / 10 & 3 / 10 & -4 / 10 & -3 / 10 & 3 / 10\end{array}\right]$

$$
R(\dot{\beta} \mid \dot{\mu}, \dot{\alpha}, \dot{\gamma})=S Q H 0^{(O)}=1738,361
$$

A aplicação do algoritmo de SEARLE (1987) para converter as hipóteses em termos do Modelo $\Sigma$, $H_{0}^{(3)}: \dot{\alpha}_{i}=0 \quad \forall$ i e $H_{0}^{(0)}: \dot{\beta}_{j}=0 \quad \forall j$ em hipoteses em termos dos parámetros do Modelo $S$, será feita a seguir.

$$
\begin{aligned}
& W_{\mathbf{s}}=\left[\begin{array}{rrrrrrrr}
1 & 1 & 0 & 1 & 0 & 0 & 1 & 0 \\
1 & 1 & 0 & 0 & 0 & 1 & 0 & 1 \\
1 & 1 & 0 & -1 & -1 & -1 & -1 & -1 \\
1 & 0 & 1 & 1 & 0 & 0 & -1 & 0 \\
1 & 0 & 1 & 0 & 1 & 0 & 0 & -1 \\
1 & 0 & 1 & -1 & -1 & -1 & 1 & 1 \\
1 & -1 & -1 & 0 & 1 & 0 & 0 & 1 \\
1 & -1 & -1 & 0 & 0 & 1 & 0 & -1
\end{array}\right] \\
& W=\left[\begin{array}{llllllllllllllll}
1 & 1 & 0 & 0 & 1 & 0 & 0 & 0 & 1 & 0 & 0 & 0 & 0 & 0 & 0 & 0 \\
1 & 1 & 0 & 0 & 0 & 0 & 1 & 0 & 0 & 1 & 0 & 0 & 0 & 0 & 0 & 0 \\
1 & 1 & 0 & 0 & 0 & 0 & 0 & 1 & 0 & 0 & 1 & 0 & 0 & 0 & 0 & 0 \\
1 & 0 & 1 & 0 & 1 & 0 & 0 & 0 & 0 & 0 & 0 & 1 & 0 & 0 & 0 & 0 \\
1 & 0 & 1 & 0 & 0 & 1 & 0 & 0 & 0 & 0 & 0 & 0 & 1 & 0 & 0 & 0 \\
1 & 0 & 1 & 0 & 0 & 0 & 0 & 1 & 0 & 0 & 0 & 0 & 0 & 1 & 0 & 0 \\
1 & 0 & 0 & 1 & 0 & 1 & 0 & 0 & 0 & 0 & 0 & 0 & 0 & 0 & 1 & 0 \\
1 & 0 & 0 & 1 & 0 & 0 & 1 & 0 & 0 & 0 & 0 & 0 & 0 & 0 & 0 & 1
\end{array}\right]
\end{aligned}
$$


143.

$$
\beta_{s}=W^{-1} W
$$

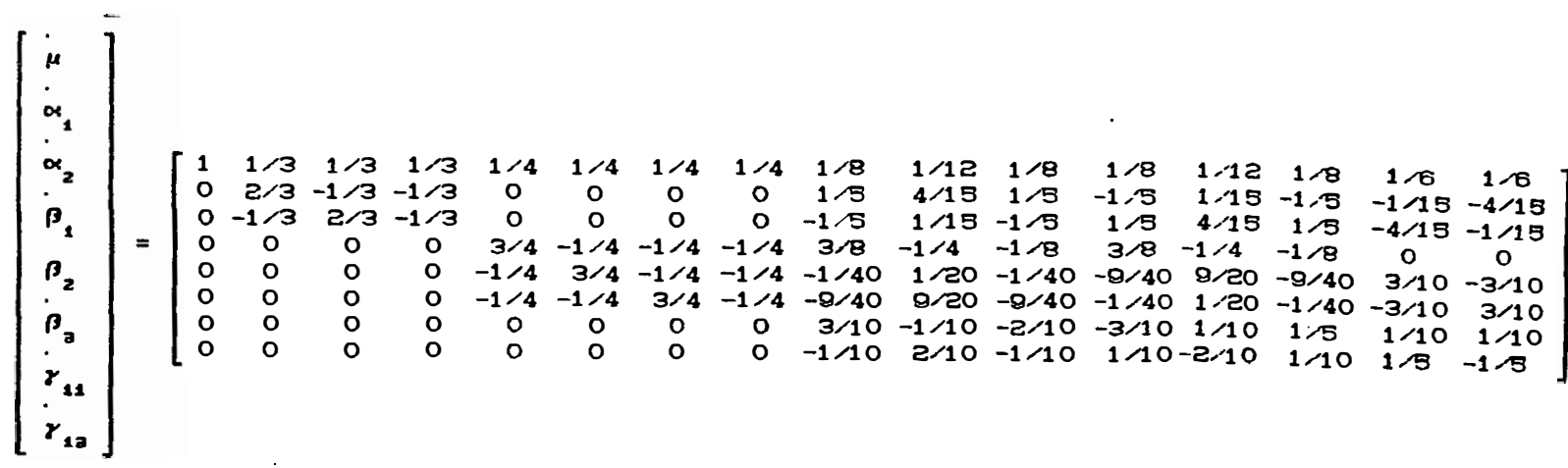

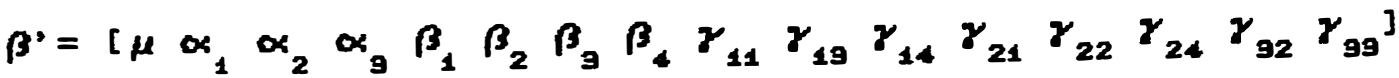

Assim

$$
\begin{aligned}
& \dot{\alpha}_{1}=\frac{2}{3} \alpha_{1}-\frac{1}{3} \alpha_{2}-\frac{1}{3} \alpha_{9}+\frac{1}{5} \gamma_{11}+\frac{4}{15} \gamma_{13}+\frac{1}{5} \gamma_{14}-\frac{1}{5} \gamma_{21}+\frac{1}{15} \gamma_{22} \\
& \dot{\alpha}_{2}=-\frac{1}{3} \alpha_{1}+\frac{2}{3} \alpha_{2}-\frac{1}{3} \alpha_{9}-\frac{1}{5} \gamma_{11}+\frac{1}{15} \gamma_{19}-\frac{1}{5} \gamma_{14}+\frac{1}{5} \gamma_{21}+\frac{4}{15} \gamma_{22}+\frac{4}{15} \gamma_{99} \\
& \dot{\beta}_{1}=\frac{3}{4} \beta_{1}-\frac{1}{4} \beta_{2}-\frac{1}{4} \beta_{9}-\frac{1}{4} \beta_{4}+\frac{3}{8} \gamma_{11}-\frac{1}{4} \gamma_{19}-\frac{1}{8} \gamma_{14}+\frac{3}{8} \gamma_{21} \\
& \dot{\beta}_{2}=-\frac{1}{4} \beta_{1}+\frac{3}{4} \beta_{2}-\frac{1}{4} \beta_{9}-\frac{1}{4} \beta_{49}-\frac{1}{40} \gamma_{11}+\frac{1}{20} \gamma_{19}-\frac{1}{40} \gamma_{14} \\
& \beta_{9}=-\frac{1}{4} \beta_{1}-\frac{1}{4} \beta_{2}+\frac{3}{4} \beta_{9}-\frac{1}{4} \beta_{4}-\frac{9}{40} \gamma_{11}+\frac{9}{20} \gamma_{19}-\frac{9}{40} \gamma_{14}- \\
& -\frac{9}{40} \gamma_{21}+\frac{1}{20} \gamma_{22}-\frac{1}{40} \gamma_{24}-\frac{3}{10} \gamma_{92}+\frac{3}{10} \gamma_{99}
\end{aligned}
$$

Convertendo-se as hipoteses $\mathrm{Ho}^{(9)}$ e $\mathrm{Ho}^{(0)} \mathrm{em}$ 
termos dos parametros do Modelo $\mathrm{S}$.

Observa-se que se nas funçன̋es estimaveis tipo

III para $A$, tormar-se primeiro $L_{2}=2 / 3$ e $L_{9}=-1 / 3$ e depois

$L_{2}=-1 / 3 e L_{3}=2 / 3$, obtem-se a hipótese $H_{0}^{(9)}:\left\{\begin{array}{l}\alpha_{1}=0 \\ \alpha_{2}=0\end{array}\right.$

escrita em função dos parămetros $\alpha \in \gamma$.

Se nas funçơes estimáveis tipo III para B,

considerar-se

$\mathrm{L}_{5}=3 / 4, \mathrm{~L}_{6}=-1 / 4$ \& $\mathrm{L}_{7}=-1 / 4$;

$L_{5}=-1 / 4, L_{6}=3 / 4$ \& $L_{7}=-1 / 4 ;$

$L_{5}=-1 / 4, L_{6}=-1 / 4 \in L_{7}=3 / 4$.

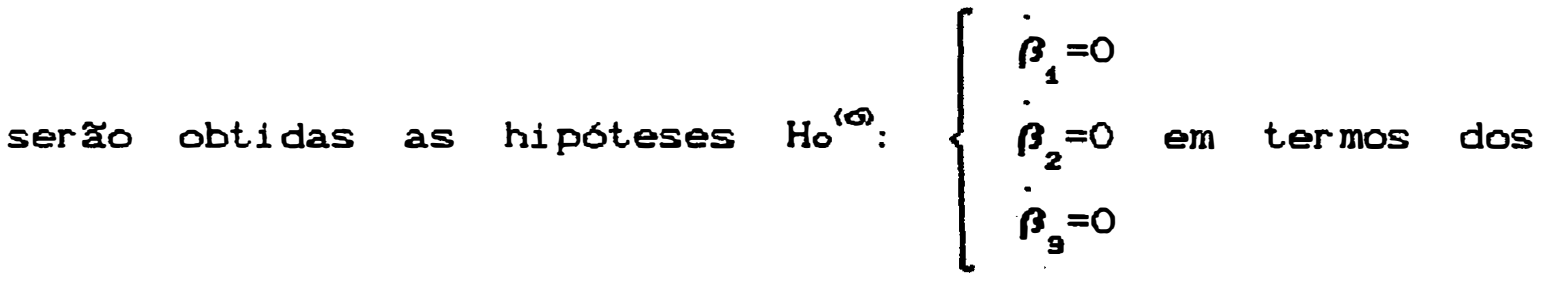
parámetros $\beta \in \gamma$.

\section{2.6. Obtenção das Funçōes Estimáveis Tipo IV}

Para obter-se as funçбes estimáveis tipo IV para o fator A (espaçamentos), se ira tomar a forma geral de funçães estimáveis apresentada na Tabela 25 e igualar $L_{1}$. $L_{5}, L_{6} \in L_{7}$ a zero.

Como a hipótese tipo IV para linhas só envolve as médias de colunas que possuem dados na liltima linha, $\mu_{11}, \mu_{21}, \mu_{14} \in \mu_{24}$ nå aparecerăo na hipótese tipo IV e por isso os coeficientes para $\gamma_{11}, \gamma_{14}, \gamma_{21}$ e $\gamma_{24}$ serão 
zeros.

Para escrever $L_{\phi} e L_{10}$ em função de $L_{2}$ Coeficiente de $\alpha_{1}$, observa-se que $L_{0}$ e o coeficiente de $\gamma_{11}$ e por isso já foi dito que deverá ser igual a zero. $L_{10}$ - o coeficiente de $\gamma_{19}$ ' o único componente da interaçăo correspondente a $\alpha_{1}$ que não difere de zero.

$$
\text { Assim, } \mathrm{L}_{10}=1 / \mathrm{KL}_{2} \text { e } \mathrm{K}=1 \text {. }
$$

As funçбes estimáveis resultantes deste processo são apresentadas na Tabela 35.

Tabela 35 - Funçס̋es Estimáveis Tipo IV para A.

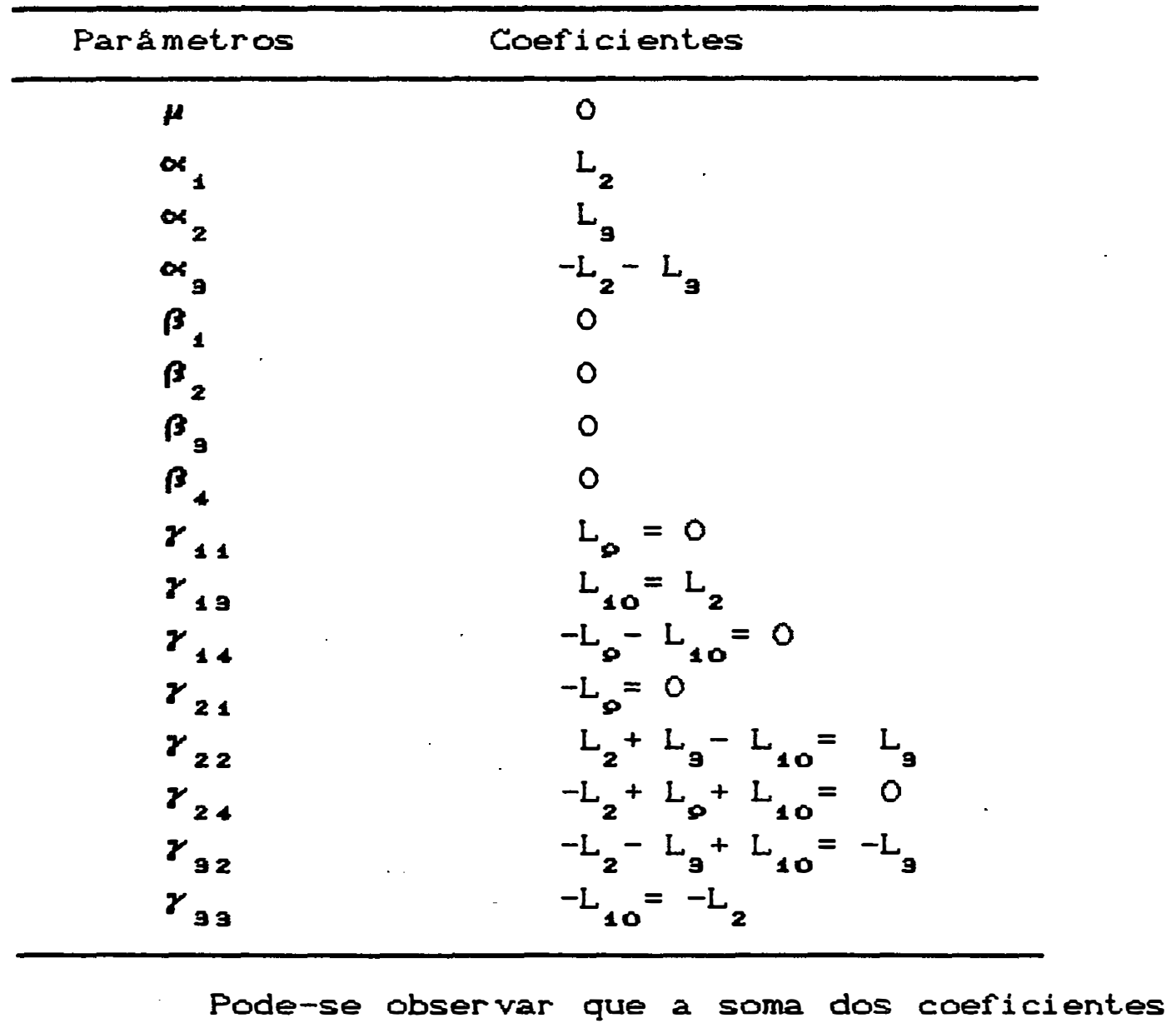


de $\gamma_{11}, \gamma_{19} e \gamma_{14} e$ igual a $L_{2}$ que $e 0$ coeficiente de $\alpha_{1}$. A soma dos coeficientes de $\boldsymbol{\gamma}_{21}, \boldsymbol{\gamma}_{22} \in \boldsymbol{\gamma}_{24} \in$ igual ao coeficiente de $\alpha_{2}\left(L_{9}\right)$. bem como a soma dos coeficientes de $\gamma_{92}$ e $\gamma_{99}$ é igual a $-L_{2}-L_{9}$ Cooficientes de $\alpha_{9}$.

Da mesma forma, somando-se os coeficientes dos parámetros $\gamma$ correspondentes a $\beta_{1}, \beta_{2}, \beta_{9}$ e $\beta_{4}$ se obterá zero como resultado.

Fazendo-se $L_{2}=1, L_{9}=0$ e $L_{2}=0, L_{9}=1$, obtém-se uma hipótese tipo IV para o fator A.

$$
H_{0}^{(\theta)}:\left\{\begin{array}{l}
\alpha_{1} \quad-\alpha_{9}+\gamma_{19}-\gamma_{99}=0 \\
\alpha_{2}-\alpha_{9}+\gamma_{22}-\gamma_{92}=0
\end{array}\right.
$$

$H_{0}^{(\theta)}$ envolve contrastes entre médias de caselas que estão na mesma coluna, começando pela última linha.

As hipótese do tipo IV não se baseiam na notação RC , elas envol vem um subconjunto dos dados onde não aparecem caselas vazias. Por isso, são menos complexas.

Em termos do Modelo $\mathrm{M}, \mathrm{Ho}^{\langle\theta\rangle}$ é dada por:

$$
H 0^{(\theta)}: \quad\left\{\begin{array}{l}
\mu_{19}=\mu_{99} \\
\mu_{22}=\mu_{92}
\end{array}\right.
$$

Esta hipótese compara linhas um com trés e dois com três. Porém, não envolve colunas um e quatro na comparação, perdendo-se grande parte da informação experi mental.

A soma de quadrados tipo IV para o fator A é obtida calculando-se: 


$$
\begin{aligned}
& S Q H_{0}^{(\theta)}=\left[\mathrm{B}^{\prime}\langle\theta\rangle^{\prime}\right]^{0}\left[\mathrm{~B}_{\langle\theta\rangle^{\prime}}\left(W^{\prime} W\right\rangle^{-1} \mathrm{~B}_{\langle\theta\rangle}\right]^{-1}\left[\mathrm{~B}_{\langle\theta\rangle^{\prime}}^{\prime} \mu^{0}\right] \\
& \text { onde } \quad B_{(\theta\rangle}^{\prime}=\left[\begin{array}{llllllrr}
0 & 1 & 0 & 0 & 0 & 0 & 0 & -1 \\
0 & 0 & 0 & 0 & 1 & 0 & -1 & 0
\end{array}\right] \\
& \mathrm{SQHo}^{(\theta)}=1968,833
\end{aligned}
$$

Para se obter as funçỡes estimáveis para o fator B,tomar a forma geral de funçơes estimáveis e igualar $L_{1}, L_{2} \in L_{9}$ a zero.

Como a hipotese tipo IV sobre colunas só considera as médias pertencentes as linhas que possuem dados na última coluna, $\boldsymbol{\mu}_{\mathbf{9 2}} \in \boldsymbol{\mu}_{\mathbf{9 9}}$ não aparecerão na hipótese. Portanto os coeficientes de $\boldsymbol{\gamma}_{92} \in \boldsymbol{\gamma}_{93}$ serão zeros.

$L_{0} e L_{10}$ serão escritos em função de $L_{5} \in L_{7}$ Ccoeficientes de $\beta_{1} \in \beta_{9}$, respectivamente.

Como o nivel um do fator. B aparece nos componentes $\gamma_{11} e \gamma_{21}$ da interação $e L_{0}=1 / K_{1} L_{5}, K_{1}$ deverá ser igual a dois $e L_{0}=1 / 2 L_{5}$.

Entretanto, o nivel trés do fator $B$ só aparece em $\gamma_{19}$ co coeficiente de $\gamma_{99} e$ igual a zeros $e$ $\mathrm{L}_{10}=\mathrm{L}_{7}$

A. Tabela 36 exibe as funçơes estimáveis obtidas. 
Tabel a 36 - Funçơes Estimáveis Tipo IV para B.

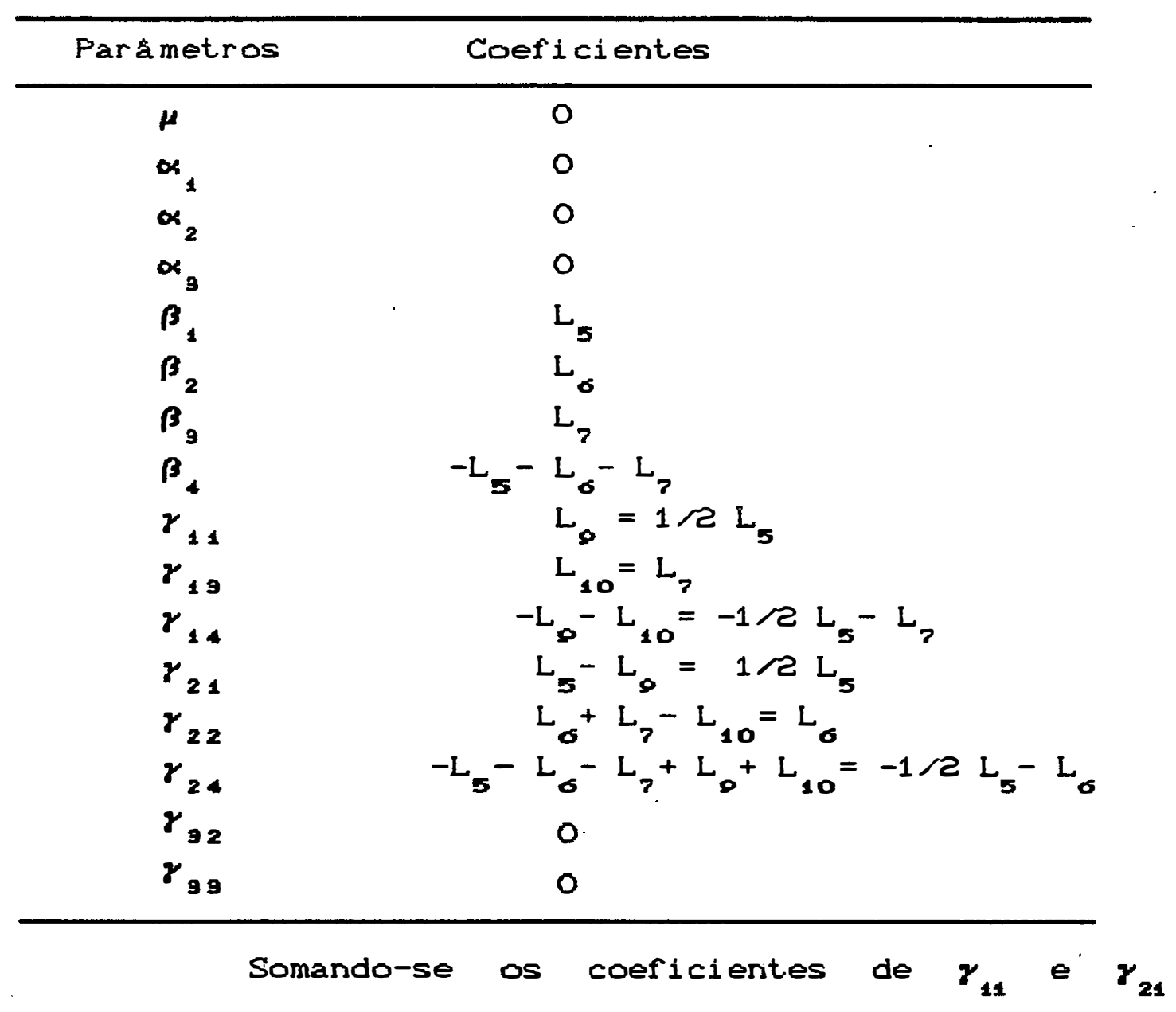

obtém-se $L_{5}$ coeficiente de $\beta_{1} 3$.

O coeficiente de $\gamma_{22} \in L_{0}$ lo mesmo de $\beta_{2}$. pois o coeficiente de $\boldsymbol{\gamma}_{92}$ e zero. Da mesma forma para $\boldsymbol{\gamma}_{19}$. Somando-se os coeficientes de $\gamma_{14} e \gamma_{24}$ obtém-se $-L_{5}-L_{6}-L_{7}$ coeficiente de $\beta_{4}^{3}$.

A soma dos coeficinetes dos parametros $\gamma$ correspondentes a $\alpha_{1}, \alpha_{2}$ e $\alpha_{9}$ devera ser zero.

Tomando-se $L_{5}=1, L_{0}=0, L_{7}=0 ; L_{5}=0$. $L_{\sigma}=1, L_{7}=0 ; L_{5}=0, L_{\sigma}=0 \in L_{7}=1$.

obtém-se uma hipótese tipo IV para o fator B (doses?. 
$148^{\circ}$.

$$
H_{0}^{(\theta)}:\left\{\begin{array}{cc}
\beta_{1} & -\beta_{4}+1 / 2 \gamma_{11}-1 / 2 \gamma_{14}+1 / 2 \gamma_{21}-1 / 2 \gamma_{24}=0 \\
\beta_{2}-\beta_{4}+\gamma_{22}-\gamma_{24} & =0 \\
\beta_{9}-\beta_{4}+\gamma_{19}-\gamma_{14} & =0
\end{array}\right.
$$

Esta hipótese é constituida por contrastes entre médias de caselas da mesma linha, começando pela úl ti ma col una.

E de mais fácil interpretaçăo pois envolve apenas um subconjunto dos dados onde não ocorrem caselas vazias.

Em termos dos parametros do Modelo M, a hipotese fica:

$$
H o(\theta):\left\{\begin{array}{l}
\frac{\mu_{11}+\mu_{21}}{2}=\frac{\mu_{14}+\mu_{24}}{2} \\
\mu_{22}=\mu_{24} \\
\mu_{19}=\mu_{14}
\end{array}\right.
$$

A primeira linha da hipótese compara col unas um e quatro, desconsiderando linha trés.

A segunda linha da hipótese compara colunas dois e quatro, considerando somente linha dois.

A terceira linha da hipótese compara colunas tres e quatro, considerando somente os dados da linha um. Observa-se que na hipótese tipo IV é mais lógico o que está sendo testado, porém com perda de infor mação.

A soma de quadrados tipo IV para o fator B (doses) é obtida calculando-se 


$$
\begin{aligned}
& S O H_{0}^{(O)}=\left[\mathrm{B}^{\prime}(\rho)^{\mu^{0}}\right]^{\prime}\left[\mathrm{B}^{\prime}(0)^{\left(O W^{\prime}\right.} W^{-1} \mathrm{~B}_{(0)}\right]^{-1}\left[\mathrm{~B}^{\prime}(\rho)^{\prime} \mu^{0}\right] \\
& \text { onde } \quad B_{i 0)}^{\prime}=\left[\begin{array}{cccccccc}
1 / 2 & 0 & -1 / 2 & 1 / 2 & 0 & -1 / 2 & 0 & 0 \\
0 & 0 & 0 & 0 & 1 & -1 & 0 & 0 \\
0 & 1 & -1 & 0 & 0 & 0 & 0 & 0
\end{array}\right] \\
& \mathrm{SOHO}{ }^{(0)}=1494,333
\end{aligned}
$$

\section{2.7. Consequências da Alteraçăo da Ordem de Especificaçăo do Modelo ao PROC GLM}

Serão apresentadas agora, as funçชes estimáveis tipo I que sofrem alteraçăo com a ordem com que as variáveis de classificaçăo săo especificadas no modelo.

Se o modelo for especificado na ordenação $B$, A. AxB ao PROC GLM, a hipótese tipo I para o fator B será uma hipótese sobre médias ponderadas de colunas não ajustadas para linhas, representada por $R(\beta \mid \mu)$.

As funçס̃es estimaveis tipo I para o fator $B$ são obtidas fazendo-se a seguinte partição na matriz X:

$$
x_{1}=\left[\begin{array}{lllll}
1 & 1 & 0 & 0 & 0 \\
1 & 0 & 0 & 1 & 0 \\
1 & 0 & 0 & 1 & 0 \\
1 & 0 & 0 & 0 & 1 \\
1 & 1 & 0 & 0 & 0 \\
1 & 0 & 1 & 0 & 0 \\
1 & 0 & 0 & 0 & 1 \\
1 & 0 & 0 & 0 & 1 \\
1 & 0 & 1 & 0 & 0 \\
1 & 0 & 1 & 0 & 0 \\
1 & 0 & 0 & 1 & 0
\end{array}\right] \quad x_{2}=\left[\begin{array}{lll}
1 & 0 & 0 \\
1 & 0 & 0 \\
1 & 0 & 0 \\
1 & 0 & 0 \\
0 & 1 & 0 \\
0 & 1 & 0 \\
0 & 1 & 0 \\
0 & 1 & 0 \\
0 & 0 & 1 \\
0 & 0 & 1 \\
0 & 0 & 1
\end{array}\right]
$$


151.

$$
x_{3}=\left[\begin{array}{llllllll}
1 & 0 & 0 & 0 & 0 & 0 & 0 & 0 \\
0 & 1 & 0 & 0 & 0 & 0 & 0 & 0 \\
0 & 1 & 0 & 0 & 0 & 0 & 0 & 0 \\
0 & 0 & 1 & 0 & 0 & 0 & 0 & 0 \\
0 & 0 & 0 & 1 & 0 & 0 & 0 & 0 \\
0 & 0 & 0 & 0 & 1 & 0 & 0 & 0 \\
0 & 0 & 0 & 0 & 0 & 1 & 0 & 0 \\
0 & 0 & 0 & 0 & 0 & 1 & 0 & 0 \\
0 & 0 & 0 & 0 & 0 & 0 & 1 & 0 \\
0 & 0 & 0 & 0 & 0 & 0 & 1 & 0 \\
0 & 0 & 0 & 0 & 0 & 0 & 0 & 1
\end{array}\right]
$$

onde:

$X_{1} \in$ formada pelas colunas da matriz $X$ correspondentes a $\mu$ e ao fator B.

$X_{2}$ eformada pelas colunas de $X$ correspondentes ao fator $A$.

$X_{9}$ formada pela colunas de $X$ correspondentes à interação.

$$
\begin{aligned}
& G_{1}=\left[x_{1}^{0} x_{1}\left|x_{1}^{\prime} x_{2}\right| x_{1}^{\prime} x_{3}\right]= \\
& =\left[\begin{array}{llllllllllllllll}
11 & 2 & 3 & 3 & 3 & 4 & 4 & 3 & 1 & 2 & 1 & 1 & 1 & 2 & 2 & 1 \\
2 & 2 & 0 & 0 & 0 & 1 & 1 & 0 & 1 & 0 & 0 & 1 & 0 & 0 & 0 & 0 \\
3 & 0 & 3 & 0 & 0 & 0 & 1 & 2 & 0 & 0 & 0 & 0 & 1 & 0 & 2 & 0 \\
3 & 0 & 0 & 3 & 0 & 2 & 0 & 1 & 0 & 2 & 0 & 0 & 0 & 0 & 0 & 1 \\
3 & 0 & 0 & 0 & 3 & 1 & 2 & 0 & 0 & 0 & 1 & 0 & 0 & 2 & 0 & 0
\end{array}\right] \\
& G_{1}^{*}=\left(x_{1}: x_{1}\right)^{-} G_{1}= \\
& =\left[\begin{array}{ccccc|ccc|cccccccc}
1 & 0 & 0 & 0 & 1 & 1 / 3 & 2 / 3 & 0 & 0 & 0 & 1 / 3 & 0 & 0 & 2 / 3 & 0 & 0 \\
0 & 1 & 0 & 0 & -1 & 1 / 8 & -1 / 8 & 0 & 1 / 2 & 0 & -1 / 3 & 1 / 2 & 0 & -2 / 3 & 0 & 0 \\
0 & 0 & 1 & 0 & -1 & -1 / 3 & -1 / 3 & 2 / 3 & 0 & 0 & -1 / 3 & 0 & 1 / 3 & -2 / 3 & 2 / 3 & 0 \\
0 & 0 & 0 & 1 & -1 & 1 / 3 & -2 / 3 & 1 / 3 & 0 & 2 / 3 & -1 / 3 & 0 & 0 & -2 / 3 & 0 & 1 / 3 \\
0 & 0 & 0 & 0 & 0 & 0 & 0 & 0 & 0 & 0 & 0 & 0 & 0 & 0 & 0 & 0
\end{array}\right]_{L_{3}}^{L_{1}}
\end{aligned}
$$

Atraves de $G_{1}^{*} \theta$ obtem-se as funçశes estimáveis apresentadas na Tabela 37 Ctomando-se a primeira linha de $G_{1}^{*}$ 
152.

como zeros.

Tabela 37 - Funçỡes Estimáveis Tipo I para B. (Ordem B, A, AxB)

\begin{tabular}{cc}
\hline Parametros & Coeficientes \\
\hline$\beta_{1}$ & 0 \\
$\beta_{1}$ & $\mathrm{~L}_{2}$ \\
$\beta_{2}$ & $\mathrm{~L}_{9}$ \\
$\beta_{9}$ & $\mathrm{~L}_{4}$ \\
$\beta_{4}$ & $-\mathrm{L}_{2}-\mathrm{L}_{9}-\mathrm{L}_{4}$ \\
$\alpha_{1}$ & $1 / 6 \mathrm{~L}_{2}-1 / 3 \mathrm{~L}_{9}+1 / 3 \mathrm{~L}_{4}$ \\
$\alpha_{2}$ & $-1 / 6 \mathrm{~L}_{2}-1 / 3 \mathrm{~L}_{9}-2 / 3 \mathrm{~L}_{4}$ \\
$\alpha_{9}$ & $2 / 3 \mathrm{~L}_{9}+1 / 3 \mathrm{~L}_{4}$ \\
$\gamma_{11}$ & $1 / 2 \mathrm{~L}_{2}$ \\
$\gamma_{13}$ & $2 / 3 \mathrm{~L}_{4}$ \\
$\gamma_{14}$ & $-1 / 3 \mathrm{~L}_{2}-1 / 3 \mathrm{~L}_{9}-1 / 3 \mathrm{~L}_{4}$ \\
$\gamma_{21}$ & $1 / 2 \mathrm{~L}_{2}$ \\
$\gamma_{22}$ & $1 / 3 \mathrm{~L}_{9}$ \\
$\gamma_{24}$ & $-2 / 3 \mathrm{~L}_{2}-2 / 3 \mathrm{~L}_{9}-2 / 3 \mathrm{~L}_{4}$ \\
$\gamma_{92}$ & $2 / 3 \mathrm{~L}_{9}$ \\
$\gamma_{99}$ & $1 / 3 \mathrm{~L}_{4}$ \\
\hline
\end{tabular}

Fazendo-se $L_{2}=1, L_{9}=0, L_{4}=0 ; L_{2}=0$, $L_{3}=1, L_{4}=0 ; L_{2}=0, L_{3}=0, L_{4}=1$

Obtém-se:

$H o$ (4) $\left\{\begin{array}{l}\beta_{1}-\beta_{4}+1 / 6 \alpha_{1}-1 / 6 \alpha_{2}+1 / 2 \gamma_{14}-1 / 3 \gamma_{14}+1 / 2 \gamma_{21}-2 / 3 \gamma_{24}=0 \\ \beta_{2}-\beta_{4}-1 / 3 \alpha_{1}-1 / 3 \alpha_{2}+2 / 3 \alpha_{9}-1 / 3 \gamma_{14}+1 / 3 \gamma_{22}-2 / 3 \gamma_{24}+2 / 3 \gamma_{92}=0 \\ \beta_{2}-\beta_{4}+1 / 3 \alpha_{1}-2 / 3 \alpha_{2}+1 / 3 \alpha_{9}+2 / 3 \gamma_{19}-1 / 3 \gamma_{14}-2 / 3 \gamma_{24}+1 / 3 \gamma_{99}=0\end{array}\right.$ 
153.

ou, em termos do Modelo M:

$$
H_{0}(4):\left\{\begin{array}{l}
1 / 2 \mu_{11}-1 / 3 \mu_{14}+1 / 2 \mu_{21}-2 / 3 \mu_{24}=0 \\
-1 / 3 \mu_{14}+1 / 3 \mu_{22}-2 / 3 \mu_{24}+2 / 3 \mu_{92}=0 \\
2 / 3 \mu_{19}-1 / 3 \mu_{14}-2 / 3 \mu_{24}+1 / 3 \mu_{99}=0
\end{array}\right.
$$

A hipótese tipo I para o fator A, quando este e especificado após o fator $B$ no modelo, é uma hipótese sobre linhas ajustadas para colunas como a hipotese tipo II para A obtida pela ordenação A, B, AxB, apresentada na Tabela 29 .

E representada por $\mathrm{R}(\alpha \mid \mu, \beta)$. 
Tabela 38 - Análise de Variancia para os Dados da Tabela 23 Através do Modelo com Dois Fatores e Interação, para Ordenação $A, B, A x B$.

TIPO I

\begin{tabular}{|c|c|c|c|c|}
\hline $\begin{array}{c}\text { Variaçত̃es } \\
\text { Consideradas }\end{array}$ & $\begin{array}{l}\text { Graus de } \\
\text { Liberdade }\end{array}$ & $\begin{array}{l}\text { Hi pot eses } \\
\text { Testadas }\end{array}$ & RC 3 & $\begin{array}{l}\text { Soma de } \\
\text { Quadrados }\end{array}$ \\
\hline$A$ & 2 & $\mathrm{Ho}^{(1)}$ & $R C \propto \mid \mu)$ & 1535,545 \\
\hline B & 3 & $\mathrm{Ho}_{0}\langle 5\rangle$ & $R(\beta \mid \mu, \infty)$ & 1815,250 \\
\hline$A \times B$ & 2 & $\mathrm{Ho}_{0}\langle 7\rangle$ & $R C \gamma \mid \mu, \infty, \beta$ & 24,750 \\
\hline \multicolumn{5}{|c|}{ TIPO II } \\
\hline$A$ & 2 & $\mathrm{Ho}^{(2)}$ & $\mathrm{RC} \propto \mid \mu, \beta)$ & 2238,083 \\
\hline B & 3 & $\mathrm{Ho}^{(5)}$ & $\operatorname{RC} \beta \mid \mu, \alpha)$ & 1815,250 \\
\hline$A \times B$ & 2 & $\mathrm{Ho}^{(7)}$ & $\mathrm{RC} \boldsymbol{\gamma} \mid \boldsymbol{\mu}, \boldsymbol{\alpha}, \beta)$ & 24,750 \\
\hline
\end{tabular}

TI PO III

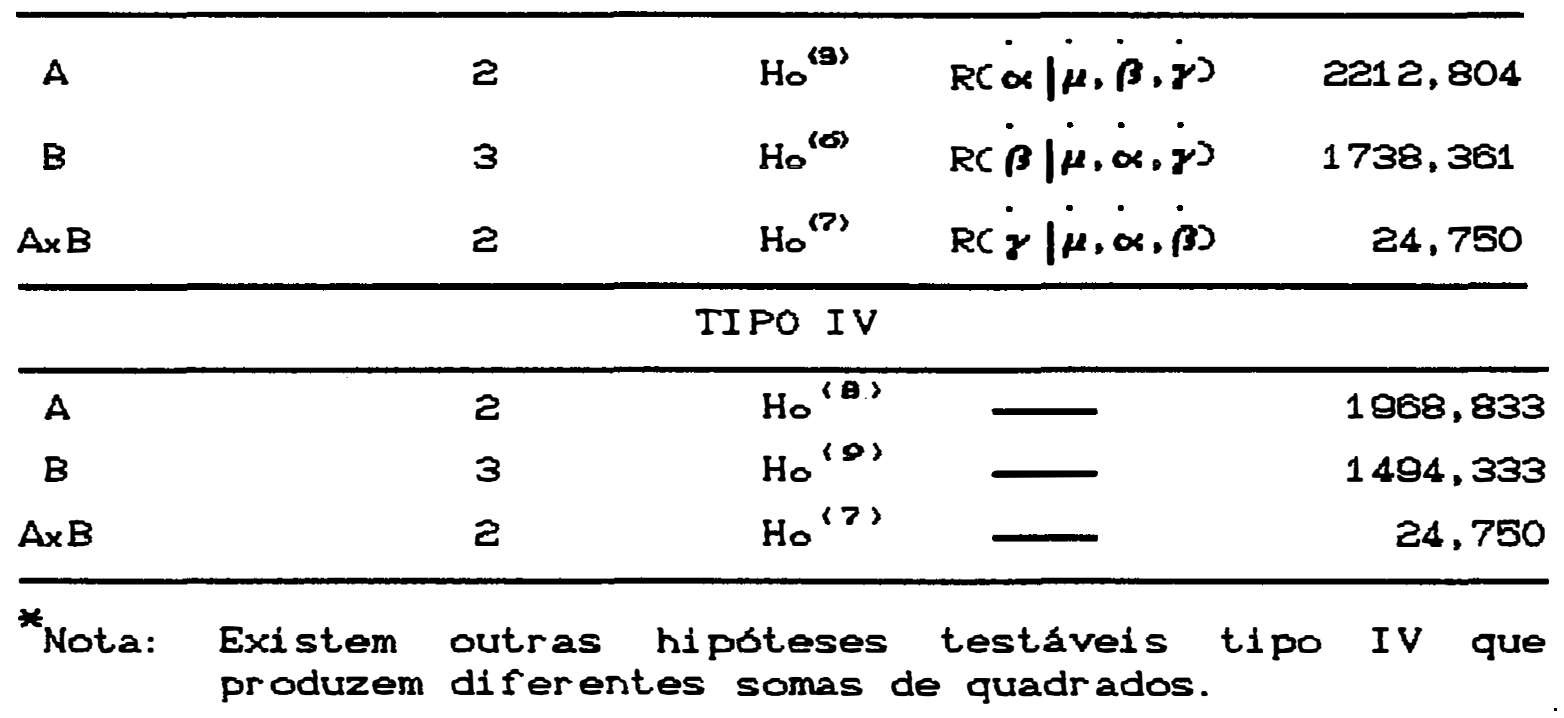


155.

Tabel a 39 - Análise de Variancia para os Dados da Tabela 23 Através do Modelo com dois Fatores e Interação, para Ordenação $B, A$, AxB.

TI PO I

\begin{tabular}{lccccr}
\hline $\begin{array}{c}\text { Variações } \\
\text { Consideradas }\end{array}$ & $\begin{array}{c}\text { Graus de } \\
\text { Liberdade }\end{array}$ & $\begin{array}{c}\text { Hipóteses } \\
\text { Testadas }\end{array}$ & $R(2)$ & $\begin{array}{r}\text { Soma de } \\
\text { Quadrados }\end{array}$ \\
\hline B & 3 & $H_{0}^{(4)}$ & $R(\beta \mid \mu)$ & 1112,712 \\
$A$ & 2 & $H_{0}^{(2)}$ & $R(\alpha \mid \mu, \beta)$ & 2238,083 \\
$A \times B$ & 2 & $H_{0}^{(7)}$ & $R(\gamma \mid \mu, \alpha, \beta)$ & 24,750 \\
\hline
\end{tabular}

TI.PO II

\begin{tabular}{llllr}
\hline$B$ & 3 & $H_{0}^{(5)}$ & $R(\beta \mid \mu, \alpha)$ & 1815,250 \\
$A$ & 2 & $H_{0}^{(2)}$ & $R(\alpha \mid \mu, \beta)$ & 2238,083 \\
$A \times B$ & 2 & $H_{0}^{(2)}$ & $R(\gamma \mid \mu, \alpha, \beta)$ & 24,750 \\
\hline
\end{tabular}

TIPO III

\begin{tabular}{lllll}
\hline$B$ & 3 & $H_{0}^{(0)}$ & $R(\dot{\beta} \mid \dot{\mu}, \dot{\alpha}, \dot{\gamma})$ & 1738,361 \\
$A$ & 2 & $H_{0}^{(9)}$ & $R(\dot{\alpha} \mid \dot{\mu}, \dot{\beta}, \dot{\gamma})$ & 2212,804 \\
$A \times B$ & 2 & $H_{0}^{(7)}$ & $R(\dot{\gamma} \mid \dot{\mu}, \dot{\alpha}, \dot{\beta})$ & 24,750 \\
\hline
\end{tabular}

TI PO IV

\begin{tabular}{rlllr}
\hline$B$ & 3 & $H_{0}^{(0)}$ & $-1494,333$ \\
$A$ & 2 & $H_{0}^{(\theta)}$ & - & 1968,833 \\
$A \times B$ & 2 & $H_{0}^{(2)}$ & - & 24,750 \\
\hline
\end{tabular}

*Nota: Existem outras hipoteses testáveis tipo IV que produzem diferentes somas de quadrados. 
156.

\section{CONCLUSÕES}

Com relação aos quatro tipos de somas de quadrados fornecidas pelo sAS quando se trata de dados desbalanceados com caselas vazias e considerando-se um modelo de classificação dupla cruzada, pode-se concluir que: - Quando se escreve as hipóteses testadas pelos quatro tipos de somas de quadrados em termos do modelo superparametrizado, todos os tipos de hipóteses envolvem os parámetros referentes à interação, não se podendo testar os efeitos principais isoladamente. Portanto, somente a hipótese sobre a interação é livre de parametros sem interesse. Porém aqui, como já comentado, não se pode testar se todas as interaçơes são nulas e portanto, só se pode abandonar o modelo com interação baseado no conheci mento do pesquisador.

- A soma de quadrados tipo I para efeitos principais, além de envolver médias ponderadas, apresentando coeficientes confusos, envolve os parámetros da interação e os parâmetros do segundo efeito principal. Nă apresentam aparente interesse e mesmo em termos dos parámetros do modelo $\mathrm{M}$ são 
157.

dificeis de interpretar.

- A soma de quadrados tipo II apesar de testar hipóteses de efeitos principais ajustados, sempre contém combinaçơes lineares dos parámetros relativos à interação e coeficientes pouco usuais.

- A soma de quadrados tipo III testa uma hipótese de interesse no exemplo com uma única casela vazia, para o fator A que só possui dois níveis. Porém, quando o número de caselas vazias e o número de niveis do fator aumenta, elas passam a ser complexas e fogem ao interesse do pesquisador. Apesar de serem hipóteses sobre médias não ponderadas. apresentam coeficientes dificeis de interpretar.

- A soma de quadrados tipo IV testa hipóteses bem mais simples e fáceis de interpretar. Porém com perda de informação, pois só é considerado uma parte dos dados com os quais elas podem trabalhar como se fossem dados bal anceados. - Em termos do modelo $M$ algumas hipoteses se tornam mais compreensiveis e além disso, uma alternativa para o impasse da análise de dados desbal anceados citada por muitos autores e trabalhar-se com contrastes de interesse entre medias de caselas que contém dados.

Portanto, é importante salientar que as hipóteses, do tipo

$$
H_{0}: \alpha_{1}=\alpha_{2}=\ldots=\alpha_{a}=0 \text { \& } H_{0}=\beta_{1}=\beta_{2}=\ldots=\beta_{b}=0 \text {. }
$$

que são de interesse do pesquisador, não poderão ser testadas em experimentos que apresentam caselas vazias e 
158.

inter ação.

Conhecendo os mecanismos que o SAS utiliza para compor seus quatro tipos de somas de quadrados e construindo suas funçỡes estimáveis o pesquisador terá condiçơes de fazer sua opção e sem dúvida, realizar sua análise de forma mais adequada e conciente. 
159.

\section{REFERÊNCIAS BIBLIOGRÁFICAS}

BURDICK, D.S. \& HERR, D. G. Counterexamples in unbal anced two way analysis of variance. Commm, Statist. Theor. Meth., g(2): 231-241, 1980.

FREUND, R.J. The case of missing cell. The American Statistician, 34(2):94-98, 1980.

GOODNIGHT, J.H. Tests of Hipothesis in Fixed Effects Linear Models. Commmo Statist, Theor. Meth., g(2) : $167-180,1980$.

HENDERSON, C.R. Estimation of variance and covariance components. Biometrics, 9: 226-252, 1953.

HOCKING, R.R.; SPEED, F.M.; COLEMAN, A.T. Hypothesis to be tested with unbalanced data. Commmo Statist. Theor. Meth, g(2): 117-130, 1980 .

IEMMA, A.F. Testes de Hipóteses em Modelos Lineares com Amostras Desequil ibradas. Belgique, Fac. Sci. Agron. Gembl oux, 1991. $101 \mathrm{p}$.

IEMMA, A. F. Anslisis de Variama de Experimentos con Cel das Vácias, Córdoba, Argentina, o p publicaçశes, 1993. $102 p$.

IEMMA, A.F. Model os Limeares: Uma introduçăo para Profissionais da Pesquisa Agropecuaria. Imprensa Oficial Est. Paraná, Londrina, 1987. 236p. 
160.

IEMMA, A.F. ; PALM, R. ; CLAUSTRI AUX, J.J. Sobre a Construção de Projetores Ortogonais. Rev, Mat. Estat,, São Paulo, $11: 133-142,1993$.

MILLIKEN, G.A. \& JOHNSON, D.E. Anal ysis od Messy Data. New York, Van Nostrand Reinhold Company, 1984, 437 p.

OVERALL, J.E. \& SPIEGEL, D.K. Concerning least squares analysis of experimental data. Psych. Bull, $72(5)$ : $311-322,1969$.

SAS User's Guide: Statistics version. 6 edition. Cary, SAS Institute, 1990 . 846p.

SEARLE, S.R. Limear Models. New York, Wiley, 1971. 532p.

SEARLE, S.R. Arbitrary Hipotheses in Linear Models With Unbalanced Data. Comun, Statist, Theor. Meth. g(2): $181-200,1880$.

SEARLE. S.R. Linear Models for Unbalanced Data. New York, Wiley, 1987. 536p.

SPEED, F.M. \& HOCKING, R.R. The Use of the RC J Notation with Unbalanced Data. The American Statistician, $30(1): 30-33,1976$.

SPEED, F.M. ; HOCKING, R.R. ; HACKNEY, O.P. Methods of Analysis of Linear Models With Unbalanced Data. Jour. Amer. Statist, Assoc., 73: 105-112, 1978.

URQUHART, N.S. \& WEEKS, D. L. Linear Models in Messy Data: Some Problems and Alternatives. Biometrics, 34: 696-705, 1978.

YATES, F. The analysis of multiple classifications with unequal number in the diferent classes. Jour. Amer. Statist, Assoc, , 29: 51-66, 1934. 


\section{APENDI CE}


162.

PROGRAMAS SAS UTILI Zados PARA OS DOIS EXEMPLOS

Página

1. Primeiro caso: fatorial $2 \times 3$ com uma casela vazia cordem $A, B, A * B) \ldots \ldots \ldots \ldots$

2. Primeiro caso: fatorial $2 \times 3$ com uma casela vazia cordem $B, A, A * B>\ldots \ldots \ldots \ldots \ldots$

3. Segundo caso: fatorial $3 \times 4$ com quatro caselas vazias (ordem $A, B, A * B) \ldots \ldots \ldots$

4. Segundo caso: fatorial $3 \times 4$ com quatro caselas vazias cordem $B, A, A * B) \ldots \ldots \ldots$ 
163.

1. Primeiro caso: fatorial Zx3 com uma casela vazia cordem $A, B, A \neq B$ S

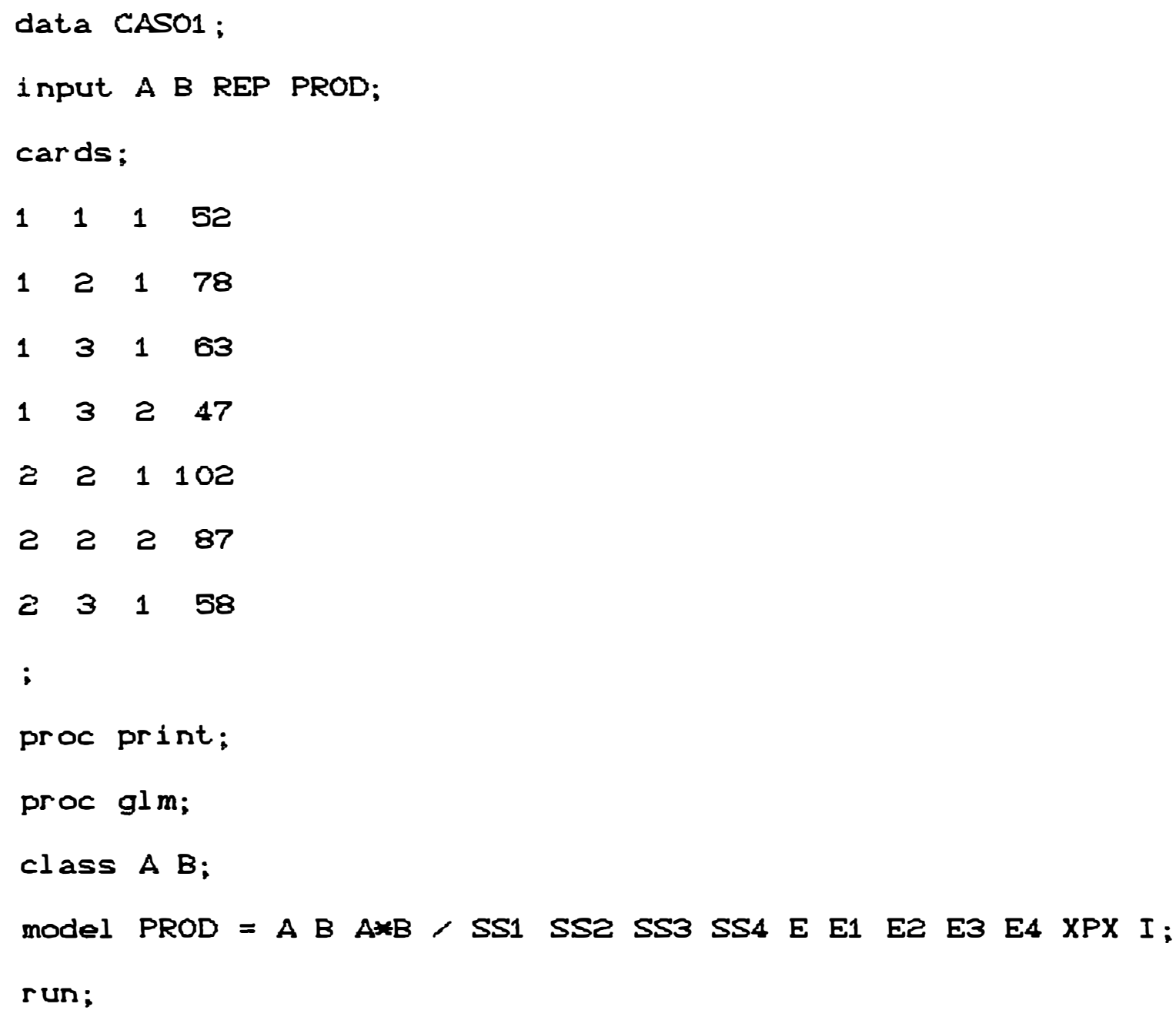


164.

2. Primeiro caso: fatorial $2 \times 3$ com uma casela vazia cordem B, A, A*BS

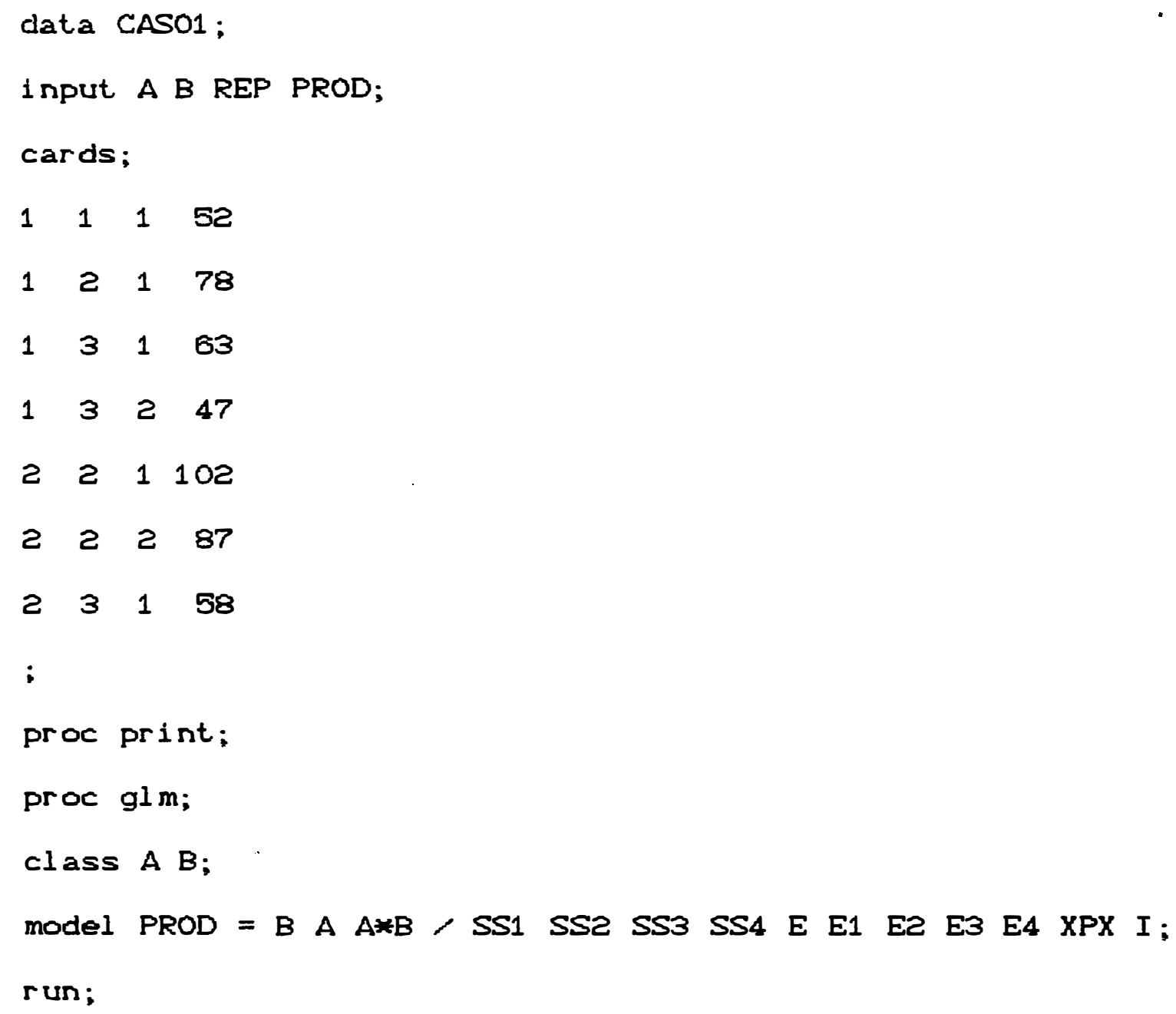


165.

\section{Segundo caso: fatorial $3 \times 4$ com quatro casel as vazias Cordem A, B, A $¥ B$ )}

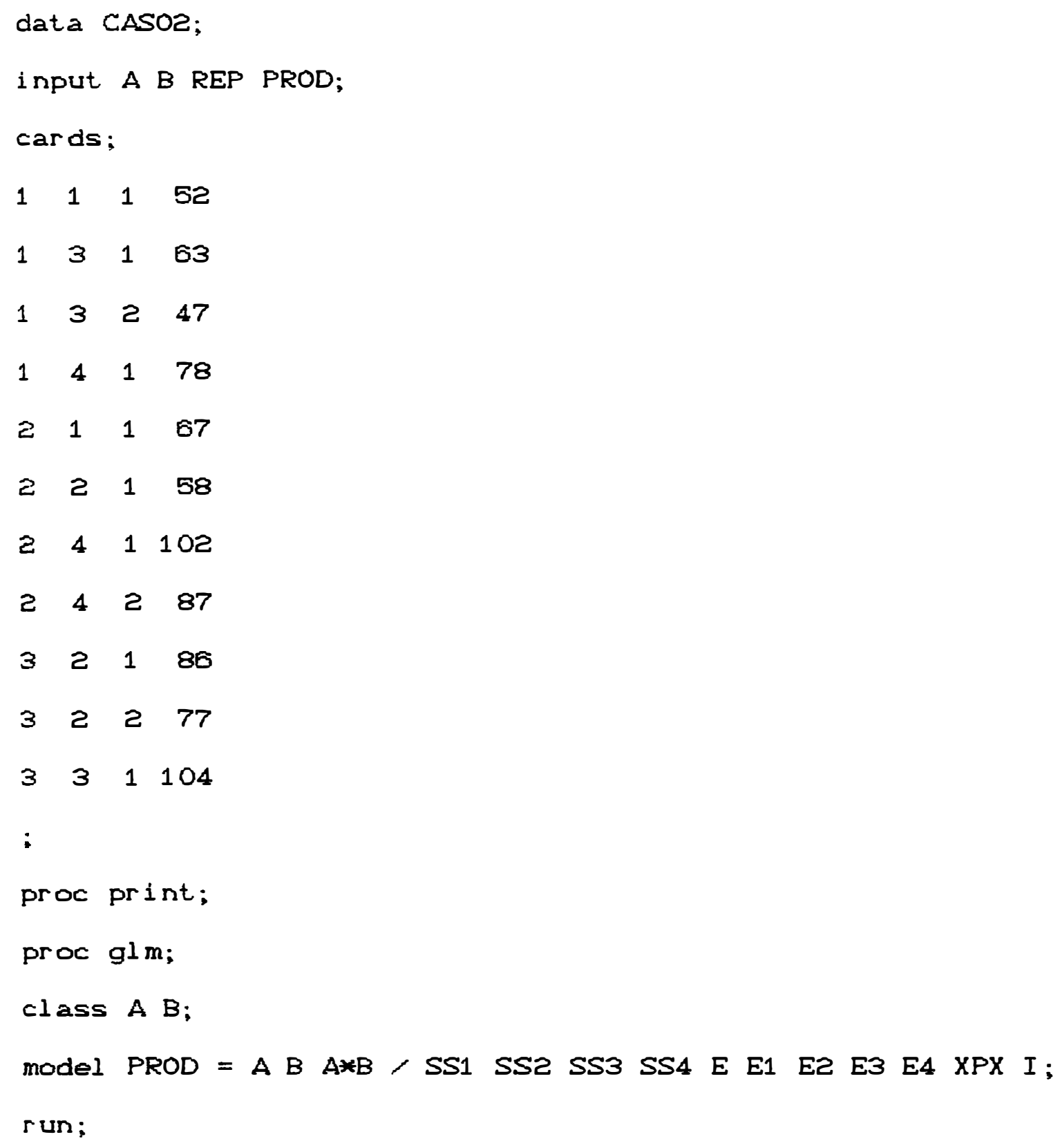


166.

\section{Segundo caso: fatorial $3 \times 4$ com quatro caselas vazias (ordem B, A, A*B)}

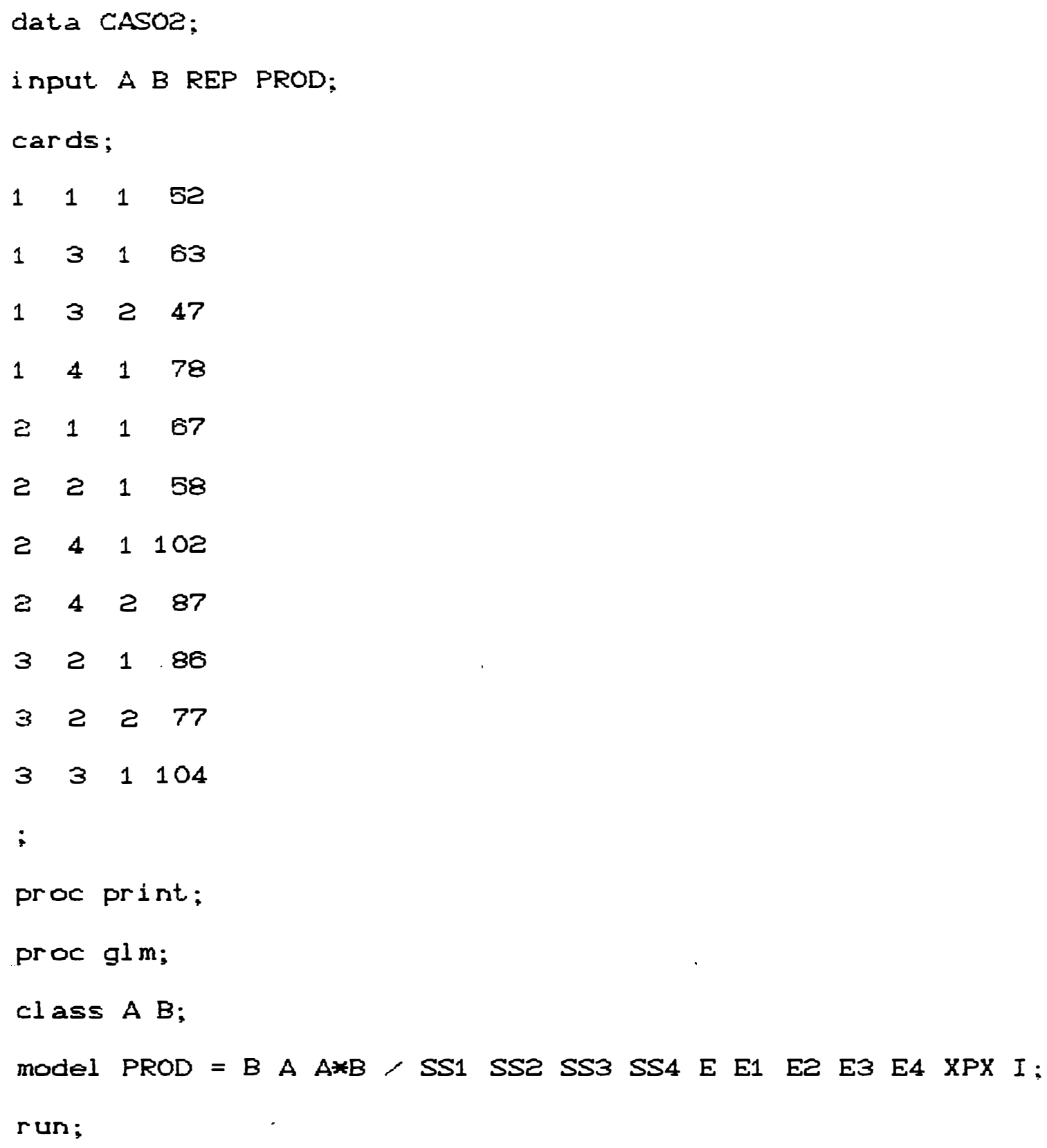

